\title{
PCGAP: Users Guide and Algorithm Description
}

E. W. Killian

J. K. Hartwell

September 2000

Idaho National Engineering and Environmental Laboratory Bechtel BWXT Idaho, LLC 


\title{
PCGAP: Users Guide and Algorithm Description
}

\author{
E. Wayne Killian \\ Jack K. Hartwell \\ September 2000 \\ Idaho National Engineering and Environmental Laboratory \\ Nuclear and Radiological Sciences Department \\ Idaho Falls, Idaho 83415
}

Prepared for the

U.S. Department of Energy

Assistant Secretary for Environmental Management

Under DOE Idaho Operations Office

Contract DE-AC07-99ID13727 


\begin{abstract}
PCGAP is a software package, which was written to provide gamma-ray pulse height spectrum analysis on a personal computer platform using the Windows NT operating system. It is a collection of programs, which provide a wide range of operability extending from the single user environment to the general purpose counting room. Included in the package are necessary data structures and libraries used to compute quantitative radionuclide concentrations. PCGAP also contains techniques to decontaminate photopeak interferences; determine activity concentrations for radionuclides independent of their associated spectral photopeak size; and three methods to determine the energy scale of a spectrum.

PCGAP performs a non-linear least squares fitting of a Gaussian function to spectral photopeaks. The fitting algorithm can fit up to five concurrent photopeaks using a common spectral background. The package includes a program that will automatically locate spectral photopeaks, fit them to a Gaussian function, identify the radionuclides associated with the found photopeaks, and compute a net activity concentration, and for an $8 \mathrm{~K}$ spectrum do it in less than 30 seconds on a reasonably configured PC.

PCGAP also includes a program which allows the operator to manually (using mouse clicks) identify photopeak locations, fit limits and background positions, initiate a fit to the photopeaks and then display the resulting Gaussian function forms. This interactive display function is particularly useful to resolve or properly fit photopeaks with complex adjacent structure and for photopeaks that are near the limit of detection.
\end{abstract}




\section{Acknowledgements}

The authors would like to thank the following colleagues for their help in making PCGAP possible.

- Bob Gehrke... Special thanks to Bob; he obtained the resources necessary to begin the development and provided many technical suggestions.

- Ron Murray, Larry Smith and Gene Taylor... These gentlemen work in the Radiation Measurements Laboratory at the INEEL and have been most helpful in identifying problems with the code when it is used in a counting room environment.

- John Baker... He used the GINA interactive peak fitting code extensively and helped find several bugs and limitations that have since been addressed.

- Sandy Hunter... She worked very hard in getting this document in a readable format. 


\section{CONTENTS}

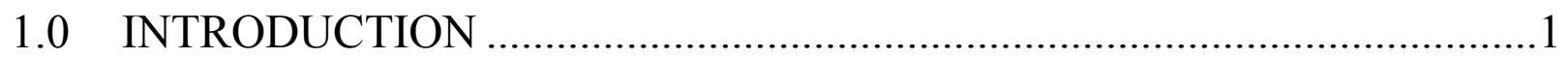

2.0 GENERAL DESIGN PHILOSOPHY AND FILE STRUCTURE.................4

2.1 Efficiency Folder...................................................................................................... 4

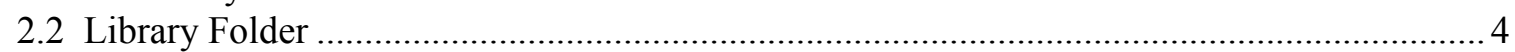

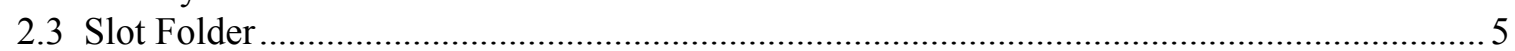

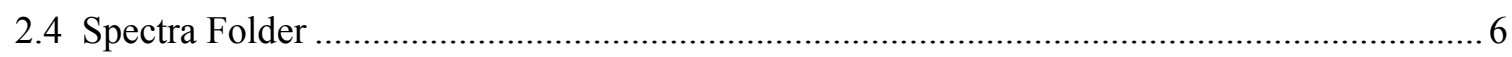

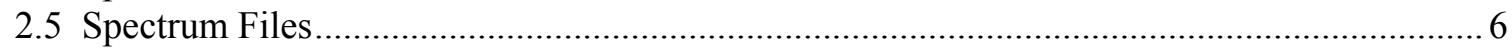

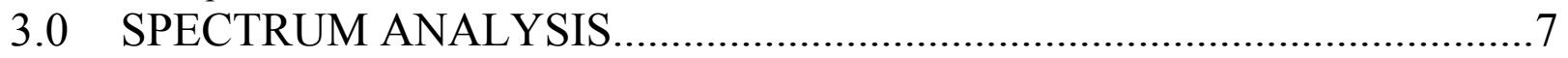

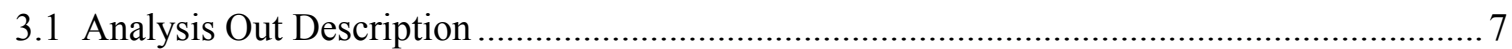

3.1.1 Photopeak Analysis Section ......................................................................................... 7

3.1.2 Nuclide Summary Section of the Analysis Output............................................................. 10

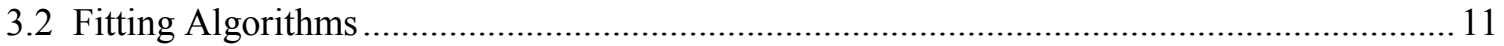

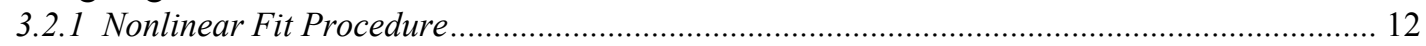

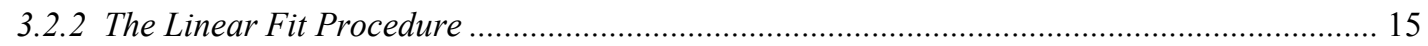

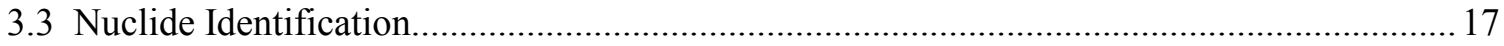

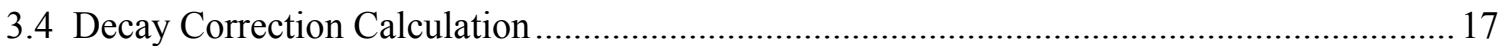

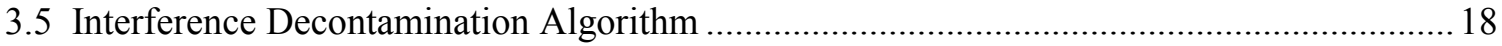

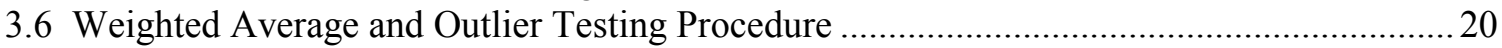

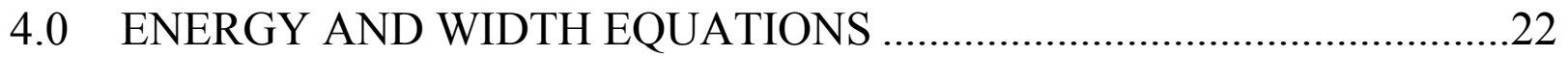

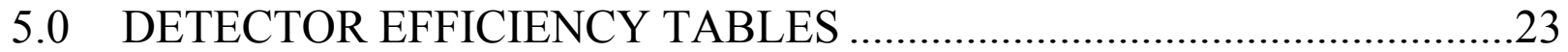

6.0 MISCELLANEOUS UTILITY PROGRAMS .........................................24

6.1 Generation of Efficiency Tables ....................................................................................24

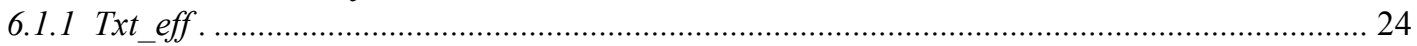

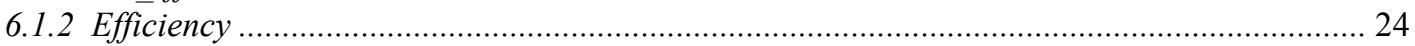

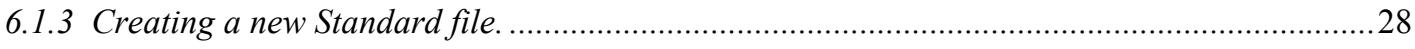

6.2 Create a Nuclide Library ............................................................................... 31

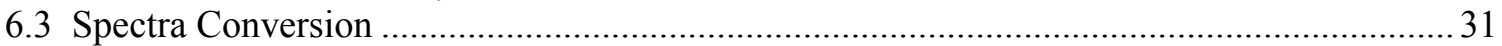

6.4 Required Library Files and Printing Output Listings............................................................ 32

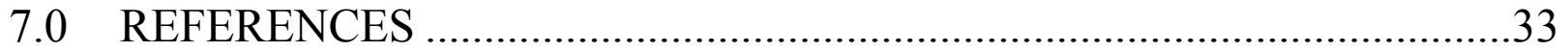

APPENDIX A - SPECTRAL PARAMETER BLOCK STRUCTURE AND

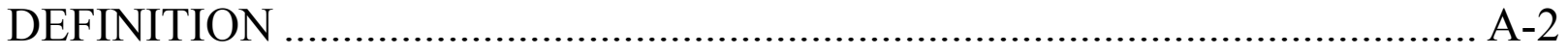

APPENDIX B - FLAG VALUES PRINTED ON PCGAP OUTPUT ...................

APPENDIX C - NUCLIDE LIBRARY ASCII INPUT FORMAT ........................2 

APPENDIX D - USERS GUIDE FOR THE INTERACTIVE PEAK FITTING

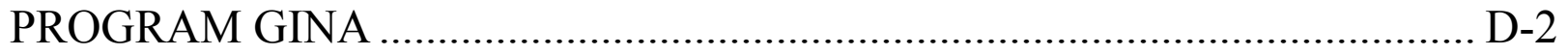

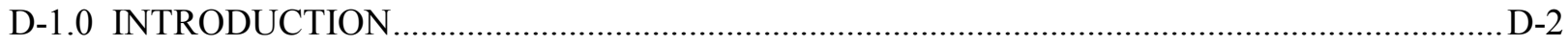

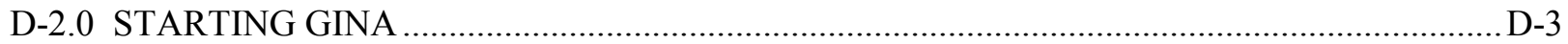

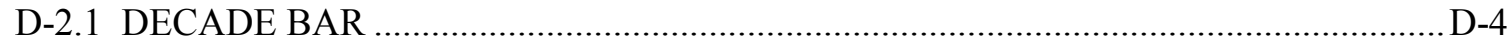

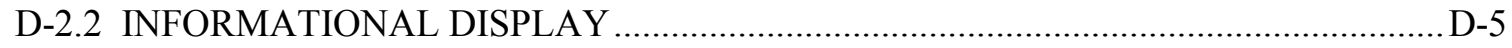

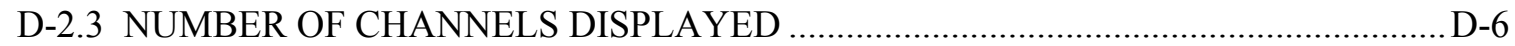

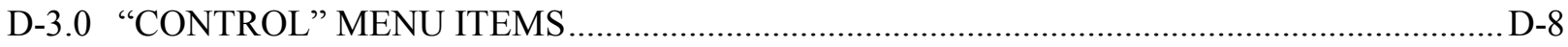
D-3.1 MARKING A PEAK FOR A FIT …........................................................................... D-9

\section{APPENDIX E - USERS GUIDE FOR THE SPECTRAL MANIPULATION PROGRAM PANDA …............................................................................}

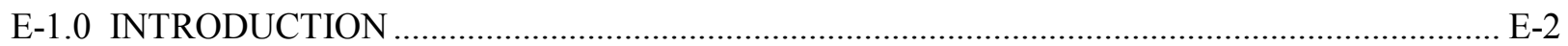

E-2.0 EXIT AND SELECT INPUT MENUS …........................................................................... E-3

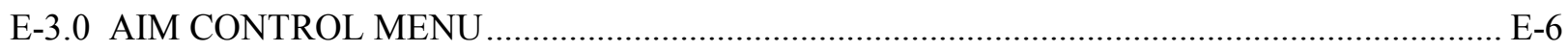

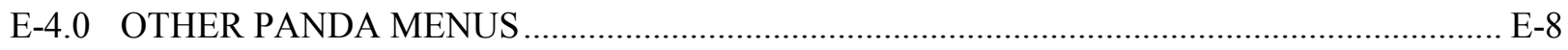

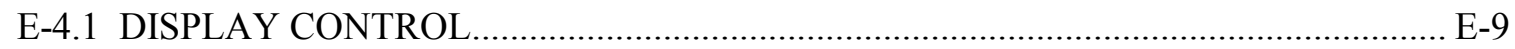

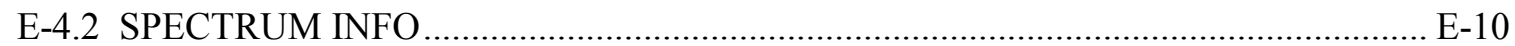

E-4.3 ANALYSIS FUNCTIONS MENU …............................................................................ 


\section{FIGURES}

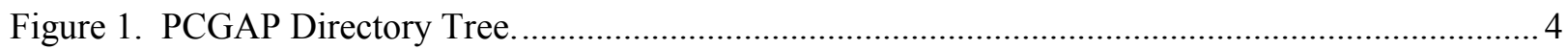

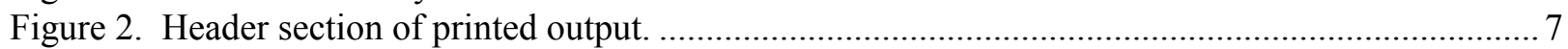

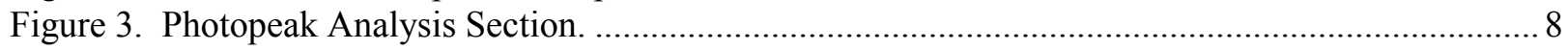

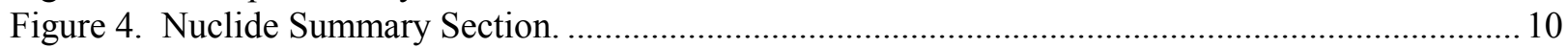

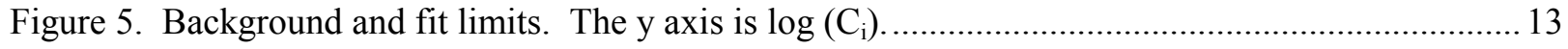

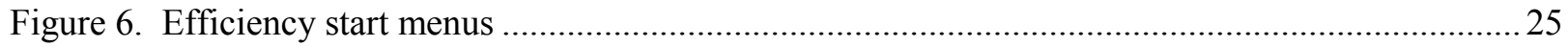

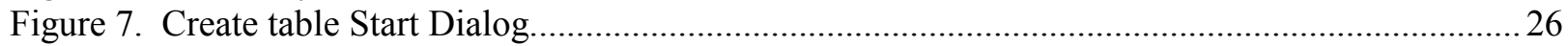

Figure 8. Generated efficiency curve

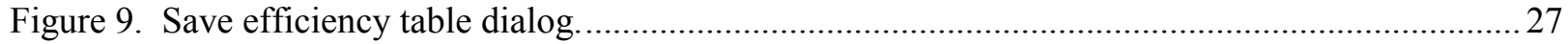

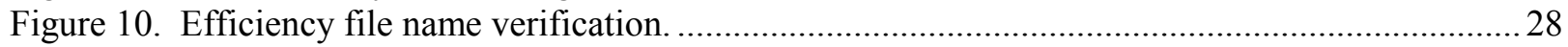

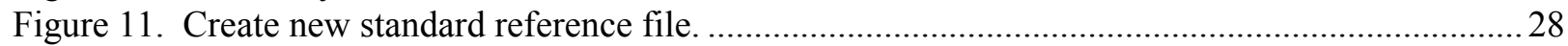

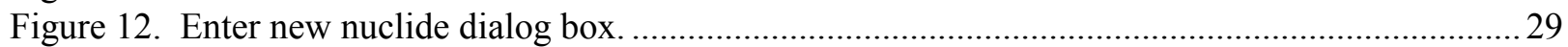

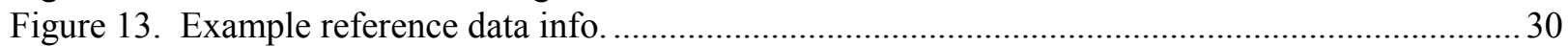

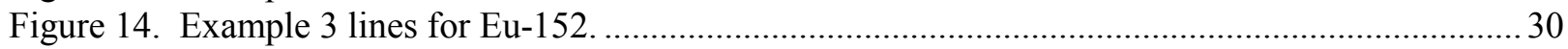

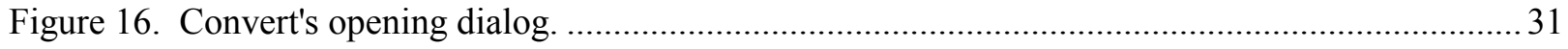

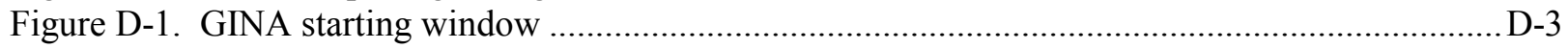

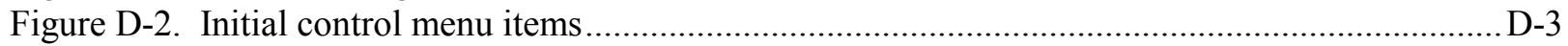

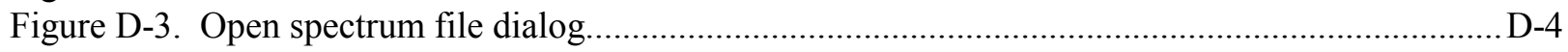

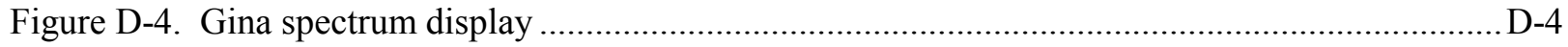

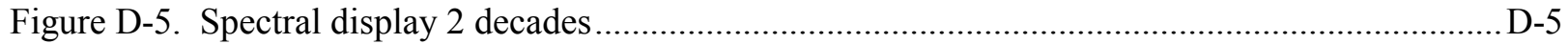

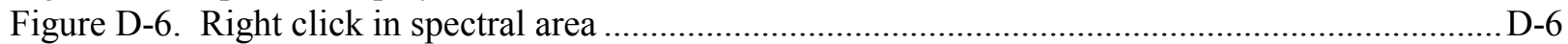

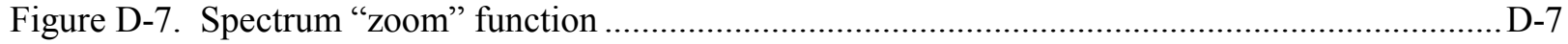

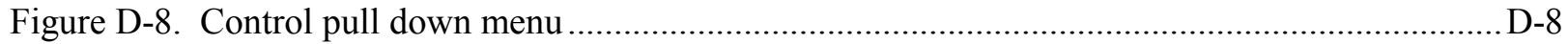

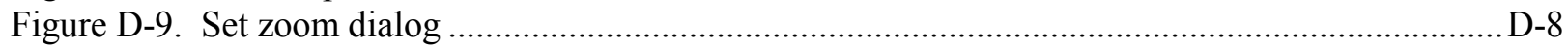

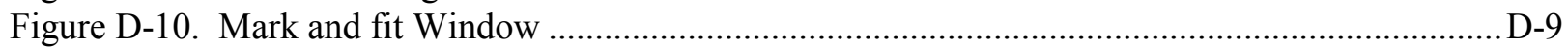

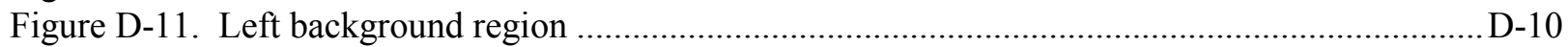

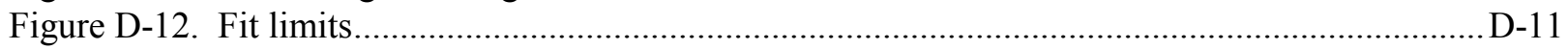

Figure D-13. Multiple fit with Centroids marked ...................................................................... D-12

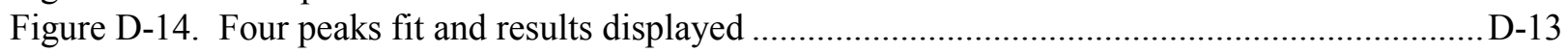

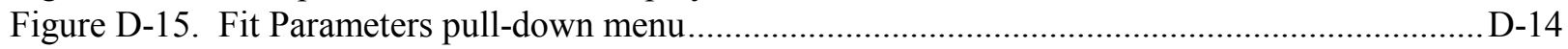

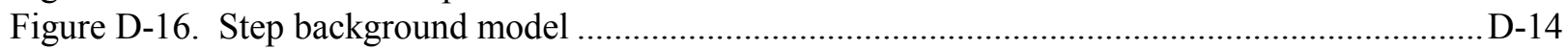

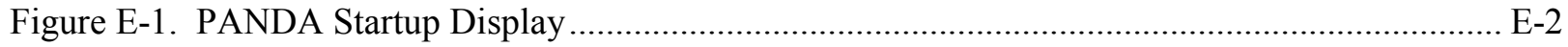

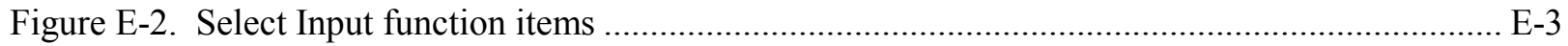

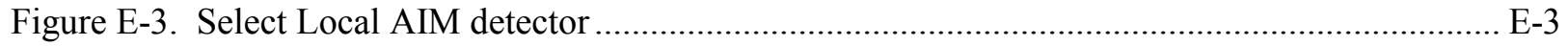

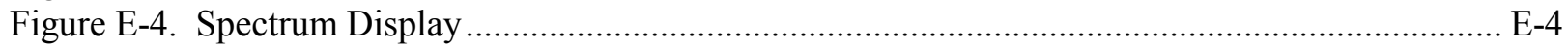

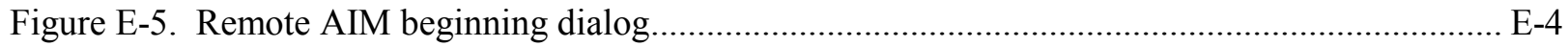

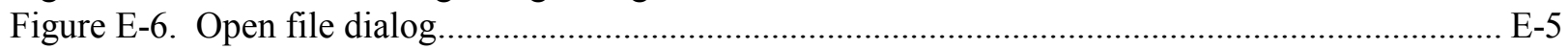

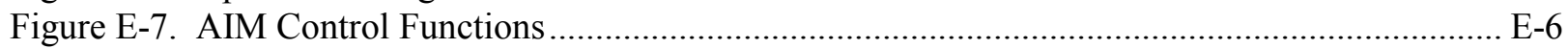

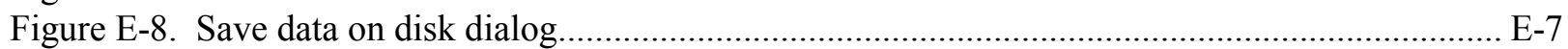

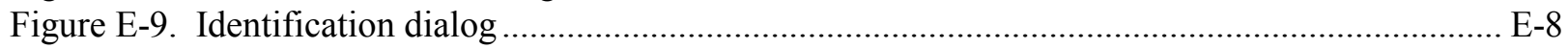

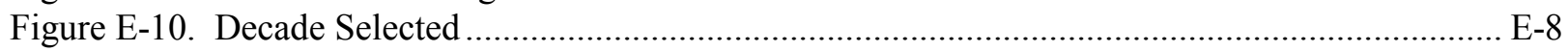

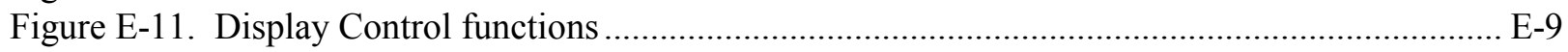

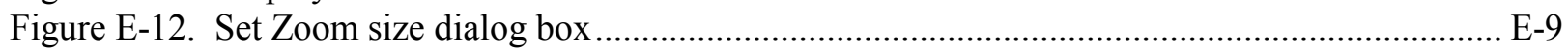

Figure E-13. Spectrum zoomed to 256 channels........................................................................... E-10

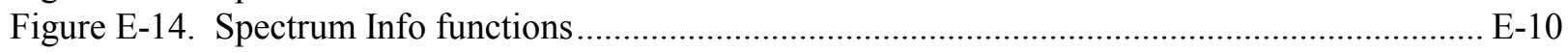

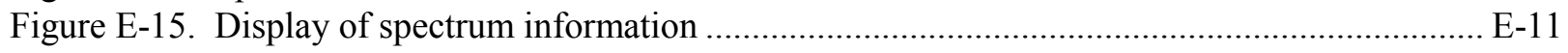

Figure E-16. Print Channel Contents dialog box ……..................................................................... E-11 


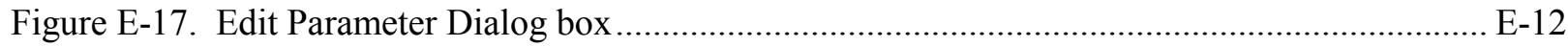

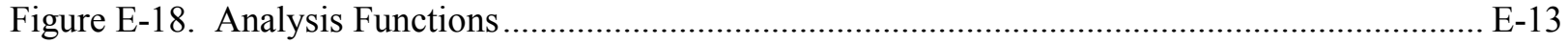

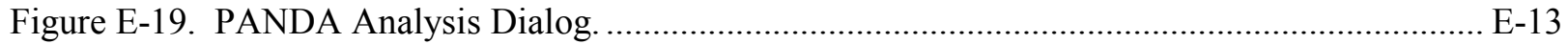

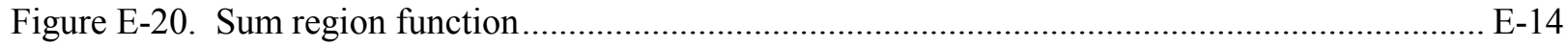

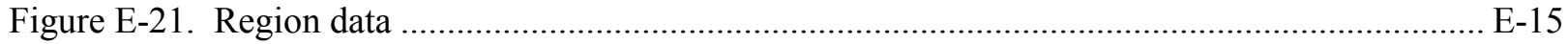

Figure E-22. Energy scale determination Dialog box .................................................................... E-16 


\section{TABLES}

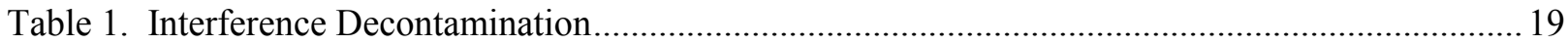

Table A-1. Spectrum Parameter Block Data Structure …................................................................. A-3

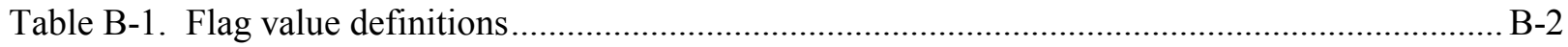




\section{PCGAP: Users Guide and Algorithm Description}

\subsection{INTRODUCTION}

PCGAP is a fully functional gamma-ray spectrum analysis package. It is a collection of programs, which provide a wide range of operability extending from the single user environment to the general purpose counting room $^{[1]}$. With suitable spectrum conversion routines, it can be used to analyze data from almost any manufacturers spectrometer system.

PCGAP can control the operation of a Canberra Acquisition Interface Module (AIM) spectrometer equipped with an INEEL developed pulse injection system for individual spectrum validation ${ }^{[2]}$.

The package includes programs for the manual analysis of spectra using displays which permit the spectroscopist to interactively define the spectral continuum and peak fitting limits, and display the resulting function fitting forms.

The following techniques and analysis algorithms are used in PCGAP.

- $\quad$ Nonlinear least squares fitting of a Gaussian function to photopeaks

The analysis of a spectrum can be performed in two ways. The first is the completely automatic method. It begins by locating the photopeaks in the spectrum. The search algorithm is a simple cross-correlation of the square root of the spectral channel contents with a zero-area square wave $^{[4]}$. The found photopeaks are fit to a Gaussian function to determine areas, centroids, widths and their uncertainties. The fitting procedure is an iterative technique, which minimizes the sum of the squares of the deviations of data from a Gaussian function form. In complex regions of a spectrum, up to five photopeaks can be fit concurrently using a common spectral background. The criteria used to determine whether or not a multiplet fit is to be performed depends on how many peak widths exist between the photopeak being fit and the next photopeak.

The second method of analysis is an interactive technique where the user utilizes the mouse pointer to identify the photopeak background regions, region of the peak(s) included in the fit and the photopeak centroids. The operator can select the use of either a linear or step photopeak background model, fixed or varying widths, with up to a maximum of 5 peaks included in one integrated fit. The derived Gaussian functions determined from the fit are displayed to the user. Also displayed are peak areas, centroids and widths. This interactive fit function can prove to be very useful in analyzing regions of the spectrum where the structure is complex or where photopeaks are weak with respect to the underlying background.

- $\quad$ Linear least-squares fitting of selected photopeaks

For those photopeaks which could not be fit by the nonlinear technique and for specific regions in the spectrum where a photopeak might be located, a linear least squares fit algorithm is used. This algorithm assumes that a photopeak is represented by a Gaussian function and a linear background. The position and width of the Gaussian are fixed during the fitting process. The Gaussian height and the coefficients of a linear spectral background line are determined from the

fit. Depending on the distribution counts of these regions of the spectrum, this algorithm can and 
will produce areas that are positive or negative. Uncensored negative activity results and their uncertainties are required for accurate estimation of certain analyses (for example, the determination of total efficient discharges from a number of periodic samples). The technique is primarily used to determine concentration values for a radionuclide regardless of its activity level.

- $\quad$ Nuclide Identification

Radionuclide identification of photopeaks present in a spectrum is done by matching the energy of the photopeak to a list of energies in a nuclide library. A maximum of 20 nuclide tags can be assigned to any one photopeak. Photopeaks are also identified as possible single and double escape peaks. Since applications can require the use of nuclide libraries containing different entries, different libraries can be constructed. A decay correction factor for each nuclide is also computed. This factor provides a correction for the decay incurred from the time the sample was collected to the start of the count and for the decay during the counting interval.

\section{- Interference Decontamination}

In a gamma-ray pulse-height spectrum, it is possible that two or more gamma-rays from different radionuclides have contributed to a single photopeak. In PCGAP, an interference decontamination algorithm determines which fraction of a single contaminated photopeak area belongs to each of the contributing nuclides, provided some or all radionuclides involved have "clean" photopeaks to provide interference information. For this algorithm, the user of the system must know what interferences could be present in the sample and, through the use of an interference library, indicate how these interferences can be resolved.

- $\quad$ Energy Scale and Photopeak Width Function Determination

The relationship between channel and energy can be established three ways in PCGAP:

1. Automatically using a pulse-injection technique ${ }^{[2]}$.

2. Automatically using one of two different calibration sources, either ${ }^{228} \mathrm{Th}$ or ${ }^{152} \mathrm{Eu}$. This is an automated process that locates a set of five photopeak lines in the calibration spectrum, and fits the peaks to determine centroids and their full widths at half maximum height (FWHM). It then performs a least squares fit of centroids and photopeak energies to determine the coefficients of a $2^{\text {nd }}$ order polynomial from which the energy of a channel can be determined. The widths and centroids are fit to a $1^{\text {st }}$ order polynomial to determine the coefficients of the FWHM versus channel function.

3. Interactively, in which a user assigns gamma-ray energy values to photopeak centroids.

- $\quad$ Concurrent Determination of Ambient Background Contribution

An improved method for determining the ambient background contribution in the analysis of gamma-ray spectra is used in PCGAP ${ }^{[3]}$. Regions in the sample spectrum containing peaks of interest are mapped to one or more background spectra to determine the contribution, if any, of ambient background activities to be subtracted from the measured sample activities. The method eliminates the need for separate detailed analyses of background spectra, and better ensures that ambient activities near the detection limit will be recognized. This is especially important for environmental samples in which small ambient backgrounds, if not properly taken into account, can cause false-positive measurements. 
The users guide for two major components of PCGAP have been attached as appendices; the interactive peak fitting program GINA, and the spectrum display and automatic spectrum analysis program, PANDA. Appendix D is the users guide for GINA and Appendix E is for PANDA. 


\subsection{GENERAL DESIGN PHILOSOPHY AND FILE STRUCTURE}

There are several different data files that are used by PCGAP to perform various functions. They include Nuclide, Required Nuclide, and Interference Decontamination libraries. Additionally, there is an efficiency table file used to perform detector efficiency calculations. The backbone of PCGAP is the spectrum data file. A spectrum file name is supplied to almost all of the PCGAP programs. A detailed description of these data files will be given in subsequent sections, but before we get to those, a general description of the file folders (directories) implemented in PCGAP is in order. The following diagram describes the PCGAP directory tree. PCGAP will always be installed as a top-level directory on a hard disk drive. The installation process creates an environmental variable "gapdisk" which will denote on which disk the top-level directory is created.

Figure 1. PCGAP Directory Tree.

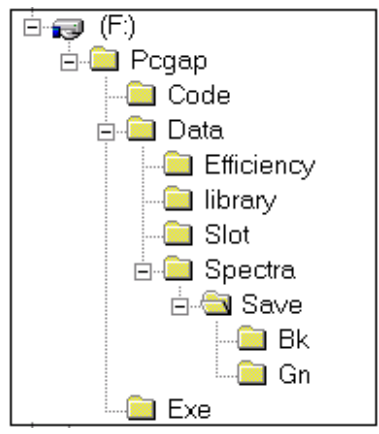

Of the three top-level sub-directories "Code", "Data", and "Exe" the typical user of PCGAP will be mostly concerned with the files located in the data folder. The Code folder contains the source files for the PCGAP programs. The Exe folder contains the program executable files. The operator can add as many sub-folders to any of the existing folders as desired. Do not delete or rename any of the folders displayed in Figure 1. All of the PCGAP programs will look in specific folders following a path the described in Figure 1 to find a specific data file. Following is a description of contents of each of the sub-folders in the Data directory.

\subsection{Efficiency Folder}

This folder contains the efficiency table files. These files describe the detector efficiency for a given source geometry. PCGAP will look in this directory for any efficiency table requested to be used during an analysis. The efficiency table files have a specific naming convention (See section 6.0).

\subsection{Library Folder}

The library folder contains three sub-folders: Inter, Nuclide and Required. These folders contain the "interference", "nuclide" and "required nuclide" libraries respectively. An interference library file describes how a photopeak that has contributions from two or more nuclides can be resolved into their individual components. This algorithm requires at least one "clean" photopeak for one of the nuclides, which is a component of the contaminated photopeak.

A nuclide library file is used to make associations with the energy of a photopeak and a radionuclide. This file contains the chemical symbol of the nuclide, atomic weight and number, half-life, 
decay branching ratio and a code number from which the relative worth of a photopeak in relation to other photopeaks for that radionuclide is determined. A fairly comprehensive library, MASTER, is provided with the PCGAP distribution.

The required library is used to determine the activity of a radionuclide at a particular energy regardless of whether the photopeak in question was found or missed by the automatic photopeak search routine. This library file contains a list of energies from which the location of required photopeaks can be determined. This option is particularly useful in providing results to regulatory agencies or in compliance with Technical Specifications because an "answer" is provided even when it is zero or negative.

\subsection{Slot Folder}

The slot folder contains at least two files, a calibration file and a spectrum data file. These two files are called a slot pair. The name of slot pair files must be the same. The file names must be a twocharacter combination such as A1 or SC. The calibration file must have the extension "CLB" and the spectrum file extension "DAT". Some examples of valid slot pairs are:

\section{A1.clb A1.dat These two files would create an A1 slot pair SC.clb SC.dat An SC slot pair}

Slot pairs or Slots are used to temporarily store information that can be accessed and used by different programs. Two PCGAP utility programs can be used to move spectral to and from the slot file. The program PANDA is used to copy data from a slot to a permanent spectrum save area (see the following section). PANDA will ask the user to supply the file name of the spectrum which will be created in the spectrum save area, a character string which describes the spectrum, the volume and volume units of the sample from which the spectrum was accumulated, and finally the efficiency table to be used when analyzing the spectrum data. The utility program SPECTRA can be used to copy spectrum files into a slot file. This function is used when a particular PCGAP spectrum manipulation/analysis program requires the input data to be located in a slot file.

The information in the calibration file for a slot pair can be written only by one of the energy scale calibration options in PANDA (see Appendix E, section E-4.3). When one of these calibration programs is executed they determine the values for the coefficients of the energy and width equations. In case an INEEL pulser-equipped AIM multi-channel analyzer is used to collect spectral data, the automated calibration routines will also determine pulser equivalent energies and store these values in the calibration block. Once the calibration parameters have been determined and stored in the calibration file for a given analyzer (e.g., A1 or Sc), subsequent transfers of spectral data from that analyzer will read the information stored in the calibration file to create a PCGAP spectrum file. To reiterate, once calibration parameters have been determined and stored in the calibration file of a slot pair, all spectrum file creations for the designated analyzer will inherit the information stored in the slot calibration file. This allows the operator to calibrate once and use those parameter values for all subsequent spectra collected by a system. The user can create as many slot pairs has he/she desires. Usual practice is to create a slot pair for every different detector system, connected to the computer. 


\subsection{Spectra Folder}

The spectra folder contains one sub-folder, SAVE, which has one or more sub-folders. Spectrum files are saved in these sub-folders. These sub-folders denoted as "Save areas" are used to save spectral data from different types of samples. For example a "Save area" could be used to store all spectra collected from a particular waste stream or area. The initial PCGAP configuration contains two "Save areas"; GN and BK. The BK save area is used to save ambient background spectral files. GN is a general "Save area" where any type spectrum can be stored. The user can create additional "Save areas"; they must be sub-folders of the SAVE directory and have two character names. Except for the BK save area, there is no restriction on which two characters can be used. Since, the analysis code examines the contents of the BK save area to determine which files to use for the ambient background correction this save area must exist.

\subsection{Spectrum Files}

Each spectrum file has a direct access format and has three different parts; a header record (512 bytes), a parameter record (512 bytes), and several records which comprise the spectral data itself. Each spectrum file is a self-contained entity in that all of the information required for a spectrum analysis is contained in the file. Following is description of the different spectrum file records.

\section{Spectrum file header record}

This 512-byte record contains character data only. In most cases when a spectrum file is created the user is asked to supply alphanumeric information which describes the spectrum. This information is stored in the last 452 bytes of header record. The first 60 bytes will contain the spectrum file name.

\section{Spectrum file parameter record}

The second record of the spectrum file is also 512 bytes long. This record contains character, integer and floating point information. The structure of this record is given in appendix B.

\section{Spectrum file spectra data records}

The pulse-height data records are 512 byte records. The data are stored as floating point numbers, consequently there are 128 channels of data per 512 byte record. The number of records per spectrum $=$ number of channels in spectrum $/ 128$.

\section{Spectrum file naming convention}

Spectrum files in PCGAP have a file extension of ".dge". A two character representation of the detector from which the data is collected is usually used as the first two characters in the file name. Some examples of valid file names: A10614000001.DGE or P10413.DGE 


\subsection{SPECTRUM ANALYSIS}

In PCGAP the program which performs the spectrum analysis is triggered by using a PCGAP utility called PANDA (see Appendix E). The analysis program will automatically locate the photopeaks in a spectrum, fit them to a Gaussian function, identify the possible radionuclides present in the sample and produce a printed output of the results. The following sections describe in detail the algorithms used to perform these functions. A description of how to use the PANDA utility can be found in Appendix E.

\subsection{Analysis Out Description}

The printed output is divided into two parts:

1. A section which describes the analysis of each of the photopeaks found /or analyzed in the spectrum. This section will be described as the "photopeak analysis" section.

2. A section, which describes the radionuclides, identified as being present in the sample. This section will be denoted as the "Nuclide" section.

A description of each of the output sections is given below. The output begins with a header section, an example of which is shown below. This header section describes the parameters used during the spectrum analysis. The meaning of most of these parameters is self explanatory. The "LIBRARIES USED" parameter indicates the names of the nuclide, required and interference libraries respectively. The section titled "PULSER CALIBRATED" would not be printed for a spectrometer which is not equipped with an INEEL pulser injection system. The "BACKGROUNDS AVERAGED" section lists the file names of the background spectrum files used during the analysis. Up to three background spectrum files can be used. If no ambient background correction is performed the space under backgrounds averaged will be blank.

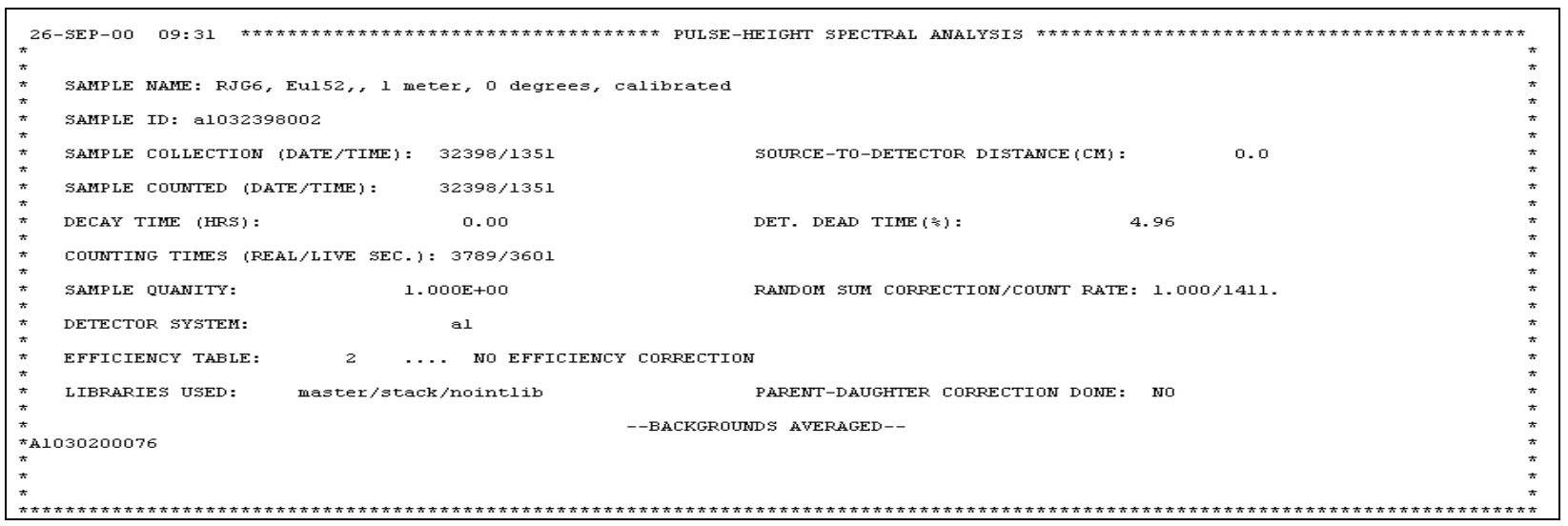

Figure 2. Header section of printed output.

\subsubsection{Photopeak Analysis Section}

The photopeak analysis section describes the analysis results of all the photopeaks in the spectrum. An example of a portion of the photopeak analysis section is given below: 


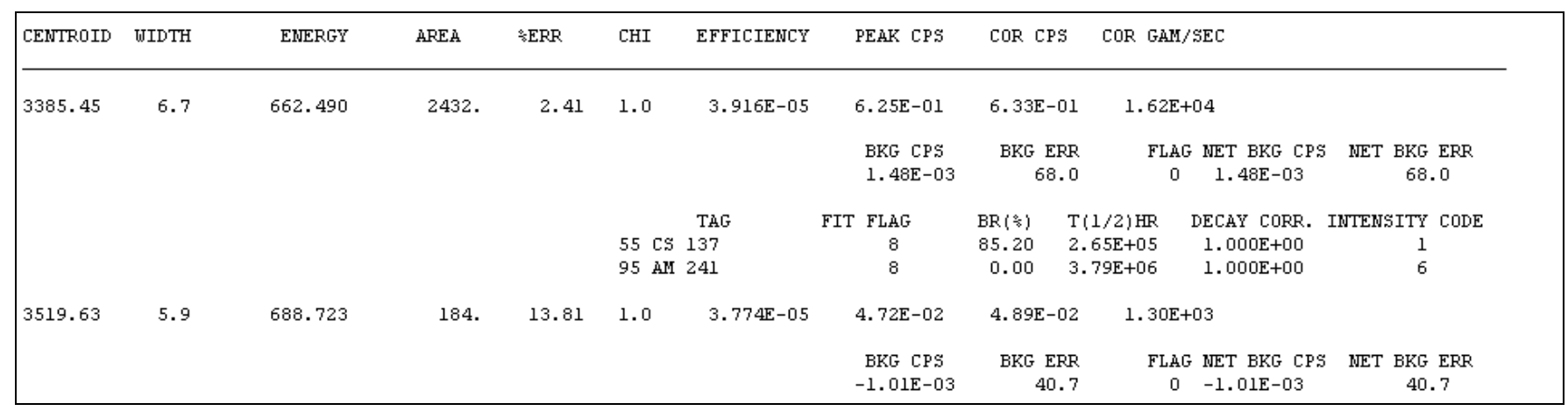

Figure 3. Photopeak Analysis Section.

Across the top of every page of out put the following heading banner will be printed:

\section{CENTROID | WIDTH $\mid$ ENERGY $\mid$ AREA | \%ERR | CHI | EFFICIENCY | PEAK CPS |COR CPS |COR GAM/SEC}

The printed values aligned with these columns are defined as follows:

CENTROID... Photopeak centroid in channels.

WIDTH... Photopeak full width at half peak maximum (FWHM) in channels.

ENERGY...Photopeak energy (keV) computed at the centroid.

AREA...The area of the photopeak.

\%ERR...The 1-sigma error in the area expressed as a percent.

CHI... The reduced chi squared value of the fit.

EFFICIENCY... The detector efficiency value at the photopeak energy.

PEAK CPS ... The area of the peak divided by the live time.

COR CPS...The peak cps (counts/second) corrected for ambient background and random summing.

COR GAM/SEC...The corrected cps divided by the efficiency value.

Following the above values for a photopeak another column of values used for the ambient background correction are printed as shown in Figure 3. The information printed in this section is particular to the concurrent background analysis technique used in the analysis ${ }^{[3]}$. Basically, regions in the sample spectrum containing peaks of interest are mapped to one or more background spectra to determine the contribution, if any, of ambient background activities to be subtracted from the measured sample activities. The method eliminates the need for separate detailed analyses of background spectra, and better insures that ambient activities near the detection limit will be recognized. Consequently, each analyzed photopeak has a computed ambient background contribution. The information in the background column contains information derived from the ambient background spectra. There will be one line of output for every background spectrum used in the analysis. In the "photopeak analysis" 
example shown above, there is just one background spectrum used; consequently, only one set of numbers is printed. The background row is titled with the following :

\section{$\underline{\text { BKG CPS } \mid \text { BKG ERR | FLAG } \mid \text { NET BKG CPS | NET BKG ERR }}$}

The numbers aligned after these column headings are defined as follows:

BKG CPS...The counts/second value in the background spectrum at the energy of the photopeak being analyzed.

BKG ERR...The percentage 1-sigma error in the "bkg cps" value.

FLAG...This value is an integer value of 0 or 4 . This value indicates whether a "bkg cps" value for a particular spectrum is used in determining the "net bkg cps" value. A flag of " 0 " indicates that the value is used to calculate the net value. A flag value of " 4 " indicates that the "bkg cps" value was not consistent with the values obtained from the other spectra (it is an outlier) and is not used to compute the net value.

NET BKG CPS... Is the inverse variance-weighted average (with outlier testing) of all "bkg cps" values.

NET BKG ERR...This is the computed error in the "net bkg cps" value.

\section{TAG $\mid$ FIT FLAG $\mid$ BR (\%)| T(1/2) HR $\mid$ DECAY CORR. $\mid$ INTENSITY CODE}

Following the ambient background information is another set of columns (see figure 3) which indicate the nuclides (extracted from the nuclide library) which might be associated with the photopeak. This nuclide identification is associated with the energy of each photopeak and includes all entries (20 max) in the library whose entries are within $1 \mathrm{keV}$ of the photopeak energy. If there are no nuclides identified with photopeak, the headings for the nuclide columns will not be printed. The nuclide information is identified with the following column headings:

The definitions of the headings are:

TAG... Is the atomic number, chemical symbol, and atomic weight of the identified nuclide.

FIT FLAG...This value is an integer value which indicates how the peak was fit. (was it part of a multiplet, ambient background corrected, etc.) Appendix B gives a detailed explanation of the values which can be assigned to a fit flag.

$\mathrm{BR}(\%) \ldots$ The branching ratio (emission probability) of the nuclide line defined as a percent.

T(1/2)HR...The half life of the nuclide expressed in hours.

DECAY CORR...Is the calculated decay correction for the identified nuclide. It is the product of two parameters. The first is the correction for the decay incurred from the time the sample was collected to the start of the count and for the decay during the counting interval. The second 
parameter is primarily for air sampling filters and provides correction for decay (if any) incurred during the sample collection.

INTENSITY CODE...This value is an integer (1-5) which indicates the relative worth of that specific photopeak in the decay of the nuclide. An intensity code $=1$ line is the "key" photopeak for a given nuclide.

\subsubsection{Nuclide Summary Section of the Analysis Output}

The first step in determining radionuclide concentration values is to group all photopeaks identified as belonging to a particular nuclide. Each nuclide line identified with a photopeak has, as part of its data structure, an intensity code which indicates the "worth" of the line compared to the other lines for this radionuclide. The smaller the value of the intensity code, the more likely it is that the line will show up in the spectrum if that particular radionuclide is present in the sample. Given a set of lines for a particular nuclide there is only one line which has an intensity code of 1 . This code indicates that this is the most prominent clean line for that nuclide. Consequently, a nuclide is probably not in the sample if the intensity code 1 line has not been assigned to a photopeak in the spectrum. PCGAP looks at the lines associated with each nuclide to determine if the intensity code 1 line has been associated with a photopeak. If it is not, all other associations with other lines from that nuclide will be deleted. PCGAP then prints a line in the output listing which indicates how many nuclide associations have been removed because an intensity code 1 line is not present. Figure 4 is an example of the nuclide summary section of the PCGAP output.

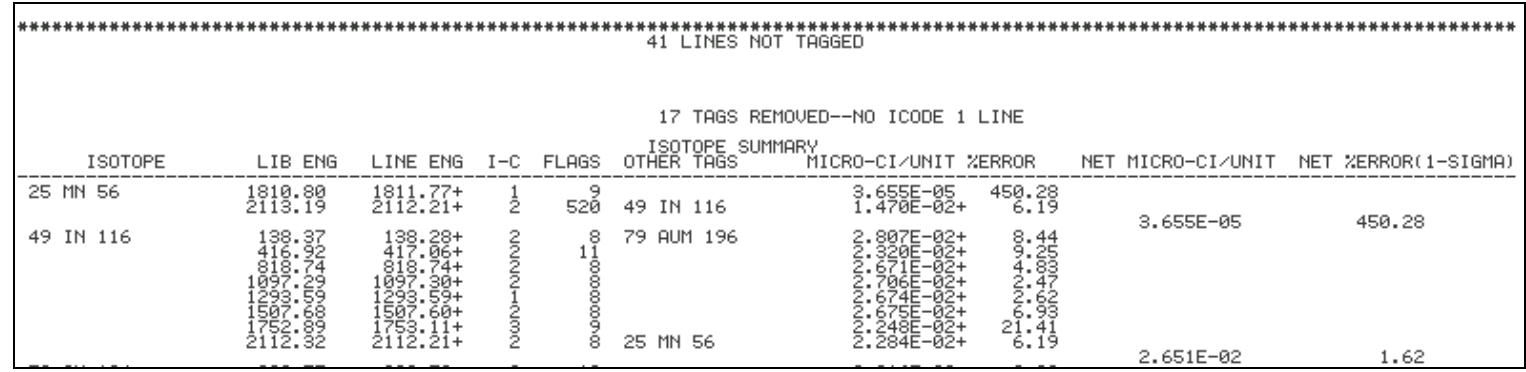

Figure 4. Nuclide Summary Section.

In this section all the lines for each identified radionuclide are grouped together and printed under the following heading:

ISOTOPE | LIB ENG | LINE ENG | I-C | FLAGS | OTHER TAGS | MICRO-CI/UNIT | \%ERROR | NET MICRO-CI/UNIT | NET \%ERROR(1-SIGMA)

The definition of these headings are as follows:

ISOTOPE... Is the atomic number, Chemical symbol and atomic weight of the radioisotope.

LIB ENG... This is the nuclide line energy as it exists in the nuclide library.

LINE ENG... This is the computed energy of the photopeak. It is computed from the centroid of the photopeak and the values of energy equation coefficients stored in the parameter record of the spectrum.

I-C... This integer value is the nuclide line intensity code. 
FLAGS...This integer describes how the photopeak was fit and indicates other operations which might have been performed on the peak area. See Appendix B for a description of possible flag values.

OTHER TAGS... This column contains the atomic number, Chemical symbol and atomic weight of other radionuclides which are in the nuclide library and have a line which is within $1 \mathrm{keV}$ of the Line energy value.

MICRO-CI / UNIT \%ERROR... This is the activity concentration value in units of micro curies and its associated error (expressed as a percent) from each photopeak. It is derived from the area of the photopeak with modifications as described by the FLAG value.

NET MICRO-CI / UNIT ... This value is net activity concentration of the radionuclide. This value is computed by performing a weighted average, with outlier testing, of all the lines associated with the nuclide.

\subsection{Fitting Algorithms}

PCGAP uses two methods to determine photopeak areas:

1. A nonlinear fitting of a Gaussian function to a photopeak in which the width, position (centroid), and height of the Gaussian function are allowed to vary, but the contiguous background under the peak is determined independently and is not part of the fit. The nonlinear fitting process is applied to those photopeaks that have been located by the photopeak search algorithm.

2. A linear fitting of the photopeak to a Gaussian function (with the position and width fixed) and a linear background. The linear fitting process is used for photopeaks that can not be successfully fit by the nonlinear method and for fitting regions, of the spectrum where a required photopeak would be located if it was present, but was not detected by the photopeak search algorithm. This capability (to fit designated regions of the spectrum) is provided to obtain analytical results for a set of required radionuclides (See required energy library). As an example, suppose that the concentration of ${ }^{131} \mathrm{I}$ in an air filter must be determined, regardless of its activity level; such a directed analysis capability is then required. The determination of activity concentration values for a set of required radionuclide will be denoted as "directed analysis" in subsequent discussions.

The use of these two photopeak fitting methods is exclusive. If the nonlinear fitting of a photopeak is successful, the photopeak is not refit by the linear process. 


\subsubsection{Nonlinear Fit Procedure}

Before beginning a discussion of the algorithms used to fit peaks it is appropriate to describe the notations used. The width function used in PCGAP is a linear equation of the form:

$$
\mathrm{W}(\mathrm{x})=\mathrm{A}+\mathrm{B} \bullet \mathrm{x}
$$

Where $\mathrm{x}$ is a channel number.

The values for the coefficients A and B are determined by the spectrum energy calibration function of PCGAP. W(x) returns the FWHM of a photopeak (in channels) at channel $\mathrm{x}$. The expression $\mathrm{W}(\mathrm{x})$ will be used to denote the value of the width equation for a peak whose centroid is at channel $\mathrm{b}$.

The nonlinear least-squares fitting procedure minimizes the sum of the squares of the deviation of data from a Gaussian function form. In analytical terms, the procedure determines the values of the height at the centroid $(\mathrm{H})$, full width at half maximum $(\mathrm{W})$, and the Centroid $(\mathrm{C})$ of a Gaussian function that minimizes:

$$
R^{2}=U_{i}\left(Y_{i}-G_{i}\right)^{2}
$$

Where the indicated summation is from the lower to upper fit limits (established as detailed in the following section). $Y_{i}$ represents the spectral data at channel $i$ and $U_{i}$ is a statistical weighting term. The Gaussian function $\mathrm{G}_{\mathrm{i}}$ is defined below.

$$
G_{i}=H \bullet e^{\left(-4 \ln 2\left((i-C)^{2}\right) / W^{2}\right.}
$$

The nonlinear fitting procedure can fit up to five photopeaks simultaneously using a common spectral background. The criteria used to determine whether or not a multiplet fit is to be performed depends on how many full widths exist between the photopeak being fit and the next photopeak. Let $\mathrm{X}_{\mathrm{i}}$ be the ith peak location to be fit and $X_{i+1}$ be the next photopeak location. Then if

$$
\mathrm{X}_{\mathrm{i}+1}-\mathrm{X}_{\mathrm{i}}<2.5 \bullet \mathrm{W}\left(\mathrm{X}_{\mathrm{i}}\right) \text { Where } \mathrm{X}_{\mathrm{i}}<\mathrm{X}_{\mathrm{i}+1}
$$

the photopeaks $\mathrm{X}_{\mathrm{i}}$ and $\mathrm{X}_{\mathrm{i}+1}$ are fit together. This process continues until a maximum of five photopeaks are grouped together for a multiplet fit.

Two regions must be determined before a photopeak can be fit. The first region defines the channels over which the least-squares fit is to be performed. The second is the region over which a background will be determined (see Figure 5).

The first channel of the fit (FCF) and the ending channel of the fit (ECF) for a peak located at $\mathrm{X}_{\mathrm{i}}$ are defined as follows:

$$
\begin{aligned}
& \mathrm{FCF}=\mathrm{X}_{\mathrm{i}}-7.5 \bullet \mathrm{W}\left(\mathrm{X}_{\mathrm{i}}\right) \\
& \mathrm{ECF}=\mathrm{X}_{\mathrm{i}}+7.5 \bullet \mathrm{W}\left(\mathrm{X}_{\mathrm{i}}\right)+1.7
\end{aligned}
$$




\subsubsection{Determination of Spectrum Contiguous Background For A Nonlinear Fit.}

The spectrum background under a photopeak to be fit by the nonlinear process is assumed to be represented by a linear function:

$$
\mathrm{BK}(\mathrm{x})=\mathrm{A}+\mathrm{B} \bullet \mathrm{x}
$$

Where $\mathrm{x}$ is a channel number

The linear function's coefficients are determined by examining the spectrum contents outside the fit limits. Once the coefficients of the background lines have been determined, channel counts in the fitting region will be reduced by the background line value evaluated at each channel in the fit region. In analytical terms:

Let $\mathrm{C}_{\mathrm{i}}$ be the counts in channel $\mathrm{i}$ and $\mathrm{BK}(\mathrm{i})$ be the background equation defined above. Then the net count, $Y_{i}$, is defined by

$$
\mathrm{Y}_{\mathrm{i}}=\mathrm{C}_{\mathrm{i}}-\mathrm{BK}(\mathrm{i})
$$

For all channels in the region FCF to ECF.

The coefficients $(\mathrm{A}, \mathrm{B})$ for the background equation are the coefficients of a line drawn between average background values determined on the high and low sides of the photopeak being fit.

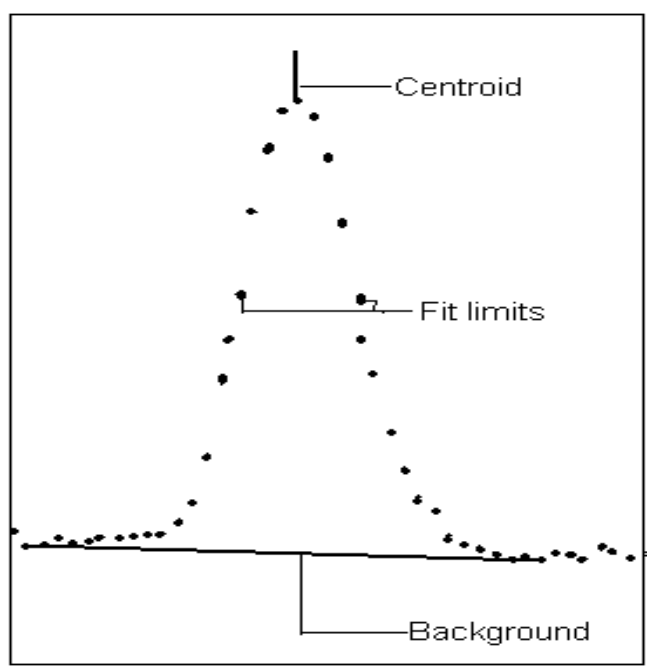

Figure 5. Background and fit limits. The y axis is $\log \left(\mathrm{C}_{\mathrm{i}}\right)$.

The average background value on the low-energy side of the photopeak is established by examining the spectral contents between the lower background limit (LBL) and the first channel of the fit. The lowest channel value in this region is located and the average of this value and the two adjacent channel values is computed to establish an initial background value. This initial background value is augmented by including in the background those contiguous channels that have calculated one-sided $99 \%$ confidence intervals that include the initial background value. The high-side background value is determined in the same manner, but the region from the last channel of the fit to the upper background limit (UBL) is the region which is examined. The value for LBL is the channel number that is five widths from the centroid of the photopeak: 
where

$$
\mathrm{LBL}=\mathrm{X}_{\mathrm{i}}-5 \bullet \mathrm{W}\left(\mathrm{X}_{\mathrm{i}}\right)
$$

$$
\mathrm{X}_{\mathrm{i}}=\text { photopeak centroid }
$$

The spectrum's contents in the region LBL to FCF are examined to determine the channel, $\mathrm{X}$, having the smallest number of counts. An average, Av, of the counts in channels $\mathrm{X}-1, \mathrm{X}$, and $\mathrm{X}+1$ is then computed. This is the initial background value. To determine if the contents, $\mathrm{Y}_{\mathrm{i}}$ at channel $\mathrm{i}$, (contiguous to the $\mathrm{X} \pm 1$ channel region used to define the initial background value) should be included in the background average, a test is performed to determine if $\mathrm{Av} \# \mathrm{SS}_{\mathrm{i}}$, where the test statistic, $\mathrm{SS}_{\mathrm{i}}$, is the estimated lower limit of a one-sided $99 \%$ confidence interval constructed about $\mathrm{Y}_{\mathrm{i}}$. This procedure allows all channel contents between the LBL and the start of the fit limit to be included in the background average if the background region is flat and all values truly represent the background, but it avoids establishing an erroneously high background in the presence of interference features.

The method used to establish $\mathrm{SS}_{\mathrm{i}}$ depends on the number of counts, $\mathrm{Y}_{\mathrm{i}}$, in channel i. If $\mathrm{Y}_{\mathrm{i}}$ is greater than 20, then the normal assumption is applied and $\mathrm{SS}_{\mathrm{i}}=2.32 *\left(\mathrm{Y}_{\mathrm{i}}\right)^{1 / 2}$. If $\mathrm{Y}_{\mathrm{i}}$ is less than or equal to 20, Poisson statistics are assumed to apply and the following table is used to estimate $\mathrm{SS}_{\mathrm{i}}$.

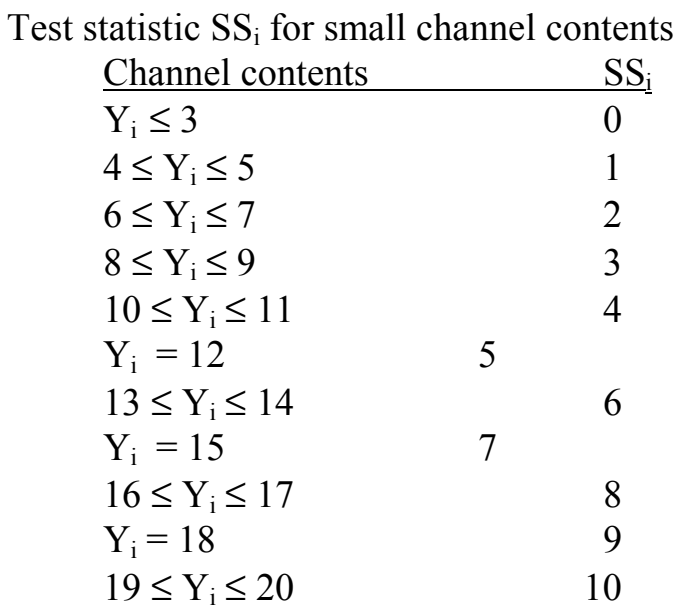

\subsubsection{Photopeak Area and Error Determination.}

Once a nonlinear fit has been made of the Gaussian function(s) to the data, the photopeak area can be computed.

where

$$
\text { Area }=W \bullet H \bullet \sqrt{\pi / 4 \ln 2}
$$

$$
\begin{aligned}
& \mathrm{W}=\text { the Gaussian width at half maximum, } \\
& \mathrm{H}=\text { the Gaussian height. }
\end{aligned}
$$

The estimated variance in the final values of the Gaussian parameters (by analogy with linear analysis) are calculated from the elements of the final inverse matrix. Because the photopeak area is derived from two parameters (Gaussian width and height), its estimated variance, (Area), includes a covariance term, COVAR:

$$
\sigma^{2}(\text { Area })=\frac{R^{2}}{m \bullet I N V \bullet C O V A R}
$$

where 
$\sigma^{2}$ (Area) $=$ The estimated variance of the area

$\mathrm{m}=$ The number of degrees of freedom

$\mathrm{R}^{2}=$ The sum of the squares of the residuals

$\mathrm{INV}=$ The sum of the height and width squared times their respective inverse matrix elements.

The percent of error in the area is then defined to be:

$$
\% \operatorname{Error}(\text { Area })=\frac{\sqrt{\sigma^{2}(\text { Area })}}{\text { Area }} \bullet 100
$$

\subsubsection{The Linear Fit Procedure}

The linear fitting procedure is used to compute photopeak areas for photopeaks for which the nonlinear fitting process failed, and to fit selected regions of the spectrum to determine activity concentration values for a set of preselected, required radionuclides.

The linear least squares procedure assumes that a photopeak is represented by a Gaussian function and a linear background. The position and width of the Gaussian are fixed during the fitting process.

Centroids of photopeaks to be fit are determined either from the initial centroid as determined by the photopeak search algorithms for a failed nonlinear fit or from a list of required energy values. The energy values are read from the required energy file and converted to a channel location by using the energy equation. Spectral data surrounding the derived channel location are used in the linear fitting process.

Once the linear fitting region has been determined, it is assumed that a Gaussian function, Gi, and a linear background represent the peak. The data is fit to determine coefficients $a, b$, and $\mathrm{H}$.

where

$$
\mathrm{Y}_{\mathrm{i}}=\mathrm{a}+\mathrm{bX} \mathrm{X}_{\mathrm{i}}+\mathrm{HG}_{\mathrm{i}}
$$

$$
\begin{aligned}
& \mathrm{a} \text { and } \mathrm{b}=\quad \text { the coefficients of the linear background line, and } \\
& \mathrm{H}=\text { the height of the Gaussian function. }
\end{aligned}
$$

The area of the photopeak is then computed using the area equation determined in Section 3.2.1.2.

The error in the area is computed using the following:

$$
\sigma(\text { Area })=\sqrt{\pi / 4 \ln 2} \bullet \chi \bullet m
$$

where

$\chi=$

the square root of the sum of the squares of the residuals divided by the number of channels in the fit, and

$\mathrm{m}=$

derived from the appropriate minor of the inverse matrix for the Gaussian height variable in the fit. 


\subsubsection{Linear Fit Region Determination.}

The fit region used in a linear fit is determined from the position of the photopeak to be fit and the surrounding spectrum structure. This region must contain enough of the spectrum to determine the continuum background. However, to avoid an erroneous background definition, the selected region must not overlap a region of the spectrum that contains another photopeak. To determine this fitting region, several tests are performed on the location of photopeaks that surround the photopeak to be fit.

Let

$X_{i}=$ The Centroid of photopeak to be fit

$X_{i-1}=$ The centroid of the peak that immediately precedes $X_{i}$

$X_{i+1}=$ The centroid of the photopeak that immediately follows $X_{i}$

$\mathrm{LF}=$ Beginning channel of the fit region surrounding $X_{\mathrm{i}}$

$\mathrm{UF}=$ Ending channel of the fit region surrounding $\mathrm{X}_{\mathrm{i}}$

$\mathrm{E}_{\mathrm{i}}=$ The energy of the photopeak at $\mathrm{X}_{\mathrm{i}}$

$\mathrm{W}\left(\mathrm{X}_{\mathrm{i}}\right)=$ Value of width equation at $\mathrm{X}_{\mathrm{i}}$

Then

Test Resulting Fit Limits

$\mathrm{X}_{\mathrm{i}-1}+5 \bullet \mathrm{W}\left(\mathrm{X}_{\mathrm{i}-1}\right)<\mathrm{X}_{\mathrm{i}}<\mathrm{X}_{\mathrm{i}+1}-5 \bullet \mathrm{W}\left(\mathrm{X}_{\mathrm{i}+1}\right)$

$\mathrm{LF}=\mathrm{X}_{\mathrm{i}}-2 \bullet \mathrm{W}\left(\mathrm{X}_{\mathrm{i}}\right)$

$\mathrm{UF}=\mathrm{X}_{\mathrm{i}}+2 \bullet \mathrm{W}\left(\mathrm{X}_{\mathrm{i}}\right)$

$\mathrm{X}_{\mathrm{i}-1}+5 \bullet \mathrm{W}\left(\mathrm{X}_{\mathrm{i}-1}\right)<\mathrm{X}_{\mathrm{i}}<\mathrm{X}_{\mathrm{i}+1}-3 \bullet \mathrm{W}\left(\mathrm{X}_{\mathrm{i}+1}\right)$

$\mathrm{LF}=\mathrm{X}_{\mathrm{i}}-3 \bullet \mathrm{W}\left(\mathrm{X}_{\mathrm{i}}\right)$

$\mathrm{UF}=\mathrm{X}_{\mathrm{i}}+\mathrm{W}\left(\mathrm{X}_{\mathrm{i}}\right)$

$\mathrm{X}_{\mathrm{i}-1}+2.5 \bullet \mathrm{W}\left(\mathrm{X}_{\mathrm{i}-1}\right)<\mathrm{X}_{\mathrm{i}}<\mathrm{X}_{\mathrm{i}+1}-5 \bullet \mathrm{W}\left(\mathrm{X}_{\mathrm{i}+1}\right)$

$\mathrm{LF}=\mathrm{X}_{\mathrm{i}}-\mathrm{W}\left(\mathrm{X}_{\mathrm{i}}\right)$

$\mathrm{UF}=\mathrm{X}_{\mathrm{i}}+3 \bullet \mathrm{W}\left(\mathrm{X}_{\mathrm{i}}\right)$

$\left|E_{i}-E_{i+1}\right|<1$

Fit is not performed

$\left|\mathrm{E}_{\mathrm{i}}-\mathrm{E}_{\mathrm{i}-1}\right|<1$

Fit is not performed

All other cases

$$
\begin{aligned}
& \mathrm{LF}=\mathrm{X}_{\mathrm{i}}-2 \bullet \mathrm{W}\left(\mathrm{X}_{\mathrm{i}}\right) \\
& \mathrm{UF}=\mathrm{X}_{\mathrm{i}}+2 \bullet \mathrm{W}\left(\mathrm{X}_{\mathrm{i}}\right)
\end{aligned}
$$

The tests are performed to determine the linear fit limits, LF and UF, and are performed in the order listed. The fit limits are established as defined for the first test that is true. Once a test is satisfied, the testing procedure ends and the fitting process begins using the resulting fit limits.

The testing process begins by checking the position of the photopeak in relation to the photopeaks surrounding it. If there is no photopeak within five widths of the photopeak position, it is assumed that there will be no interferences and the photopeak will be fit within a symmetrical region of two widths from the photopeak position, i.e., two widths on either side of the photopeak centroid.

For the second and third tests, it is assumed that photopeak interference exists. The interference can be reduced to acceptable levels by shifting the limits of the fitting region away from the interference photopeak. The fitting region will be skewed to the side opposite the interference photopeak. 
The fourth and fifth tests determine if the photopeak to be fit is within 1-keV of an interference photopeak. If it is, no fit is made.

\subsection{Nuclide Identification}

Radionuclide identification of photopeaks present in a spectrum is done by matching the energy of the photopeak to a list of energies in a nuclide library. A spectral photopeak is assigned a potential nuclide identification if the library entry for that nuclide is within one $\mathrm{keV}$ of the spectral photopeak energy. A maximum of twenty nuclide tags can be assigned to any one photopeak. If there is no entry in the nuclide identification library within one $\mathrm{keV}$ of the spectral photopeak energy, no nuclide identification is made and no further computations are performed on that photopeak.

A nuclide library is a list of gamma-ray energies with nuclide identifications. Each entry identifies the nuclide, its associated gamma-ray energy, error in the energy, emission probability (branching ratio), error in branching ratio, half-life, and an intensity code (1 to 5) indicating the relative worth of that specific photopeak in the decay of the nuclide. The most prominent and indicative line for each isotope has an intensity code (IC) of 1.

Since applications can require the use of nuclide libraries containing different entries, different libraries can be constructed and stored as disk files. Most spectrum analysis procedures in the PCGAP package require a nuclide library file name as part of the input menu. Utility programs can be used to construct and modify nuclide libraries.

The fitting process initiates a data structure for each photopeak as it is fit. The structure initially includes the area, energy, width, and channel location of the photopeak. If a radionuclide is identified for a photopeak, then an entry is made in a nuclide data structure. This nuclide data structure includes:

1. Nuclide name, atomic number, and mass.

2. Nuclide branching radio and half-life.

3. Calculated decay correction for that nuclide.

4. Nuclide photopeak intensity code.

5. Count rate (counts/second) of the isotope. This rate is computed from the photopeak area and the live-time, and is corrected for pulse pile-up losses. In PCGAP, the pulse pile-up correction (for systems that don't incorporate pulse injection) is performed using a linear relationship of the total counts in the spectrum, and is measured independently for each spectrometer system. In systems using pulser injection with subsequent removal, spectrumspecific pulse pileup corrections are determined from the pulser data.

\subsection{Decay Correction Calculation}

The calculated decay correction is the product of two parameters. The first parameter, C1, provides the correction for the decay incurred from the time the sample was collected to the start of the count and for the decay during the counting interval. The second parameter, $\mathrm{C} 2$, is primarily for air sampling filters and provides correction for decay (if any) incurred during the sample collection.

The decay contribution, $\mathrm{C} 1$, for the interval from sampling though the counting interval, is computed as follows: 
Let

$\mathrm{T}_{\mathrm{R}}=$ real time during the count

$\lambda=.69314 /$ half-life of nuclide

$\mathrm{T}_{\mathrm{d}}=$ decay time from removal of sample from sample stream to the start of the count.

Then

$$
C 1=\lambda T_{R}\left[e^{-\lambda T_{d}}-e^{-\lambda\left(T_{d}+T_{1}\right)}\right]^{-1}
$$

The second term of the decay correction, C2, that corrects for the decay during the sampling interval, is calculated as follows:

Let

$$
\mathrm{T}_{\mathrm{s}} \quad=\quad \text { sampling duration. }
$$

Then

$$
C 2=\frac{\lambda \bullet T_{s}}{\left(1-e^{-\lambda \bullet T_{s}}\right)}
$$

The product of $\mathrm{C} 1$ and $\mathrm{C} 2$ is the decay correction factor that is applied to a radionuclide.

PCGAP will remove a nuclide identification for a photopeak when the decay correction factor to be applied is excessive. A nuclide identification will be removed if either of the following tests are true:

$$
\begin{array}{ll}
\text { 1. } & T_{d} * \lambda>15 \\
\text { 2. } & \mathrm{C} 1 \bullet \mathrm{C} 2>1000 .
\end{array}
$$

\subsection{Interference Decontamination Algorithm}

In a gamma-ray pulse-height spectrum, it is possible that two or more gamma-rays from different radionuclides have contributed to a single photopeak. In PCGAP, an interference decontamination algorithm determines which fraction of a single contaminated photopeak area belongs to each of the contributing nuclides, provided some or all radionuclides involved have "clean" photopeaks to provide interference information. Given the outlier testing and averaging implemented in the PCGAP code it is generally not required that all interferences be identified and corrected. It is usually sufficient to decontaminate the $\mathrm{IC}=1$ line for each nuclide of interest from possible interferences. For this algorithm, the user of the system must know what interferences could be present in the sample and, through the use of an interference decontamination library, indicate how the interferences can be resolved. The interference library is a list of energies and nuclide gamma-ray photopeaks that may be contaminated, plus the energies of clean lines that can be used to decontaminate the contaminated lines. As an example, a typical library might contain information as shown in Table 1. 
Table 1. Interference Decontamination

\begin{tabular}{|c|c|c|c|c|c|c|c|c|c|}
\hline \multirow[b]{2}{*}{ Energy } & \multicolumn{4}{|c|}{ Contaminated Lines } & \multicolumn{5}{|c|}{ Clean Lines } \\
\hline & & Z Element & $\begin{array}{l}\text { Atomic } \\
\text { Number }\end{array}$ & Isomer & Energy & & Element & $\begin{array}{l}\text { Atomic } \\
\text { Number }\end{array}$ & Isomer \\
\hline 834.827 & 25 & $\mathrm{Mn}$ & 54 & NO & 2392.0 & 36 & $\mathrm{Kr}$ & 88 & NO \\
\hline 772.600 & 53 & I & 132 & NO & 479.51 & 74 & $\mathrm{~W}$ & 187 & NO \\
\hline 810.757 & 27 & $\mathrm{Co}$ & 58 & NO & 772.60 & 53 & $\mathrm{I}$ & 132 & NO \\
\hline
\end{tabular}

Consider the first line in the library of Table $1 .{ }^{54} \mathrm{Mn}$ at $834.827 \mathrm{keV}$ is contaminated by ${ }^{88} \mathrm{Kr}$. To decontaminate it, the ${ }^{88} \mathrm{Kr}$ clean line (at $2392.020 \mathrm{keV}$ ) will be used to determine the area of 834.827 $\mathrm{keV}$ peak contributed by ${ }^{88} \mathrm{Kr}$. The actual calculation is described below in detail.

The decontamination algorithm allows both multiple and indirect decontaminations. In a multiple decontamination, a single photopeak is decontaminated from more than one isotope. In an indirect decontamination, a contaminated photopeak is decontaminated and the result used to decontaminate another photopeak. The decontaminations will be done in the order listed in the interference library.

The number of decontaminations done in a multiple decontamination is limited by the maximum number of isotope tags that a photopeak can receive by the nuclide identification routine. Therefore, the number of decontaminations that can be done is limited to 19 .

In practice, almost any number of indirect decontaminations can be done, i.e., nuclide A can be used to decontaminate nuclide $\mathrm{B}$, which can be used to decontaminate nuclide $\mathrm{C}$, which can be used to decontaminate nuclide $\mathrm{D}$. The only limit is the size of the interference library. The current library has room for a maximum of 50 entries. Each single decontamination, or each step in a multiple or indirect decontamination, requires one entry in the library.

Calculating disintegrations per second as photopeak count rate (cps) divided by the photopeak efficiency and the branching ratio, the net corrected disintegrations per second, DPS $\mathrm{Net}_{\mathrm{N}}$ at the contaminated line energy is:

$$
D P S_{\text {Net }}=D P S_{\text {contaminated }}-D P S_{\text {clean }} \frac{B R_{\text {Clean }}}{B R_{\text {contaminated }}}
$$

where

$\mathrm{DPS}_{\text {contaminated }}=\quad \begin{aligned} & \text { the disintegration rate of the contaminated isotope calculated } \\ & \text { from the contaminated line, } \\ & \text { the disintegration rate of the contaminating isotope calculated } \\ & \text { from the peak at the clean energy, }\end{aligned}$
$\mathrm{BR}_{\text {Clean }}=\quad \begin{gathered}\text { the branching ratio (fractional) of the contaminating nuclide to } \\ \text { the contaminated energy, and }\end{gathered}$
$\mathrm{BR}_{\text {Contaminated }}=\quad$ the branching ratio (fractional) of the contaminated nuclide to
the contaminated energy

The estimated standard deviation, $\sigma_{\mathrm{Net}}$, in the $\mathrm{DPS}_{\mathrm{Net}}$ is: 


$$
\sigma_{\text {Net }}=\sqrt{\sigma_{\text {contaminated }}^{2}+\left(\sigma_{\text {Clean }}^{2}+\frac{B R_{\text {Clean }}^{2}}{B R_{\text {contaminated }}^{2}}\right)^{2}}
$$

where

$$
\begin{aligned}
& \sigma_{\text {Contaminated }}=\quad \text { estimated standard deviation of the line at the contaminated energy } \\
& \sigma_{\text {Clean }}=\quad \text { estimated standard deviation of the line at the clean energy. }
\end{aligned}
$$

\subsection{Weighted Average and Outlier Testing Procedure}

For nuclides having more than one associated photopeak with one assignment having an intensity code of 1 , a single weighted average concentration value is computed. The weighted average is derived from the set of concentration values computed from each of the individual photopeaks. An outlier test is performed on the set of concentration values and photopeaks that do not pass the outlier test are not used in the weighted average. The weighted average is performed using photopeak concentrations of microcuries per unit volume. All individual concentration values are printed, along with the computed weighted average.

The following procedure is followed to determine the net microcurie value for a nuclide from a set of individual results [see Equation (26)]. For $\mathrm{N}$ results, calculate the weighted average, $\bar{X}$, and the estimated standard deviation, $\bar{S}$ :

$$
\begin{aligned}
& \bar{X}={ }_{i=1}^{N} \frac{w_{i} \bullet X_{i}}{N} w_{i} \\
& \bar{S}=\frac{\sqrt{i=1}{ }_{i=1}^{N} w_{i}^{2} \bullet \sigma_{i}^{2}}{w_{i}}
\end{aligned}
$$

Where

$$
\begin{aligned}
\mathrm{X}_{\mathrm{i}} & =\text { Microcurie value of photopeak } \mathrm{i} . \\
\sigma_{\mathrm{i}} & =\text { The estimated standard deviation in } \mathrm{X}_{\mathrm{i}} \text {. (See section 3.2.1.2) } \\
\mathrm{W}_{\mathrm{i}} & =\sqrt{\frac{1}{\sigma_{i}^{2} \bullet I C_{i}}} \text { Where the } \mathrm{IC}_{\mathrm{i}} \text { is the intensity code of the photopeak }
\end{aligned}
$$

The reduced chi-squared $\chi_{R}{ }^{2}$, is now computed and used as an outlier criterion. 


$$
\chi_{R}^{2}=\frac{1}{D F}_{i=1}{ }^{N}\left(\frac{X_{i}-\bar{X}}{\sigma_{i}}\right)^{2}
$$

Where DF $=\mathrm{N}-1$ if $\mathrm{N}>3$

$$
=\mathrm{N} \text { if } \mathrm{N} \leq 3
$$

If $\chi_{R}^{2} \leq 4$ then the set of values is deemed consistent and the net concentration,

$$
\mathrm{C}_{\mathrm{net}}=\bar{X}
$$

with a percent error, $\% \mathrm{E}$, of

$$
\begin{array}{ll}
\% \mathrm{E}=100.0 \cdot \frac{\bar{S}}{\bar{X}} \quad \text { if } \quad \chi_{\mathrm{R}}^{2} \leq 1.0 \\
\% \mathrm{E}=100.0 \cdot \frac{\bar{S}}{\bar{X}} \cdot \sqrt{\chi_{R}^{2}} \quad \text { if } \chi_{\mathrm{R}}^{2} \geq 1.0
\end{array}
$$

If $\chi_{R}^{2}>5$ and $N=2$ then

$$
\begin{gathered}
\mathrm{C}_{\mathrm{net}}=\text { smallest microcurie value } \mathrm{X}_{\mathrm{i}} \text { and a percentage error, } \\
\qquad \% \mathrm{E}=\frac{\sigma_{i}}{X_{i}} \bullet 100.0
\end{gathered}
$$

If $\chi_{\mathrm{R}}^{2}>5$ and $\mathrm{N}>2$ then the photopeak with the largest value for $\mathrm{X}_{\mathrm{a}}$,

$$
X_{a}=\frac{\left|X_{i}-\bar{X}\right|}{\sigma_{i}} \text { is deemed an outlier and is discarded. }
$$

The value for $\mathrm{N}$ is reduced by one and the weighted average and outlier test procedure is repeated. 


\subsection{ENERGY AND WIDTH EQUATIONS}

The energy scale in PCGAP is expressed as a quadratic equation of the form

$$
\mathrm{E}(\mathrm{x})=\mathrm{A}+\mathrm{B}(\mathrm{x})+\mathrm{C}\left(\mathrm{x}^{2}\right)
$$

Where $\mathrm{x}$ is a channel number.

A photopeak width equation, $\mathrm{W}(\mathrm{x})$, used in PCGAP to determine the initial width value for the peak fitting processes is a linear function of the form:

$$
\mathrm{W}(\mathrm{x})=\mathrm{F}+\mathrm{G}(\mathrm{x})
$$

Where $\mathrm{x}$ is a channel number.

The energy equation parameters $(\mathrm{A}, \mathrm{B}$, and $\mathrm{C})$ and the width parameters $(\mathrm{F}$ and $\mathrm{G})$ can be determined using options in the PANDA program (Appendix E, section E-4). 


\subsection{DETECTOR EFFICIENCY TABLES}

In PCGAP the method used to derive nuclide quantitative values requires the use of an efficiency table. A different table must be created for every different geometric and detector configuration An efficiency table is a set of ordered pairs (energy, efficiency). The nuclide concentration at a particular energy, $\mathrm{C}(\mathrm{E})$, is then determined using the following:

$$
C(E)=\frac{c p s \bullet d c}{\text { eff } \bullet \text { brf } \bullet 3.7 E+4 \bullet \text { volume }}
$$

Where:

cps $=$ The photopeak counts/second value corrected for ambient background contribution and random summing.

$\mathrm{dc}=$ decay correction (see section 3.4.1 Decay Correction Calculation) for the particular nuclide. volume $=$ Volume of the sample

brf $=$ The branching ratio of the nuclide line (expressed as a fraction).

eff $=$ The efficiency value at the photopeak energy (counts/gamma).

The efficiency value extracted from the efficiency table is determined by locating the entry in the table for the energy of the energy-efficiency pair which is closest to the photopeak energy, call it $\left(\mathrm{e}_{\mathrm{i}}, \mathrm{eff}_{\mathrm{i}}\right)$. Then performing a parabolic interpolation between the pairs of points, $\left(\mathrm{e}_{\mathrm{i}-1}, \mathrm{eff}_{\mathrm{i}-1}\right),\left(\mathrm{e}_{\mathrm{i}}, \mathrm{eff}_{\mathrm{i}}\right),\left(\mathrm{e}_{\mathrm{i}+1}, \mathrm{eff}_{\mathrm{i}+1}\right)$.

Efficiency table files have a specific naming convention, which must be adhered to in order for the file to be opened correctly by the spectrum analysis codes. The name is constructed from a string of ten characters: ttddddnnnn where $\mathrm{tt}$ is the detector identification dddd is the source to detector distance and nnnn is a four digit table number. The extension used for these files is ".eff". Some examples of valid efficiency file names are:

A200001413 Efficiency for detector A2, source to detector distance is zero and the table number is 1413 .

S101000014 Efficiency for detector S1, source to detector distance is 100 and the table number is 14 . 


\subsection{MISCELLANEOUS UTILITY PROGRAMS}

The following functions are an integral part of PCGAP:

- Generation of efficiency tables using (1) a text input file and (2) a transform method.

- Creation of nuclide libraries.

- Spectrum file format conversion from ".chn, .rpt" and other file types to PCGAP format.

- Two functions for the; (1) Editing, printing and creation of required energy libraries and (2) Printing of output listing files.

The following sections describe the utility programs used to perform these functions. A short cut to each of the utility executables is created when PCGAP is installed. In case the short cuts have disappeared, this description will refer to the utility programs by the name of the executable for that program. The executables can be found in pcgaplexe directory.

\subsection{Generation of Efficiency Tables}

Efficiency tables can be created using two different programs: (1) Txt_eff.exe which uses a text file as input to generate the efficiency table and (2) Efficiency.exe, which creates efficiency tables from a spectrum accumulated with a set of standard sources with known activities.

\subsubsection{Txt_eff .}

The use of this utility requires the user to create a text file containing ordered pairs of energy and efficiency at that energy. Notepad or equivalent text editor can be used to create these files. The following is an example of a set of energy, efficiency pairs:

\begin{tabular}{|c|c|}
\hline Energy & Efficiency \\
\hline 50.0 & $3.8898 \mathrm{E}-3$ \\
\hline 55.0 & $7.3522 \mathrm{E}-3$ \\
\hline 60.0 & $1.0569 \mathrm{E}-2$ \\
\hline 65.0 & $1.3635 \mathrm{E}-2$ \\
\hline
\end{tabular}

Here we have efficiency values for $50,55,60,65 \mathrm{keV}$. There is no required energy value separation; values can be as close or as far apart as the user desires. The text file can contain up to 300 ordered pairs.

The user will be asked to supply the name of the input text file and the efficiency table name. The name must adhere to the PCGAP naming convention (See section 5.0).

\subsubsection{Efficiency}

The efficiency program begins execution by displaying a window with two active menus. 


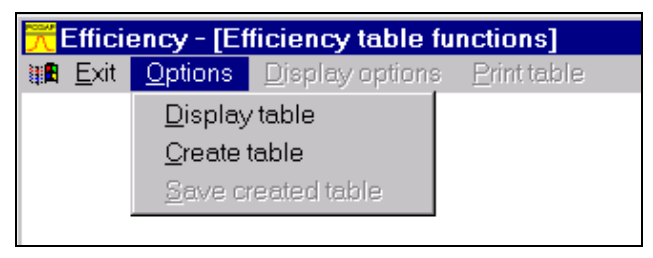

Figure 6. Efficiency start menus

The "Display table" option allows the user to plot the contents of any existing efficiency table. Selection of Display table will generate an open file dialog box from which the user selects the efficiency table to display. After the table has been selected the other menu items "Display options" and "Print table" will be enabled. Use of these menu functions is self-explanatory.

The Create table function implements a transform methodology to generate efficiency curves. The method executes the following steps:

1. Transforms the measured efficiency data into set of transform data, which has a monotonic decreasing dependence with gamma energy.

2. The transformed data are fit to a fourth order polynomial of the $\log _{10}$ of the energy versus the $\log _{10}$ of the efficiency.

3. The transformed fit equation is then converted back to an efficiency function that can be used to calculate the efficiency at any energy.

The beauty of this technique is its ease of use. A new efficiency curve can be generated in seconds once the standard source spectrum has been accumulated. The following steps must be performed to generate a new curve:

1. Collect a calibration spectrum using a set of calibration sources. In an ideal situation the photopeaks energies from the calibration sources should uniformly cover the energy range of interest. However, this is not an absolute requirement.

2. Prepare a standard file, which describes the calibration sources used. Selecting various options in the Create table function can be used to create the information in this file.

3. Use the Save created table function to save the generated the efficiency table on the disk.

The operator is not required to fit any curves or other wise manipulate any data. He is asked to supply the name of spectrum and standard file, a 4-digit number to assign to the efficiency file and the source to detector distance.

When the Create table function starts the following dialog box will be displayed: 


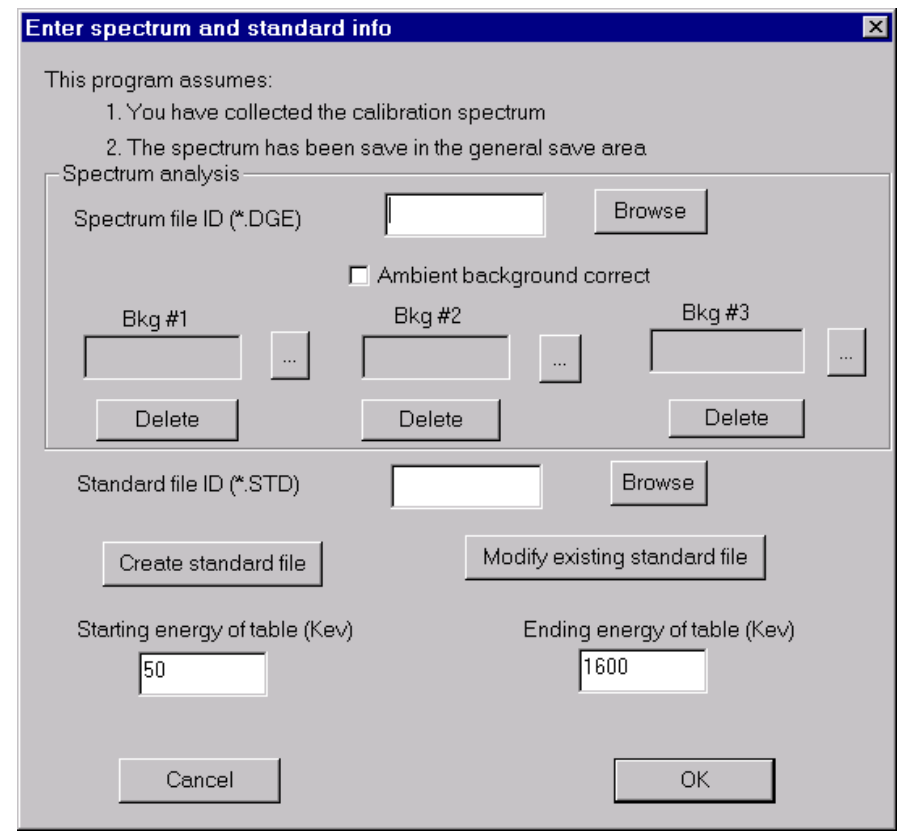

Figure 7. Create table Start Dialog.

Create table requires the input of two file names. The spectrum file ID which is the spectrum ID of the data collected with the standard sources. The identified spectrum is going to be analyzed to determine the efficiency values of the radionuclides present in the standard source; consequently the user can correct the spectrum results for the ambient background. If the ambient background correction option is selected up to three background spectra files can be used. These background files must be located in the BK save directory.

The Standard file ID is a file that contains information about the standard sources. Options exist with this dialog to create a new or modify an existing Standard file. Once a Standard file has been created for a set of sources it can be used whenever that set of sources is used to measure efficiencies. In other words, the Standard file doesn't have to be modified or created new every time the program is run, just when the characteristics of the standard sources change. Creation of a Standard file is described in Section 6.1.3 "Creating new Standard file". The user can also change the starting and ending energy range of the generated efficiency curve.

The user can use the browse buttons to select the spectrum and the standard files. Once the file ID's have been entered the generation of the curve can be initiated by clicking on "OK". The program will then analyze the spectrum and determine the efficiency values at the photopeak energies described in the Standard file. A printed output will be generated which will contain data on how the peaks in the spectrum were fit, backgrounds used, and the calculated efficiency values. The generated efficiency table files will be created in the PCGAP $\backslash$ DATA $\backslash$ EFFICIENCY directory.

After the analysis of the standard spectrum is completed an efficiency curve function is computed. A plot of the resulting efficiency values is then drawn in the active window. For example: 


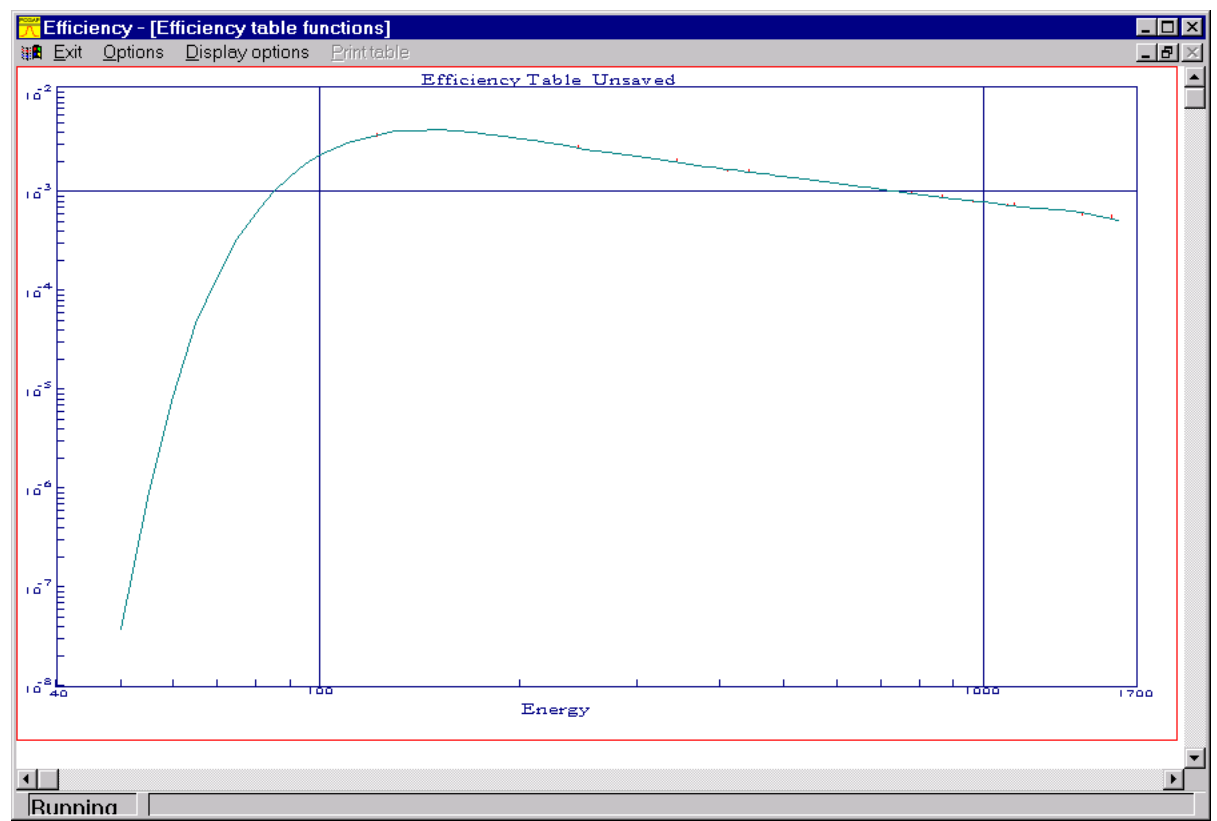

Figure 8. Generated efficiency curve.

The plot is generated from evaluating the efficiency curve at the following intervals:

\begin{tabular}{|l|l|}
\hline Evaluate every: & For the region \\
\hline $5 \mathrm{keV}$ & $<100 \mathrm{Kev}$ \\
\hline 10 & $<200$ \\
\hline 20 & $<500$ \\
\hline 50 & $>1000$ \\
\hline
\end{tabular}

The efficiency values (and associated error bars) which were computed for the photopeaks described in the Standard file are also plotted. The operator can then evaluate how good the fit of generated efficiency curve is to the standard photopeak values. The plot is a semi-log plot $\left(\log _{10}\right)$ of the Efficiency versus Energy.

After the newly created efficiency table data has been displayed the Save created table function under options menu will be enabled. If you do not select the save table function the table will not be permanently saved. Selection of Save created table function will generate the following dialog:

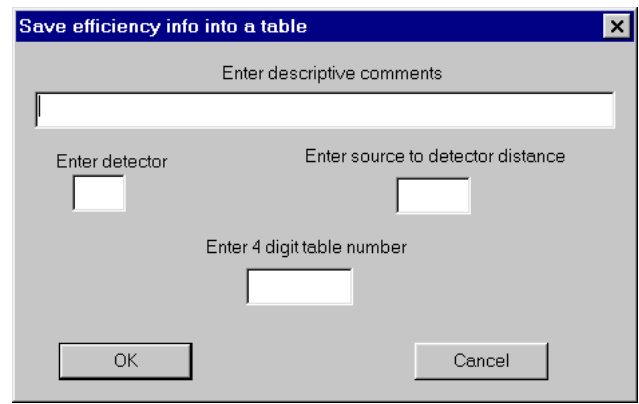

Figure 9. Save efficiency table dialog. 
The user can enter descriptive comments about the curve. These comments will be printed on any spectrum analysis when the new efficiency table is used.

The operator must enter the detector name, distance and efficiency table numbers and a table will be created from the entered values. When entering values for distance and table number DO NOT enter leading zeros. The program will supply them for you. When you select "OK" the "verify" dialog box will appear. For example:

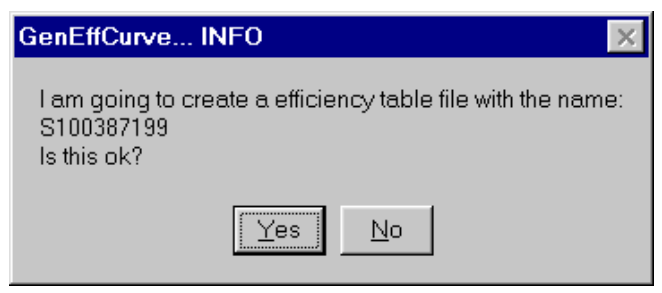

Figure 10. Efficiency file name verification.

If the "No" button is clicked the save dialog to reappear and new values can be entered. If "Yes" is selected the efficiency table file will be created.

\subsubsection{Creating a new Standard file.}

To create a new Standard file the user must know what nuclides are in the standard source being used to measure the efficiency, the emission rate for photopeaks of interest and the date at which the emission rates were measured. The Create table initial dialog (see figure 7) contains a button labeled "Create standard file". A click on this button will generate the following dialog box:

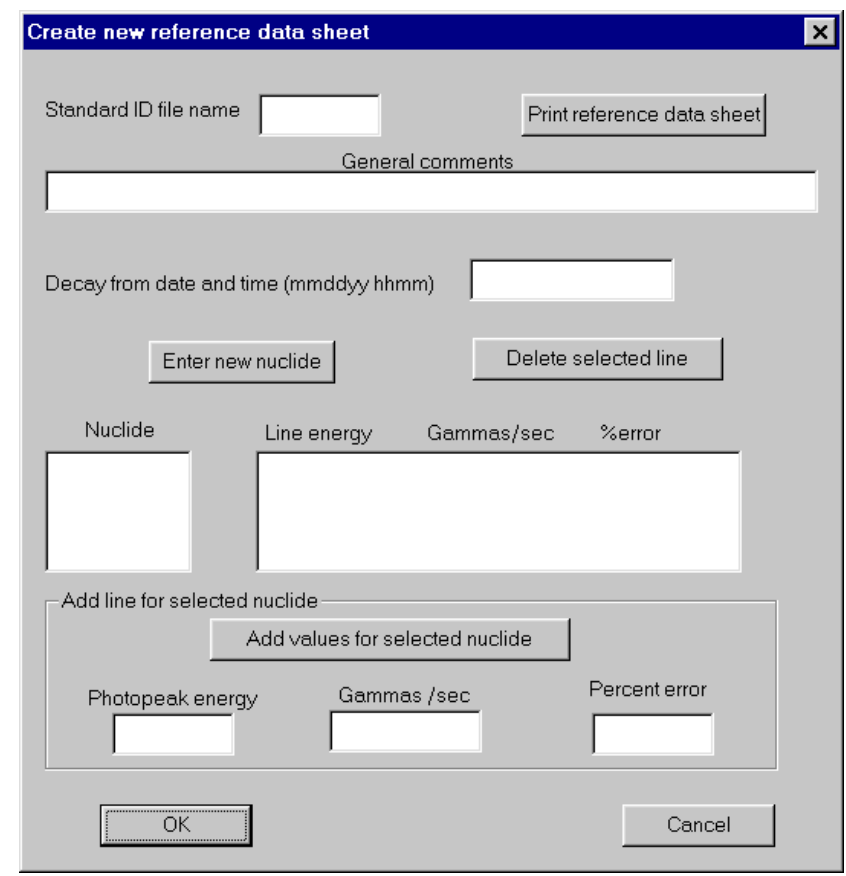

Figure 11. Create new standard reference file.

The operator must enter values for the Standard ID file name and Decay date. The ID file name can be any string of characters from which a valid file name can be constructed. The name will be used 
to create a file in PCGAP $\backslash$ DATA $\backslash E F F I C I E N C Y \backslash$ with an ".std" extension. Any string of characters can be entered in the "General Comments" box. These comments will be included in the standard file and can be used for later reference.

Values must be entered for "Decay from date and time (mmddyy hhmm)" box. This information is used to decay correct each nuclide's gammas/second value back to this date. This decay correction for each nuclide included in the standard file is based on the half-life of the nuclide found in the MASTER nuclide library found at: $\backslash$ PCGAP $\backslash D A T A \backslash L I B R A R Y \backslash N U C L I D E \backslash M A S T E R . L I B$. The format for the decay information is mmddyy, where "mm" is the month, "dd" is the day of the month and "yy" is the year. Leading zeros must be included for entries less than 2 digits. Following are some valid examples for the decay date: (1) 010300 for January 3 2000, (2) 121599 for December 151999.

The decay time format is 24 hour format "hhmm" where, "hh" is the hour and "mm" is the minute. Again leading zeros are required. The decay date must be separated from the decay time by one space. Valid examples of the complete decay date and time field are (1) 0207981200 and (2) 101497 0915.

Now that the file name and decay dates have been entered, nuclide information must be supplied. Begin by clicking on the "Enter new nuclide" button, which will cause the following dialog box to appear:

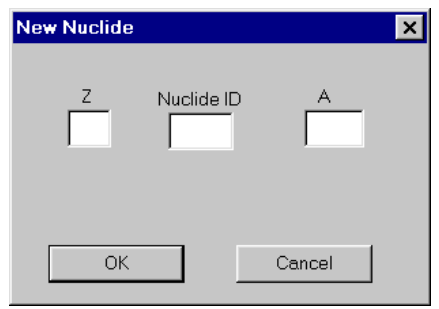

Figure 12. Enter new nuclide dialog box.

What is required is the Atomic number and weight and the chemical symbol of the nuclide to be added to the standard file. Any nuclide entered MUST be in the MASTER nuclide library. This process can be repeated until nuclides in the standard source have been entered.

As each nuclide is entered an entry will appear in the "Nuclide" list box in the "Create new reference data sheet" dialog box. Following is an example of the dialog box after two nuclides Eu-152 and $\mathrm{Sb}-125$ have been entered. 


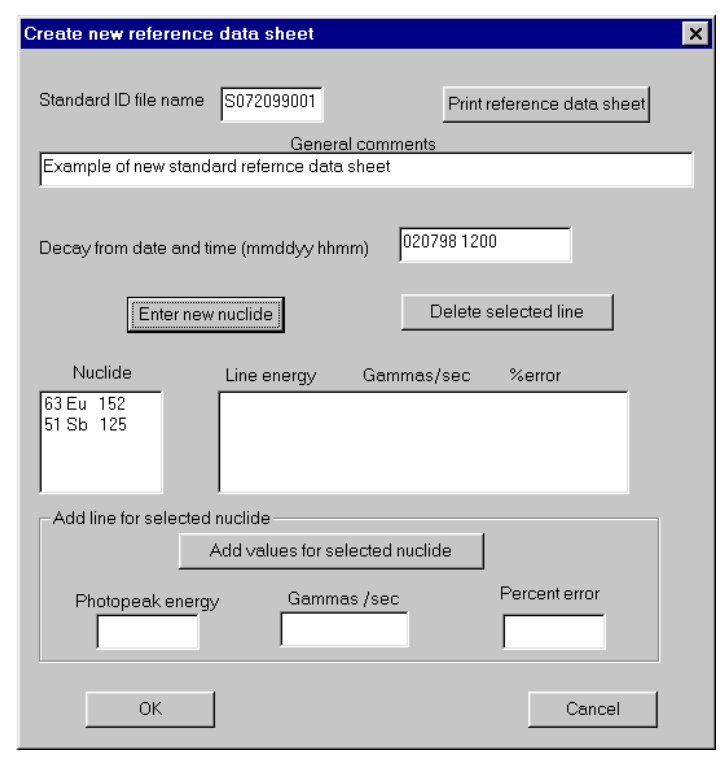

Figure 13. Example reference data info.

Once the nuclide identification information has been entered it will appear in the "Nuclide" list box. The information for the photopeaks associated with each nuclide can then be entered. Begin by selecting a nuclide, i.e. click on the nuclide entry in the "Nuclide" list box, then enter values for "Photopeak energy", "Gammas/sec" and "Percent error". Clicking on the "Add values for selected nuclide" button will add those entered values to the selected nuclide. The process of entering values for photopeak energy, Gammas/sec and percent error can be repeated for all lines associated with the nuclide. As the "Add values for selected nuclide" button is pressed the entered information will appear in the Line energy list box. Following is an example of the "Create new reference data sheet" after three photopeak values has been entered for $\mathrm{Eu} 152$.

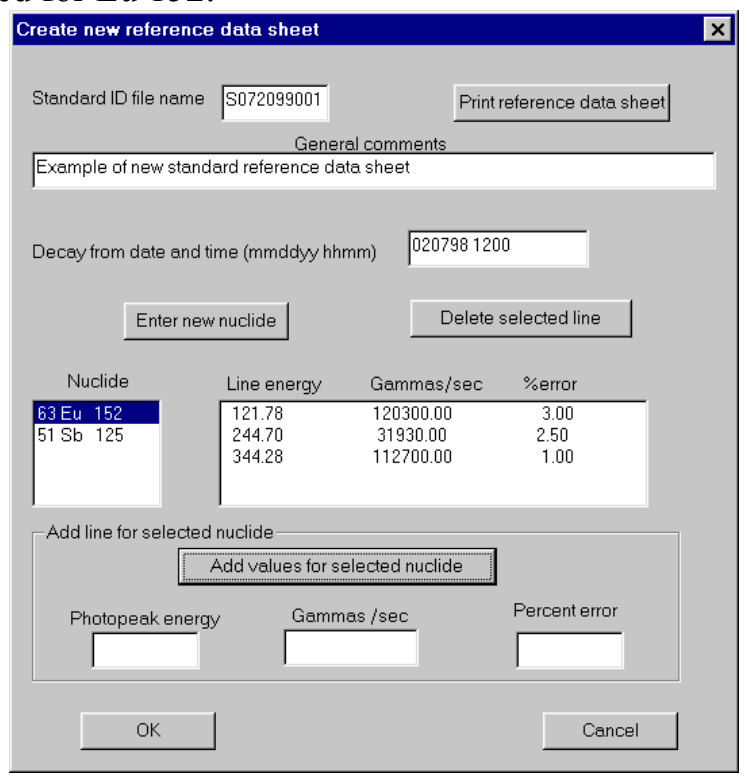

Figure 14. Example 3 lines for Eu-152.

This process is repeated until all photopeak information for all nuclides have been entered. There can be a maximum of 50 lines for all nuclides in the standard file. That means there can be two nuclides with 25 lines each or 10 nuclides with 5 lines or any combination whose total is less than 50 . 
Clicking on the "OK" button will then save the data in the file whose file name was constructed from information in the "Standard ID file name" box.

If at a later time a change is desired to be made in any of the information in the standard file, click on the "Modify exiting standard file" button in the "Enter spectrum and standard info" dialog box which is the initial dialog box for the Create table function. The "Modify existing standard file" button will read an existing standard file and bring up the "Create new reference data sheet" dialog box, where modifications can be made. The program does not allow the deletion of nuclides, but lines assigned to nuclides can be deleted and re-entered.

\subsection{Create a Nuclide Library}

One utility program NIL (Nuclide Input Library) is used to create an isotope library file that can be used by PCGAP spectrum analysis codes. Nuclide libraries in PCGAP use a structure that facilitates the lookup of an associated nuclide line from its energy. The input to the NIL program is a file that contains ASCII coded data. An example of the format for this ASCII coded data is given in appendix C. As of this version, PCGAP has no facility for generation of new ASCII code files. Included with PCGAP distribution is a file (Master.ascii) which is the NIL input file that was used to generate the Master.lib nuclide library. The entries in Master.ascii are extracted from the Brown and Firestone nuclide handbook. The user can create new libraries by either adding to the existing Master.ascii file or extracting subsets of this library to create a new file which can be used as input to NIL.

The contents of a nuclide library can be printed using the prlib.exe program.

\subsection{Spectra Conversion}

In the world of gamma ray spectrum data analysis there are as many different spectral formats as there are different analysis codes. Each spectrometer manufacturer has their own and of course PCGAP is no different. PCGAP has a utility, convert, which allows a user to select one of six different spectral file formats and convert them to PCGAP. When convert starts execution the following dialog box is displayed:

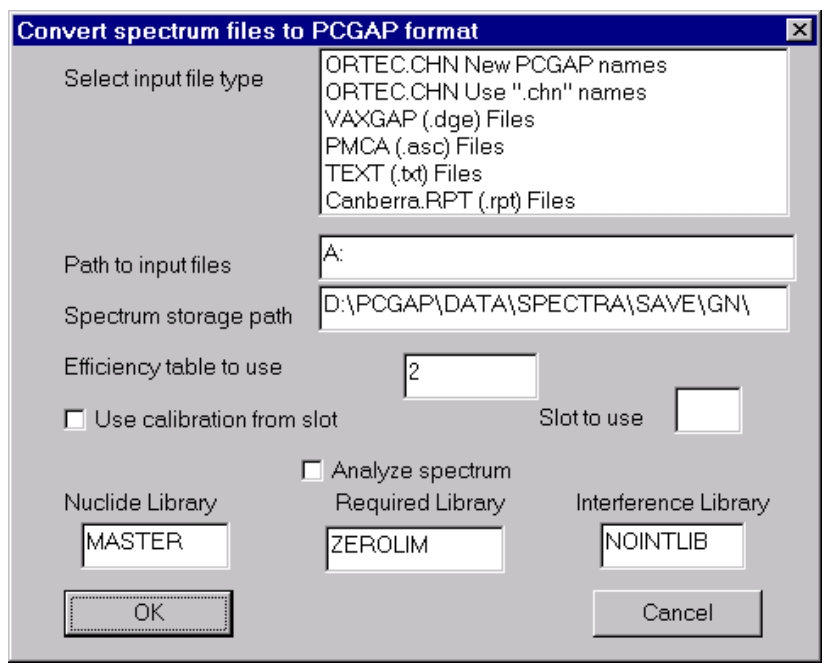

Figure 16. Convert's opening dialog. 
The operator selects the type of input file he has and enters a directory path to the location of the files to be converted. All files in the input directory will be converted. In the above example all the files on the floppy disk drive, which has the selected file type, will be converted. The spectrum storage path describes the directory path where the converted spectra will be stored. A particular efficiency table can be entered in the parameter block of all the converted spectra. Efficiency table 2 contains an efficiency of 1.0 at all energies. The energy scale from a previous calibration can be assigned to all converted spectra, by energy calibrating a spectrum in a slot file and then the convert Program can assign these energy scale parameters to each converted spectrum. Finally, the user can have the spectrum analyzed as they are converted. Information in the library edit boxes will only be used if the user checks the "Analyze spectrum" check box.

\subsection{Required Library Files and Printing Output Listings}

\section{- $\quad$ Required Library Files}

The creation of nuclide libraries is discussed in section 6.1.2 and the Interference library in section 3.5. The Required energy library is the only PCGAP library that has not been previously discussed. The required library is used to determine the activity of a radionuclide at a particular energy whether or not the photopeak in question was found by the automatic photopeak search routine. This library contains a list of photopeak energies from which the channel number of the photopeak will be determined. This energy list must be a subset of the energies defined in the nuclide library that will be used with the required energy library. Moreover, if the intensity code 1 line for a nuclide is not found by the peak search routine or it is not in the required energy library, an activity value for that nuclide will not be computed. It is always good practice to include in the required library, the intensity code 1 line energy for all radionuclides for which an analysis must be performed. Additional lines (besides the intensity code 1 line) can be included in the required library to obtain a weighted average activity value.

- $\quad$ Printing Output Files

Spectrum analysis and energy calibration produce files that are a copy of the printed results. Spectrum analysis creates/updates the files CBAG_out.lis, and CBAG_summary.lis. Spectral calibration creates/updates a Calibration.lis file. These files will normally be located in the $\backslash$ PCGAP $\backslash$ EXE directory. The utility program Print_file.exe should be used to print a copy of any of these output files. Print_file.exe uses the proper font and paper orientation to generate a standard PCGAP output listing. 


\subsection{REFERENCES}

1. E. W. Killian, L.V. East, "PCGAP: Application to analyze gamma-ray spectra on a personal computer under Windows NT", Journal of Radioanalytical Nuclear Chemistry Vol. 233. (1998)

2. J. K. Hartwell and S.G. Goodwin , "Pulser Injection with Subsequent Removal Implementation and Applications", IEEE Transaction on Nuclear Science 36 1, (February 1989)

3. E. W. Killian and T. C. Sorensen, "Concurrent Determination of Ambient Background Contribution to Low-Activity, High-Resolution Gamma-Ray Spectra,” Radioact. Radiochem.,3 (1),40,(1992).

4. Robertson, W.V. Prestwich, T.J.Kennett, “An automatic Peak Extraction Technique”, Nuclear Instruments and Methods, 100, 1972, pp. 317-324 
Appendix A

Spectral Parameter Block Structure and Definition 


\section{Appendix A - Spectral Parameter Block Structure and Definition}

The data structure used in the parameter block ( 512 bytes) of every spectrum is given below. Only the following are defined in the structure, the rest of the 512 bytes are undefined.

$\begin{array}{ll}\text { Parameter type } & \text { Mneumonic } \\ \text { Floating } & \text { Ltime } \\ \text { Floating } & \text { Rtime } \\ \text { Integer } & \text { Start_time } \\ \text { Integer } & \text { Start_date } \\ \text { Integer } & \text { S_Num } \\ \text { Integer } & \text { Collect_time } \\ \text { Integer } & \text { Collect_date } \\ \text { Floating } & \text { Collect_Duration } \\ \text { Floating } & \text { Smpl_fraction } \\ \text { Floating } & \text { Eff_mult } \\ \text { Integer } & \text { Eff_table } \\ \text { Floating } & \text { Distance } \\ \text { Floating } & \text { Volume } \\ \text { Character string }(8) & \text { Volume units } \\ \text { Integer } & \text { No_channels } \\ \text { Floating } & \text { Total } \\ \text { Floating } & \text { Sys_flow } \\ \text { Floating } & \text { Sys_volume } \\ \text { Character string }(8) & \text { Sys_volume units } \\ \text { Floating } & \text { Power } \\ \text { Floating } & \text { Coll_pos } \\ \text { Floating } & \text { Err_eff } \\ \text { Floating } & \text { Err_geo } \\ \text { Floating } & \text { Err_oth } \\ \text { Floating } & \text { Sc_sum } \\ \text { Character string }(4) & \text { Mca_system } \\ \text { Floating } & \text { Zero_offset } \\ \text { Floating } & \text { Ea_co } \\ & \\ \text { Floating } & \text { Eb_co } \\ & \\ \text { Floating } & \text { Ec_co } \\ \text { Floating } & \text { Wa_co } \\ \text { Floating } & \text { Wb_co } \\ \text { Floating array }(6) & \text { Er_mx } \\ & \\ & \end{array}$

Description

Live time in Centi-seconds

Real time in Centi-seconds

Time at start of count HHMM

Date at start of count MMDDYY

Sample number

Sample collection time HHMM

Sample collection date MMDDYY

Sample collection duration HOURS

Fraction of sample counted

Efficiency correction factor

Efficiency table number

Source to detector distance

Volume/weight of sample

Units of the volume/weight

Number of channels in spectrum

Total number of counts in spectrum

System flow rate (volume/minute)

System volume

Units of the system volume/weight

Reactor Power (Mega Watts)

Collimator position

Error in efficiency calibration

Error in source positioning

Error in other parameters

"C" term of random summing

Correction equation $\mathrm{A}+\mathrm{Bt}+\mathrm{Ct}^{2}$

Detector ID

Channel number at zero energy

"A" term of energy equation,

$\mathrm{A}+\mathrm{BX}+\mathrm{CX}$

" $\mathrm{B}$ " term of energy equation, $\mathrm{A}+\mathrm{BX}+\mathrm{CX}^{2}$

"C" $\mathrm{C}$ " term of energy equation, $\mathrm{A}+\mathrm{BX}+\mathrm{CX}^{2}$

" $A$ " term of width equation, $A+B X$

" $B$ " term of width equation, $A+B X$

Calibration error matrix 


\begin{tabular}{|c|c|c|}
\hline Floating & Sa_sum & $\begin{array}{l}\text { " } \mathrm{A} \text { " term of random summing } \\
\text { Correction equation } \mathrm{A}+\mathrm{Bt}+\mathrm{Ct}^{2}\end{array}$ \\
\hline Floating & $\mathrm{Sb} \_$sum & $\begin{array}{l}\text { " } \mathrm{B} \text { " term of random summing } \\
\text { Correction equation } \mathrm{A}+\mathrm{Bt}+\mathrm{Ct}^{2}\end{array}$ \\
\hline Integer & No_pulser & Number of INEEL pulsers used \\
\hline Floating & Pulser_rate & Rate of pulser injection, cps \\
\hline Floating & Pls_one_kev & Lower Pulser equivalent energy \\
\hline Floating & Pls_two_kev & Upper Pulser equivalent energy \\
\hline Integer & Pls_channels & Number of channels in pulser buffers \\
\hline Integer & Pls_one_start & Start channel of lower pulser buffer \\
\hline Integer & Pls_two_start & Start channel of upper pulser buffer \\
\hline Floatin & Cal_pulser_wd & Width of pulser when calibrated \\
\hline Floating & Low_pulser_wd & Width of low pulser \\
\hline
\end{tabular}

Table A-1. Spectrum Parameter Block Data Structure

Several items in the parameter block are not used by PCGAP, but space is provided for compatibility with other versions of a PCGAP type analysis. The following figure describes the parameters used in PCGAP. The variable names are those used in the parameter structure defined above.

- LTIME - The live time accumulated during the sample count

- $\quad$ RTIME - The real time accumulated during the sample count

- START_TIME - The time of day of the count in 24 hour format

- $\quad$ START_DATE - The date of the count (MMDDYY)

- COLLECT_TIME - The time at which sample was collected 24 hour format

- COLLECT_DATE - The date on which the sample was collected

- COLLECT_HOUR - The time interval during which the sample was collected. Useful for counting air filter samples.

- EFF_TABLE - A four digit number which is used in conjunction with the DISTANCE parameter value to determine which detector efficiency table (file) to use.

- DISTANCE - A four digit number indicating the source to detector distance. This parameter is used in conjunction with efficiency table number to determine the efficiency table to use.

- VOLUME - The volume/weight of the sample is entered here.

- VOLUME_UNITS - The units of the volume.

- NO_CHANNELS - The number of channels in the spectrum

- MCA_SYSTEM - Two character designation of the detector system

- ZERO_OFFSET, EA_CO, EB_CO, EC_CO - Energy equation coefficients

- WA_CO, WB_CO - Width equation coefficients

- SA_CO,SB_CO, SC_CO - Random summing correction equation coefficients.

- NO_PULSER - Integer indicating whether INEEL pulser is installed on multichannel analyzer. $0=$ no pulser $2=$ two peak pulser installed

- PULSER_RATE - Rate at which pulser system is injecting pulses. Counts/sec.

- PLS_ONE_KEV - Low amplitude pulser equivalent energy.

- PLS_TWO_KEY - High amplitude pulser equivalent energy.

- PLS_CHANNELS - Number of channels allocated to pulser buffer.

- PLS_ONE_START - Starting channel of low pulser buffer

- PLS_TWO_START - Starting channel of high pulser buffer.

- PLS_ONE_PS - Low pulser offset (in channels) 
- PLS_TWO_PS - High pulser offset (in channels)

- CAL_PULSER_WD - Width of low pulser at calibration

- LOW_PULSER_WD - Current width of low pulser. 
Appendix B

\section{Flag Values Printed on PCGAP Output}




\section{Appendix B - Flag Values Printed on PCGAP Output}

The integer value printed in the FLAGS column of the PCGAP output file indicates the operations performed on the photopeak during the analysis. Figure B-1 indicates the basic values. The actual printed flag value will be a sum of the appropriate basic values. As an example a flag value of 554 $(=2+8+32+512)$ indicates the following:

1. The Initial fit was performed the nonlinear fitting process as part of a multiplet.

2. The result was corrected for ambient background.

3. The result was interference decontaminated.

4. The result was rejected as an outlier and was not used in the final weighted average.

\begin{tabular}{c} 
Flag Value \\
\hline 0 \\
1 \\
2 \\
3 \\
8 \\
32 \\
128 \\
512
\end{tabular}

Table B-1. Flag value definitions

Nonlinear fit result for a singlet
Linear fit result for a singlet
Nonlinear fit result part of a multiplet
Linear fit result for part of a multiplet
Ambient background corrected
Interference decontaminated
Listed as a clean line in the interference library
Result was an outlier and not used in weighted average


Appendix C

Nuclide Library ASCII Input Format 


\section{Appendix C - Nuclide Library ASCII Input Format}

An example of the data format that is used as input to NIL to generate nuclide libraries for PCGAP follows:

24,CR, 51, 320.084198, 2.770600E+01D, 9.85000E+00, 1 25,MN, 56,2657.581055, 2.578500E+00H, 6.53000E-01, $26, \mathrm{FE}, 59,192.348999,4.451000 \mathrm{E}+01 \mathrm{D}, 2.95000 \mathrm{E}+00,2$ 26,FE, 59,1099.250977, 4.451000E+01D, 5.61000E $+01,1$

All values are comma delimited with each field having the following contents:

Atomic weight, Chemical symbol, Atomic number, Photopeak energy, Nuclide half life, Branching ratio, Intensity code.

The Chemical symbol can be up to 4 characters long. The half life value must be terminated with a character which indicates the units of the half life:

- $\mathrm{S}=$ Seconds

- $\mathrm{M}=$ Minutes

- $\mathrm{H}=$ Hours

- $\mathrm{D}=$ Day

- $\mathrm{Y}=\mathrm{Year}$

The branching ratio is expressed as a percentage. The intensity code is an integer between 1-9 with only one line in the nuclide set identified with a intensity code of 1 . 
Appendix D

Users Guide for the Interactive Peak Fitting Program GINA 


\section{Appendix D - Users Guide for the Interactive Peak Fitting Program GINA}

\section{D-1.0 Introduction}

The interactive photopeak fitting program, GINA, was initially written to run on a VAX/VMS platform. It used the X-Windows graphical interface, and required the use of a X-Window terminal. Two years ago the code was ported to a Personnel Computer (PC) platform running Windows NT. It retained the use of the X-Window interface and required the use of a X-Windows server for Microsoft windows. The X-Window software was obtained from a third party and was difficult to install and keep current with the never ending releases of new versions. Consequently GINA has been rewritten to replace the $\mathrm{X}$-Window calls with native Window graphic functions. This document describes how to use this new Windows version of GINA.

Please note, this program is an integral part of the Gamma-ray pulse height analysis system, PCGAP $^{\odot}$. It can only use PCGAP formatted spectral files. 


\section{D-2.0 Starting GINA}

GINA is distributed with the PCGAP software. The executable for the code resides in the $\triangle P C G A P \backslash E X E$ directory. When the PCGAP software is installed, a "Short Cut" to this file was created. The user can start the code running by clicking on the short cut or double clicking on the executable from Windows Explorer. The window depicted in figure D-1 appears when GINA starts execution. Initially, there is only one menu item displayed. It is labeled "Control".

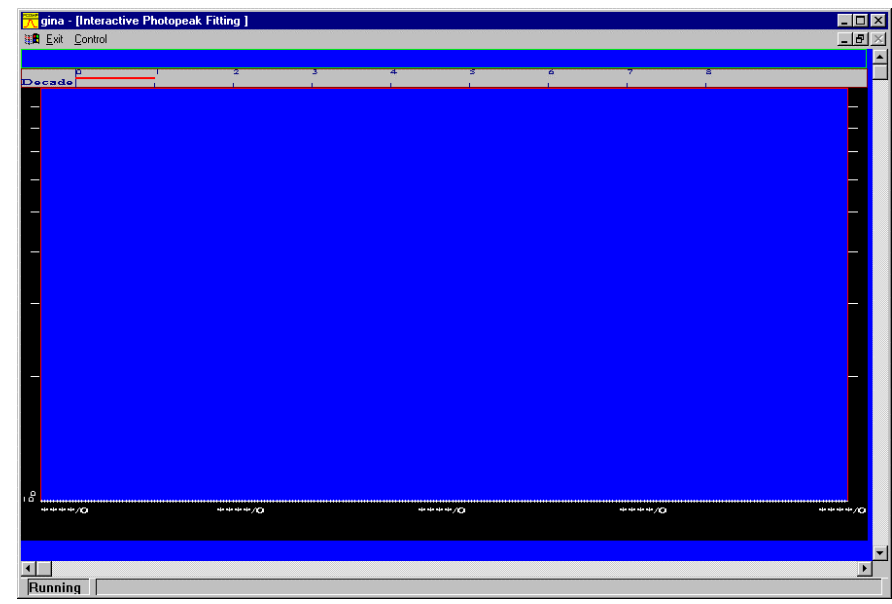

Figure D-1. GINA starting window

Clicking on the "Control" menu will generate a pull-down menu, which initially will have only two active items:

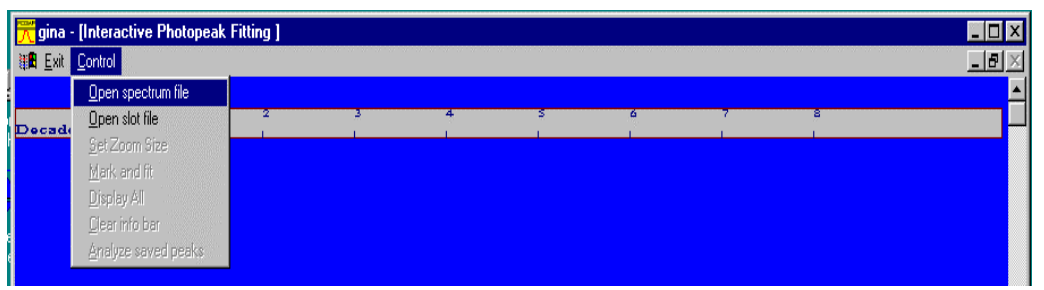

Figure D-2. Initial control menu items

The "Open spectrum file" and "Open slot file" functions allow a spectrum file to be opened and the spectrum displayed. When the spectrum file has been opened, the other menu items under control will become active. A discussion of these other items will follow in subsequent sections.

Both the open functions, when selected, will create a standard Windows open file dialog. The "Open spectrum file" will initially open a window to the "pcgapldatalspectralsavelgn" directory. The user can, of course, move to any other directory where there are valid PCGAP spectrum files. The "Open slot file" initial directory will be "pcgapldatalslot" where valid slot files can be opened. Valid slot files have extension ".dat". As was the case with the "Open spectrum file", the user can use this dialog to move to any directory where there are valid PCGAP spectrum files (slot files or normal spectrum files). An example of the "Open spectrum file" dialog is given below: 


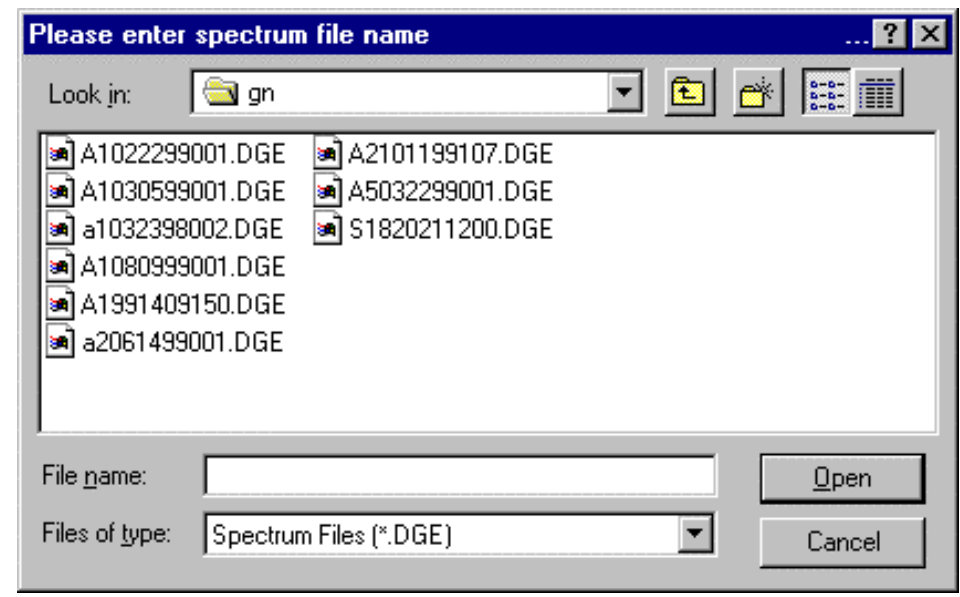

Figure D-3. Open spectrum file dialog

To open the spectrum file, click on the file name and then click the "open" button. When the spectrum file has been selected, the main GINA window will display the spectral data.

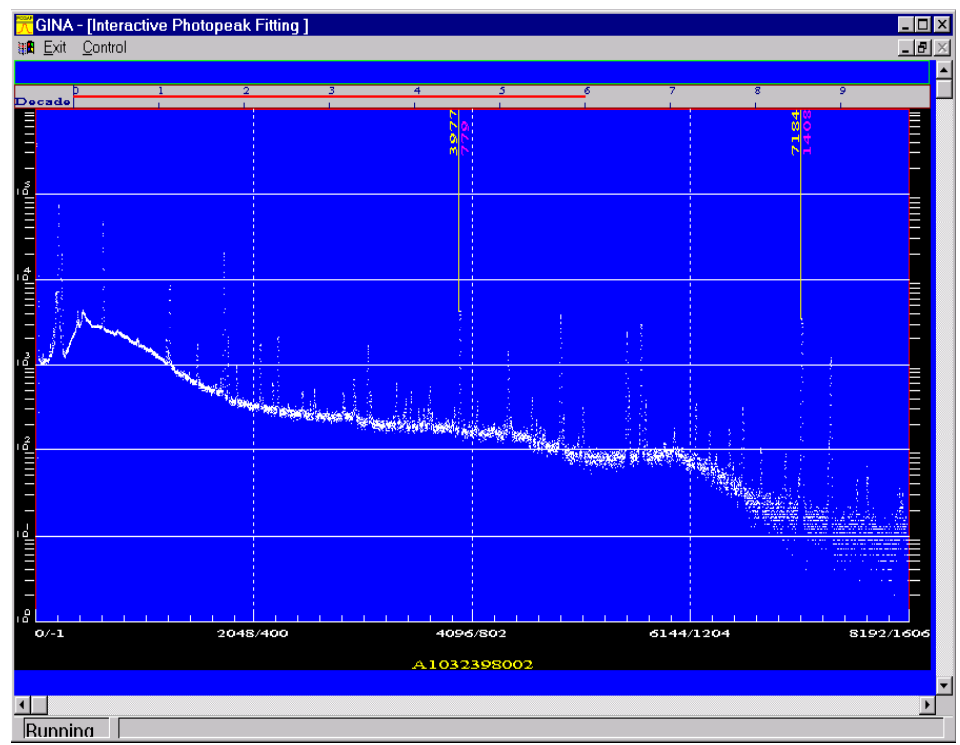

Figure D-4. Gina spectrum display

The spectral data is displayed on a semi-log scale; the vertical axis is $\log _{10}$ and the horizontal axis is channel number. The main GINA window has several items displayed and certain clicks inside certain regions of the window can cause spectral data to be displayed differently. A discussion of these various items are given below:

\section{D-2.1 Decade Bar}

The decade bar is a red line in a thin rectangle with a gray background. Across the top of the rectangle are integers from 0 to 9 . The integer represents the number of decades displayed on the vertical axis. The user can control the starting decade of the display by clicking on the left side of the bar and the maximum number of decades by clicking to the right end of the bar. The red decade line will start at the 
beginning decade and end at the maximum. Following is an example where the number of decades for the spectrum shown in figure D-4 has been adjusted to display 2 decades only.

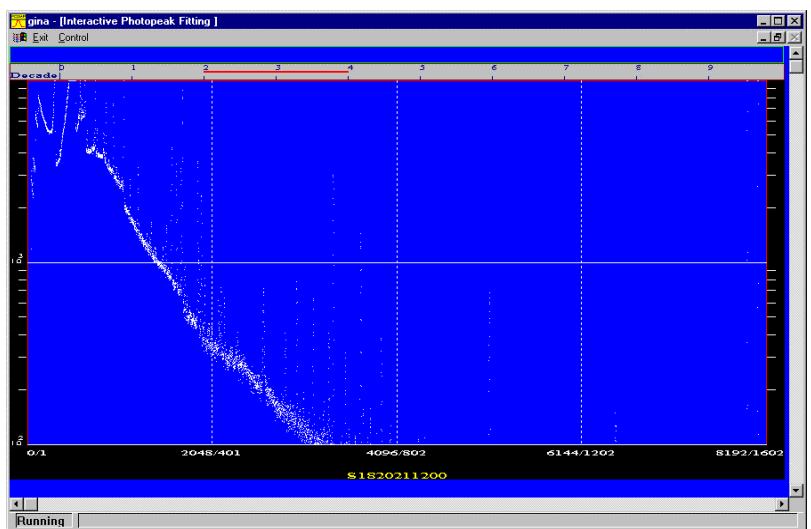

Figure D-5. Spectral display 2 decades

The spectral data is being displayed starting at $10^{2}$ with a maximum of $10^{4}$. This vertical control of the display can be very useful for examining weak peaks in the presence of large peaks.

\section{D-2.2 Informational Display}

Across the bottom of the X-axis are a series of five evenly spaced numbers, with a dotted line drawn to the top of the spectral display. The series of numbers are two integer values separated with "/". The first number is the channel number where the dotted line is drawn and the second number is the energy at the channel number.

Below the channel and energy scale numbers is the spectrum file name which was opened to display the data.

A right click on the spectral display will cause the channel number closest to the cursor and its associated energy to be displayed in the information region. Following is an example where three mouse clicks were made on the spectrum at channels 3977 and 7184 : 


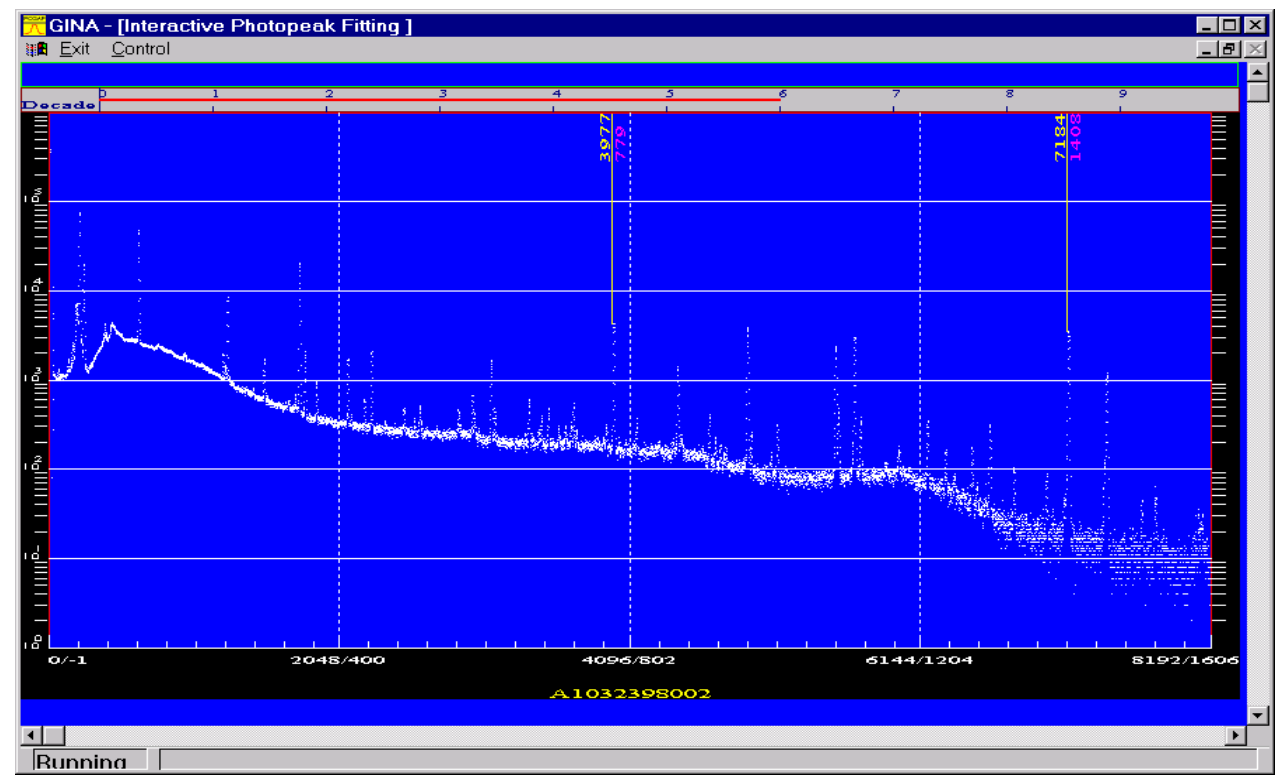

Figure D-6. Right click in spectral area

There is no limit to the number of right clicks that can be made in any one spectrum. After a while the information displayed overlaps with a previous click and every thing becomes unreadable. For this reason there is a "Clear Info Bar" function under the "Control" menu. This function will clear all lines in the spectral data region.

\section{D-2.3 Number of Channels Displayed}

A mouse click inside spectral display area, will cause the display to "zoom" into a region centered on the channel closest to where the mouse click was made. The total number of channels displayed in the zoom mode is controlled by the "Set zoom size" function under the "Control" menu item. Below is an example of the spectrum display after a click in the spectrum region. 


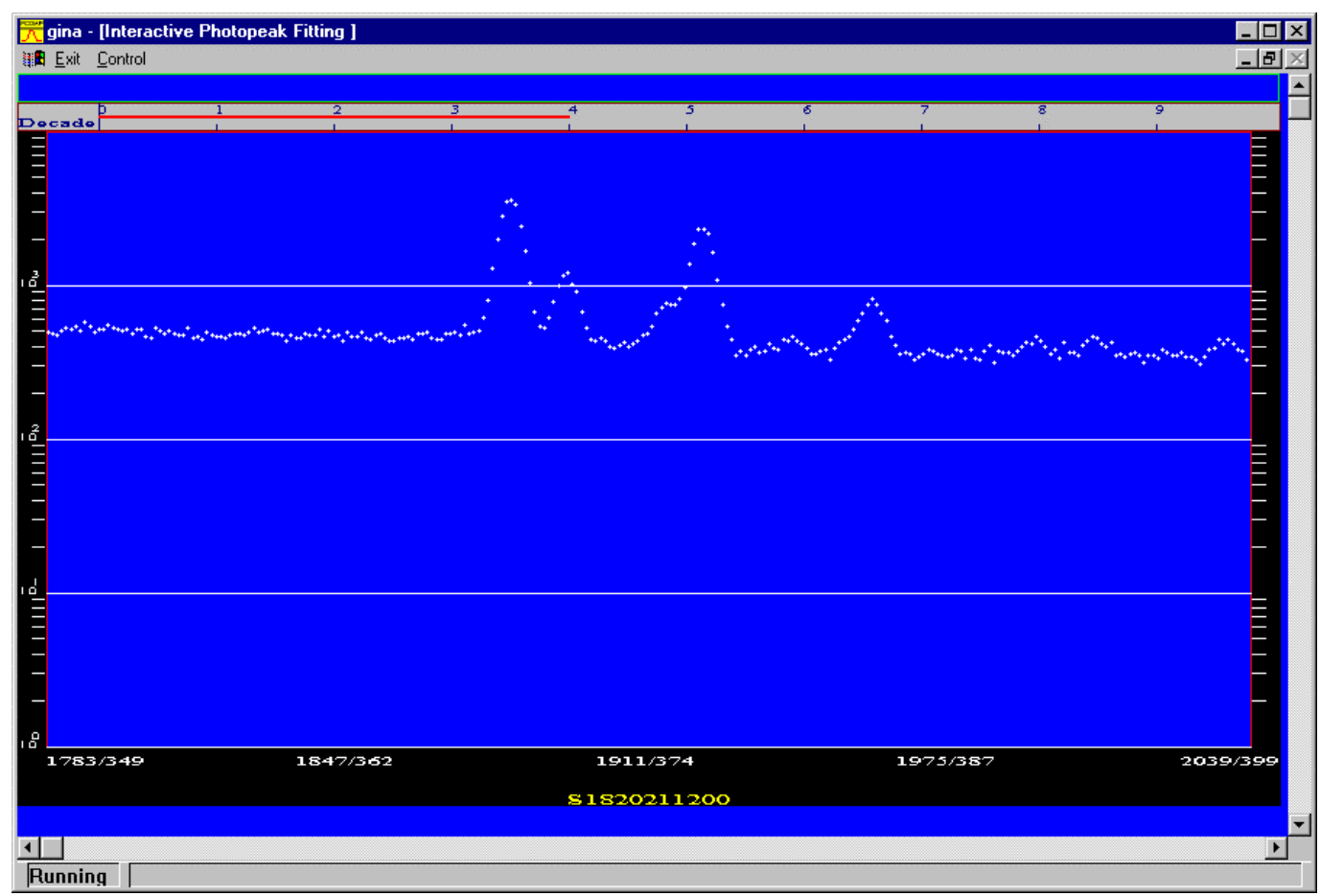

Figure D-7. Spectrum “zoom” function

It should be noted that a right click in a spectrum which is zoomed around a channel number is a valid function. 


\section{D-3.0 "Control" Menu Items}

We have discussed two of the menu items, "Open spectrum file" and "Open slot file" previously. The following sections describe the other menu functions which are part of the "Control" pull-down menu which is shown in the following figure.

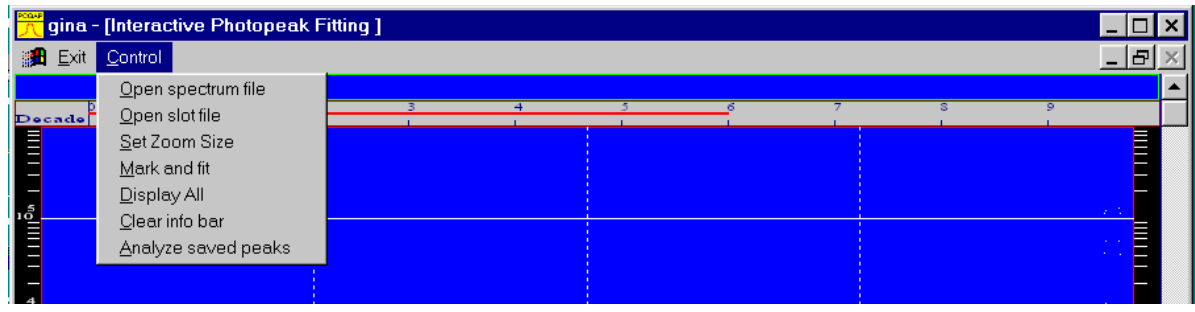

Figure D-8. Control pull down menu

- $\quad$ Set Zoom Size

This functions controls the number of channels displayed when a region of the spectrum is "zoomed" into. A left click in the spectral display will zoom the spectrum. The following dialog will appear when the Set zoom size function is selected:

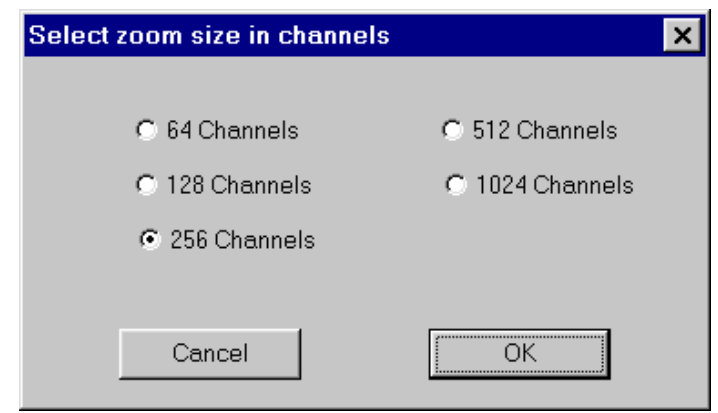

Figure D-9. Set zoom dialog

The operator can choose any one of the zoom sizes by clicking on the radio button corresponding to the desired number of channels.

- Display all

This function will display the complete spectrum. It has the opposite effect of the zoom function.

There is no additional input required for this function.

- Clear Info bar

This function will clear the channel and energy values and their associated notation lines. See section above There is no additional input required for this function.

- Analyze saved peaks

GINA has the capability to save the results of a fit to a photopeak. If this function is active, the saved fit results will be used as input to the PCGAP routines which assign nuclide identifications to the 
saved photopeaks and then proceeds to determine concentration values of the assigned nuclides. The nuclide library used to make the nuclide assignments is stored in the "pcgapldatallibrarylisotope gina.lib" library file. This file can be modified and changed to reflect user needs in the same manner as other PCGAP nuclide libraries. For the analysis to function correctly, the spectrum file must have suitable values in its Parameter block such as; efficiency table numbers, real and live times and sampling times necessary to compute decay corrections.

This function is an on-off toggle. If it is on it will be checked. Clicking on it again will clear it and remove the check mark. If the function is not selected when the exit menu item is selected the analysis will not be performed.

- Mark and fit

This function is the heart and soul of the GINA program. This function allows the user to mark fit limits, background areas and initiate fits. Because this function is used to mark channels and initiate fits it can only operate on reduced region of the spectrum. To select a region, move the cursor to the desired channel and left click. The display of the spectrum will now zoom into the region. The Number of channels displayed is a function of the zoom size. If a region of the spectrum has been selected and the "Mark and fit" function is selected the following window will be generated.

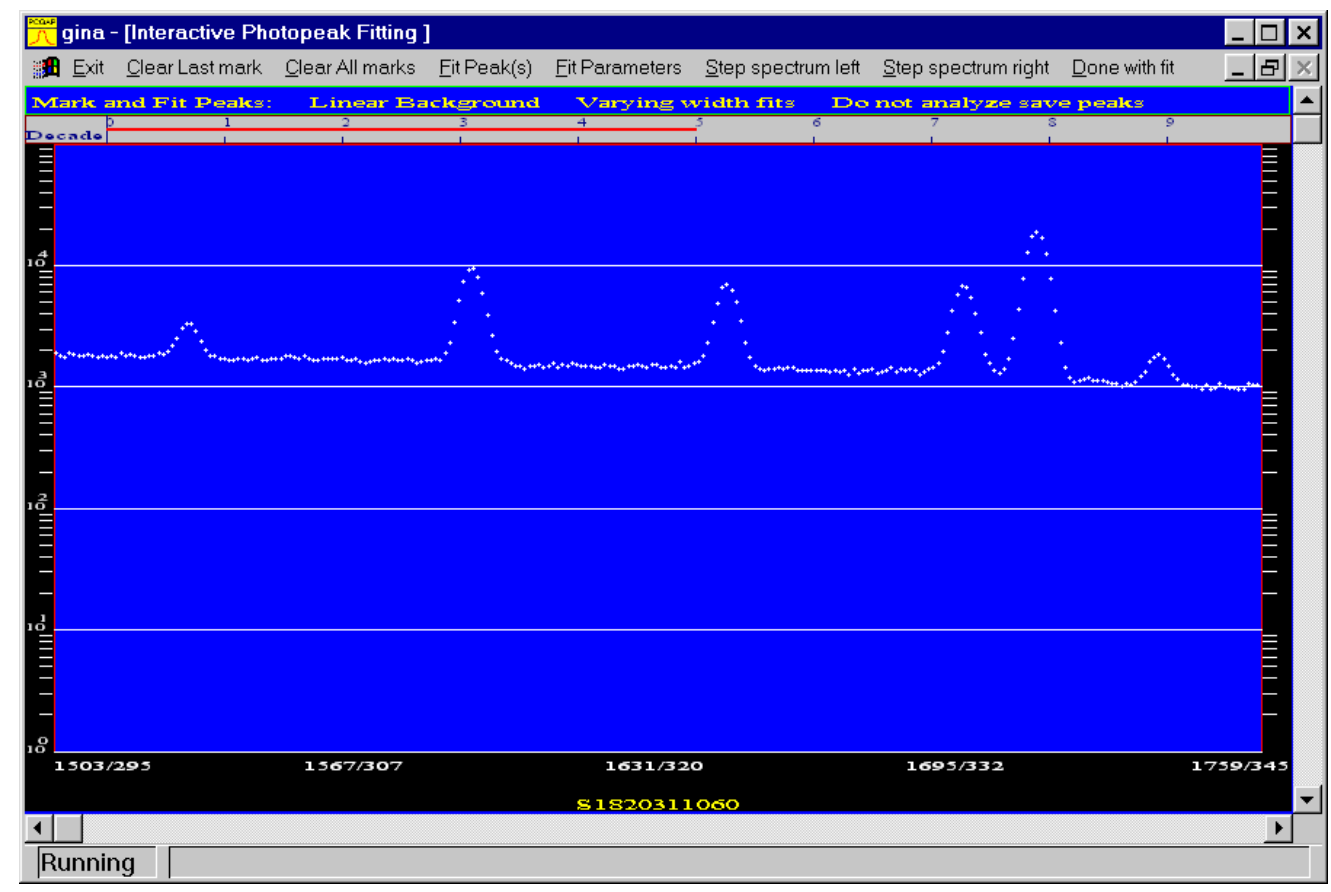

Figure D-10. Mark and fit Window

\section{D-3.1 Marking a Peak for a Fit}

This window looks a lot like the main GINA starting window, with two exceptions.

1. A new set of menu items is now being displayed.

2. A set of fit parameter values is displayed in the info window. 
The function of "marking and fitting" entails marking (with the mouse) a region around a photopeak. An iterative non-linear least squares fit of a Gaussian function to the properly marked peak can then be performed. In all cases the centroid and height of the peak is allowed to vary to minimize the residuals. The peak width is under operator control; it can be allowed to vary or remain fixed at the initial value. The spectral background under the peak is selected by the operator. The background is not part of the fit and can have two different shapes; a linear line drawn between two points or step function which roles over at the peak centroid.

The mouse buttons are used to select a peak to be fit. GINA assumes a two button mouse is being used, consequently, the left mouse button serves two functions, to mark both the left and right background regions. The examples shown in this document are gray scale and do not indicate the colors of the various peak markers. The background markers are red, the fit limit markers are yellow and the peak centroid are black. The background markers are left and right square brackets "[" and "]". In the following example the background region on the low energy side of the peak has been marked.

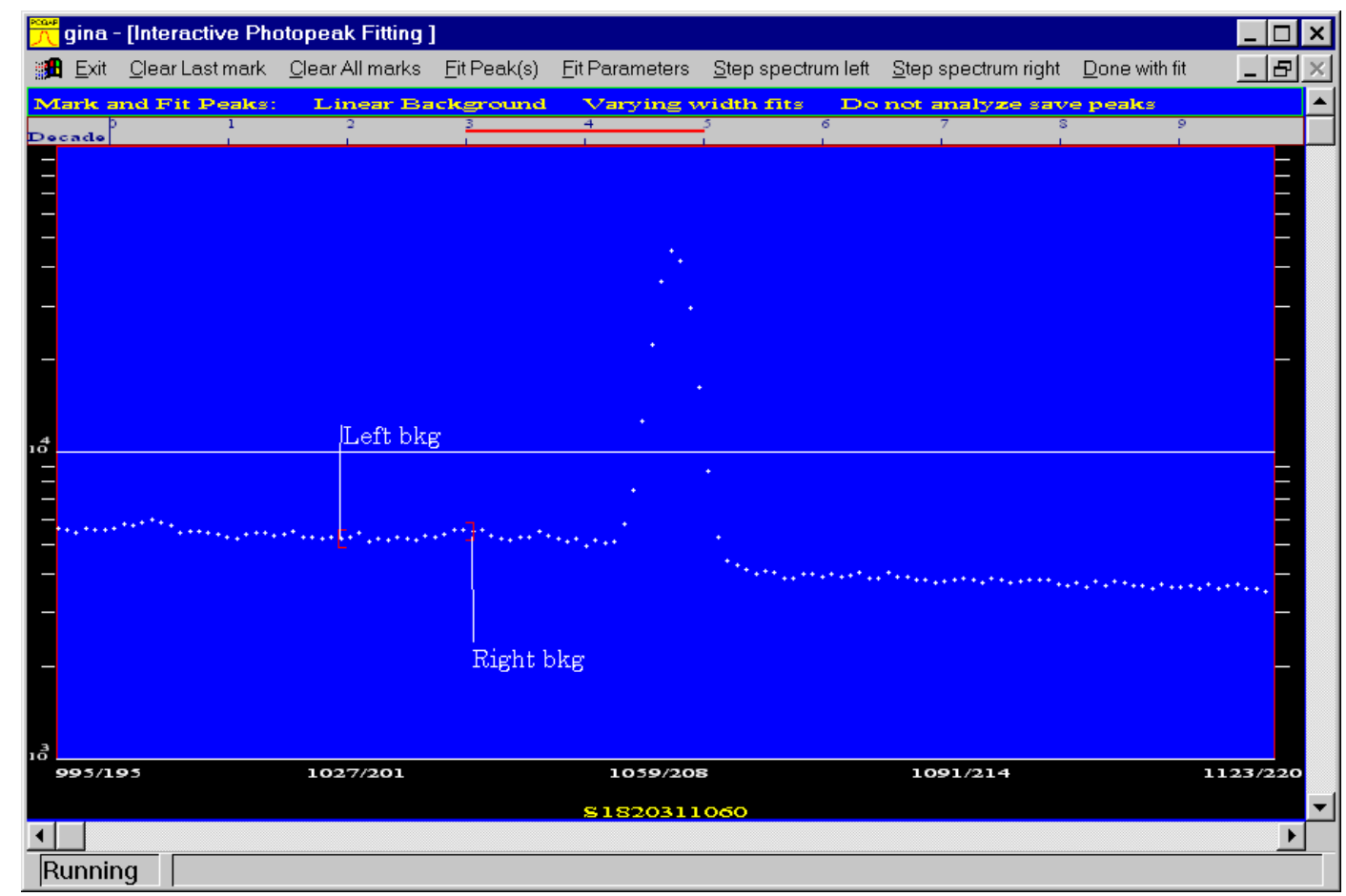

Figure D-11. Left background region

The "Left bkg" and "Right bkg" have been inserted into the example for illustrative purposes; they are NOT part of the GINA display. The square brackets are all that will appear. The position of the brackets was set by moving the mouse cursor to the desired channel and pressing the left mouse button. In this mode the first press of the left mouse button will generate a left square bracket "[",moving the mouse to new position and clicking the left mouse button again will generate a right square bracket "]". The center channel, $X_{L}$, in the region defined by the brackets will be the channel value for the background on the left side. The average number of counts in the region, $\mathrm{Y}_{\mathrm{L}}$, will be as the content value for the left background. The left background will be assigned the value $\left(\mathrm{X}_{\mathrm{L}}, \mathrm{Y}_{\mathrm{L}}\right)$. Using the left mouse button on the right side of the peak in the same manner as for the left, a right background region can be defined, from which a right side ordered pair $\left(X_{R}, Y_{R}\right)$ can be computed. For a linear line representation of the background, the line will be drawn from $\left(\mathrm{X}_{\mathrm{L}}, \mathrm{Y}_{\mathrm{L}}\right)$ to $\left(\mathrm{X}_{\mathrm{R}}, \mathrm{Y}_{\mathrm{R}}\right)$. 
Usually the set up for a fit is made from left to right; that is mark the left background then the left fit region. The delimitating marks for fit limits are ellipses " $\cup$ ". The following example illustrates the left fit mark on a peak (the left and right annotations are not part of GINA display and are added for clarification only):

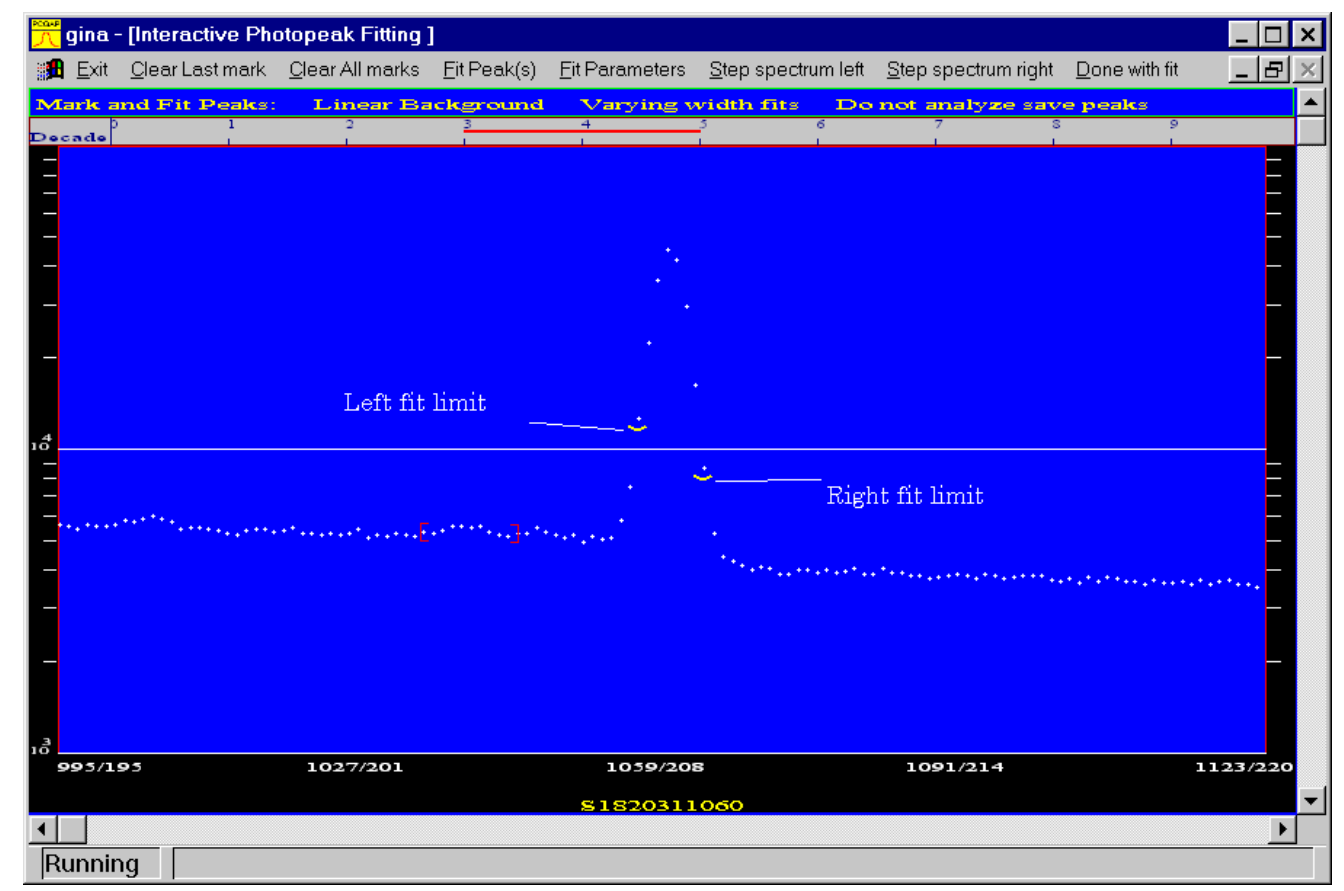

Figure D-12. Fit limits

The fit limits are set by moving the cursor to the desired channel and pressing the right mouse button. The channels between the left and right fit limit are the channels which are included in the least squares fit. If multiple peaks are included in a single fit, the left fit limit must be on the left side of the left most peak and on the right side of the right most peak. For example: 


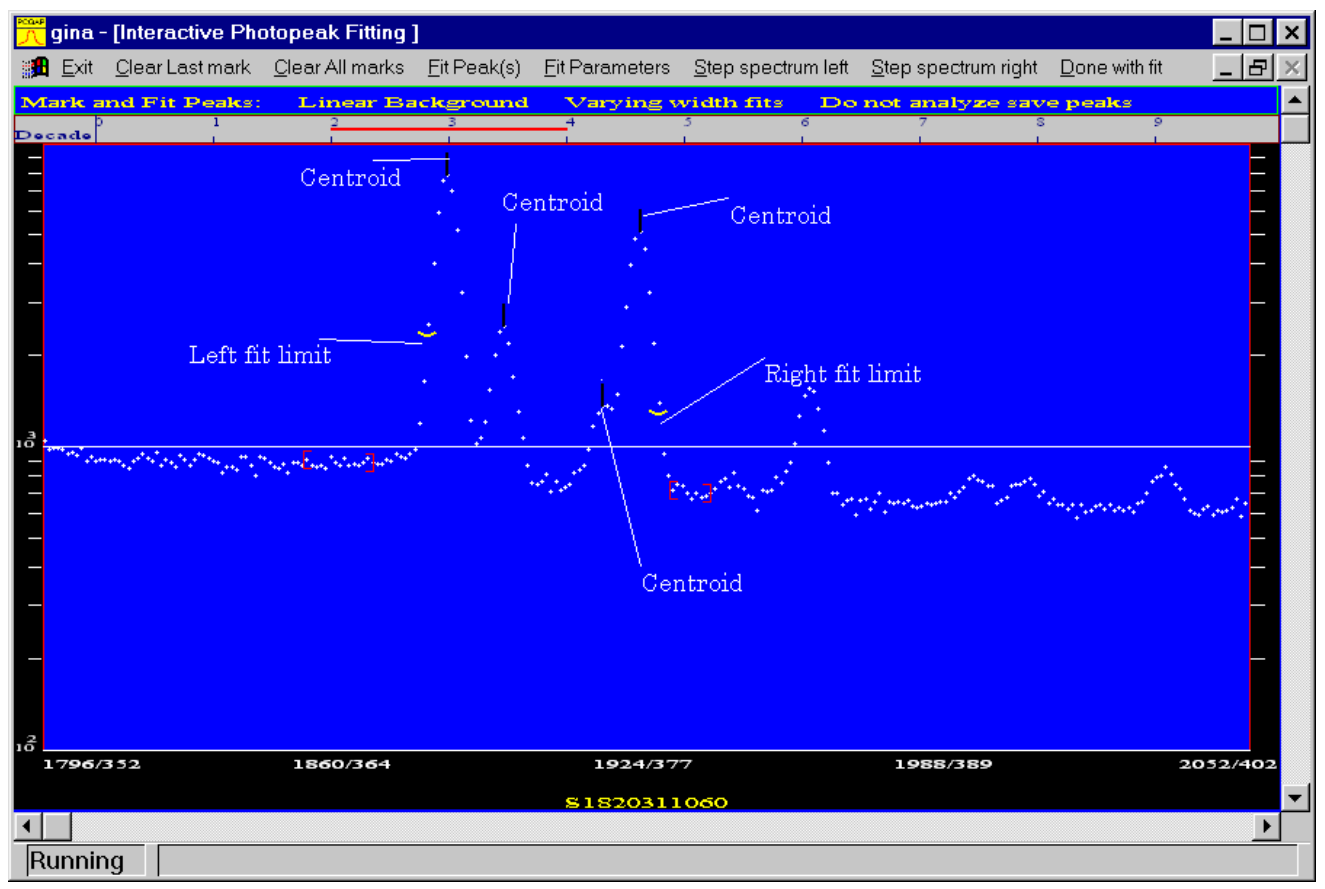

Figure D-13. Multiple fit with Centroids marked

In the above example a multiple peak fit has been set up. The centroid of each of the peaks to be included must be marked. They are marked by pressing the left mouse button with the Ctrl key.

Once all the background regions have been defined, the fit limits set and the centroids marked, a fit can be initiated by clicking on the "Fit peak(s)" menu item. At the conclusion of the fitting process the following display will be appear: 


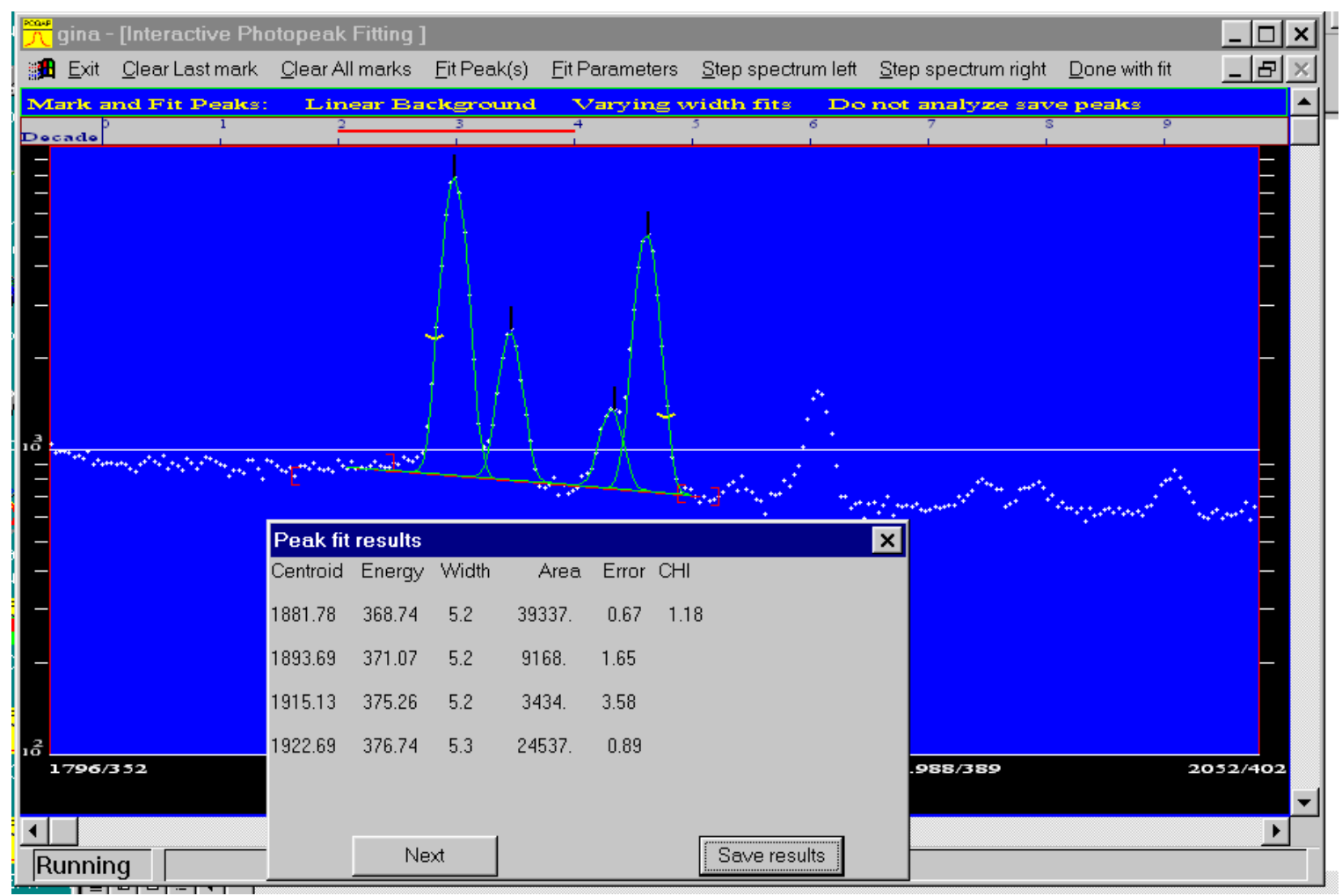

Figure D-14. Four peaks fit and results displayed

From the above example, one can see that the resulting individual component Gaussian functions are plotted (on a color monitor they are green lines). The background line is drawn in red; however, the tails of the individual Gaussian functions can overlay the background line and turn it green. Together with a visual display of the fitting function there is a dialog box which appears and displays the numeric results of the fit. There will be a line of information for each fit peak. The width values are in channel, the area error is in percent, and the CHI value is the reduced chi value calculated over the fit interval.

The user has two options, when the results of the fit are displayed. The results can be saved or ignored. If the results are saved they will be reduced to hardcopy when the Exit button is clicked. GINA also has the ability to perform a nuclide analysis on fit results which are saved, See section D-3.0 "Analyze saved peaks". Clicking on "Next" or "Save results" will dismiss the output dialog. The "Mark and Fit" window will still display the fit results. A new fit can now be set up.

Following is a description of the "Mark and Fit" menu items.

- Clear last mark

Because the mouse cursor is not always the best pointing tool, the positioning of the mark for a peak fit will some times be placed at the wrong channel. In this case the "Clear last mark" is used. It will NOT clear any other marks made earlier. It will clear a single mark only. 
- Clear All marks

Execution of this function will clear all marks and any fit results that are displayed.

- Fit Parameters

This menu item is a pull-down list of various parameters the user can modify to control the peak fitting process. An example of the pull down menu follows:

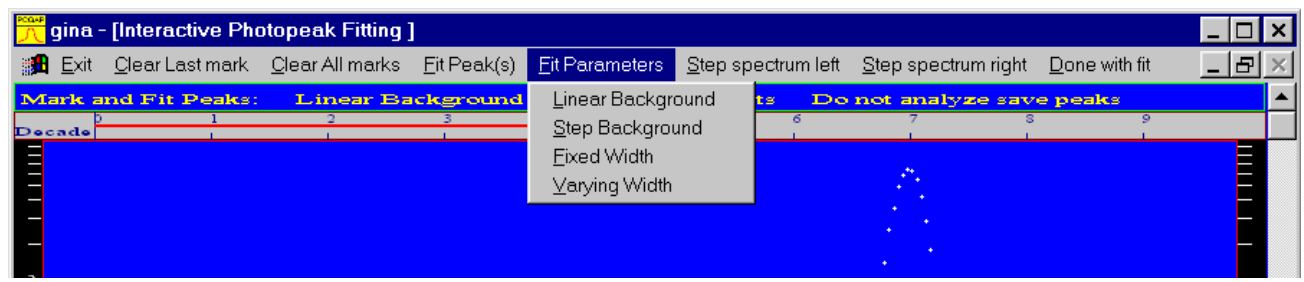

Figure D-15. Fit Parameters pull-down menu

- $\quad$ Linear Background

Selection of this option will use the linear background model for determining the background under a photopeak. An example of using this model was shown in figure D-14.

- $\quad$ Step Background

This function selects a step background model for photopeak background. An example of a fit using this model is shown below:

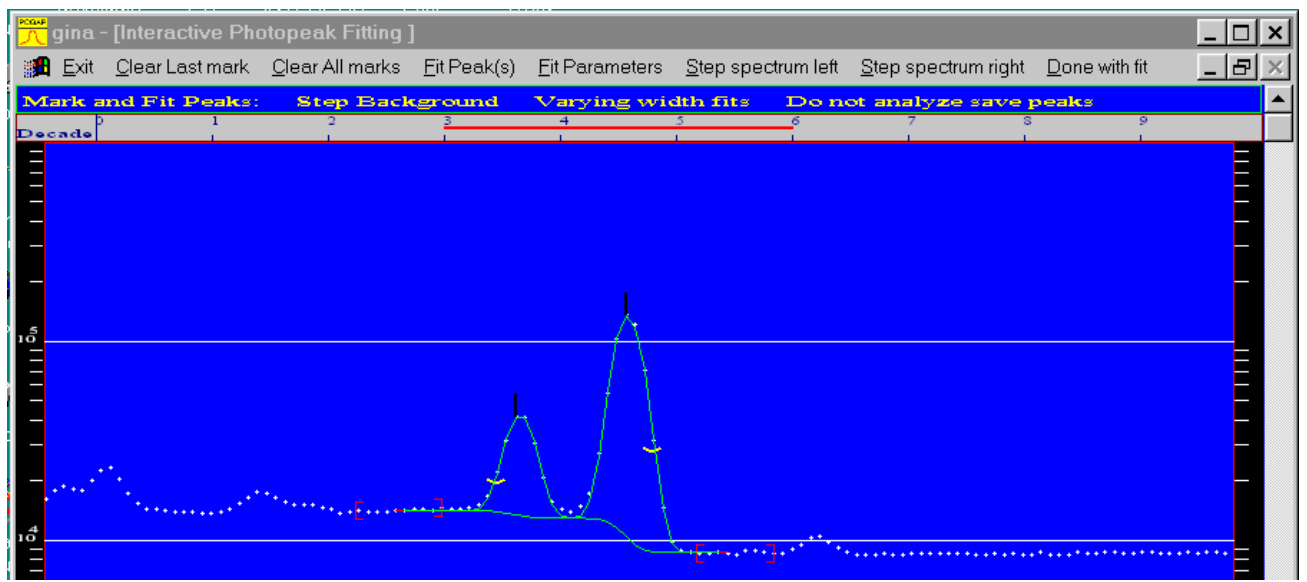

Figure D-16. Step background model

In the example in Figure D-16 the step the background takes at the centroid is clear. The step function will yield a result that approximates the linear background model if the background regions on both sides of the peak is nearly flat. 
- $\quad$ Fixed Width

The peak width will be set to an initial value and held fixed during the fitting process.

\section{- $\quad$ Varying Width}

The peak width will be set to an initial value and allowed to vary during the fitting process.

- $\quad$ Step spectrum left

A click on this menu item will step the spectrum to the left the number of channels currently in the window. This function permits the positioning of the spectrum display to a new region, with out having to return to the main display window.

- Step spectrum right

This function steps the spectral display to the right.

- Done with fit

This function returns the display back to the main display window. It does NOT terminate the program. 
Appendix E

Users Guide for the Spectral Manipulation Program PANDA 


\section{Appendix E - Users Guide for the Spectral Manipulation Program PANDA \\ E-1.0 Introduction}

The PANDA program is a major component of the PCGAP gamma-ray pulse height spectrum analysis package which runs on the Windows NT operating system. PANDA provides the user with the following major functions:

- Spectral Display

- Control of Canberra Acquisition Interface Module, AIM, for the collection of pulse-height gammaray data.

- Spectral analysis

- Spectrum energy scale determination

The PANDA program can be found in the $\backslash$ PCGAP $\backslash$ EXE folder and begins execution by displaying the following window:

Figure E-1. PANDA Startup Display

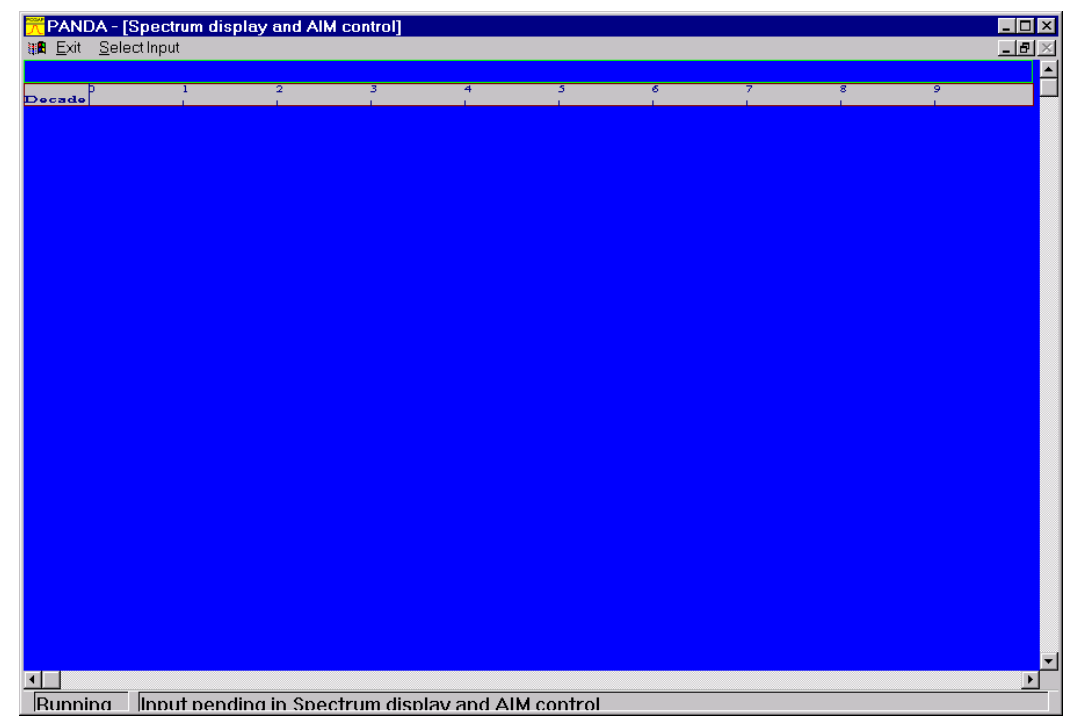

The following sections will describe how to use the various menu items and associated functions. Menu items will appear and disappear as various functions are exercised. 


\section{E-2.0 Exit and Select Input Menus}

This beginning window contains two menu items: "Exit" and "Select Input". A click on the Exit menu will cause PANDA to terminate. Selection of the "Select Input" menu will generate a pull down menu with the following functions displayed:

Figure E-2. Select Input function items

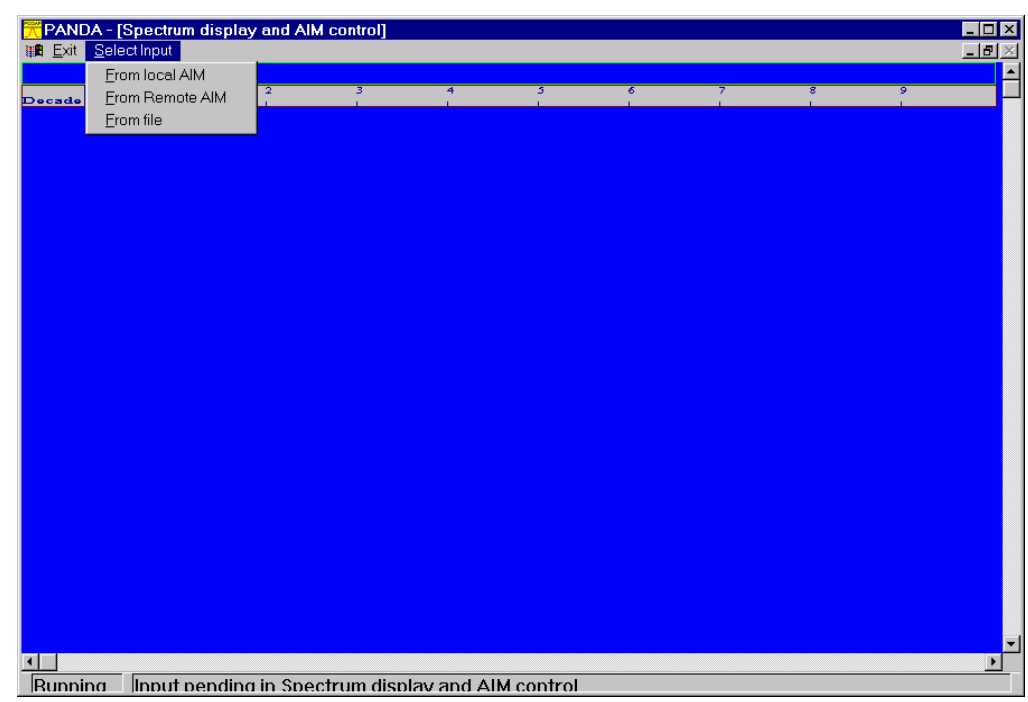

The select input menu allows the user to select where the source of data is going to come from. Following is a discussion of each of the data sources.

\section{- $\quad$ From Local AIM}

In order for this function to work the Canberra Genie-2000 programming library, Model S560 and the AIM Ethernet protocol must be installed on the computer. One or more detector systems must be loaded in the Genie-2000 database (See Canberra's MCA Input Definition Editor, MID). If these conditions are met then the following dialog box will appear:

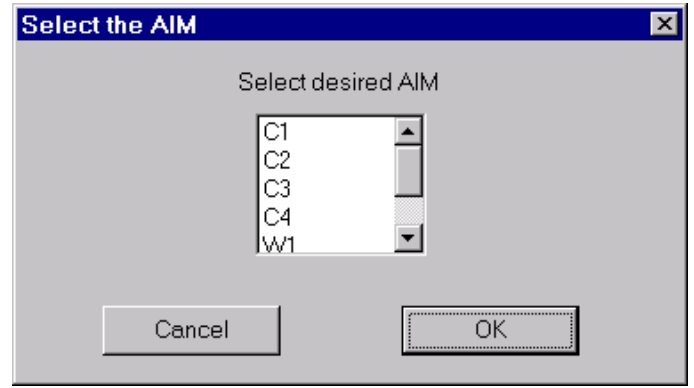

Figure E-3. Select Local AIM detector

The detector systems, which have been loaded into the database, will appear in the list box. Click on any one of the detector systems and click the OK button; the spectrum from the AIM will now be displayed. For Example: 


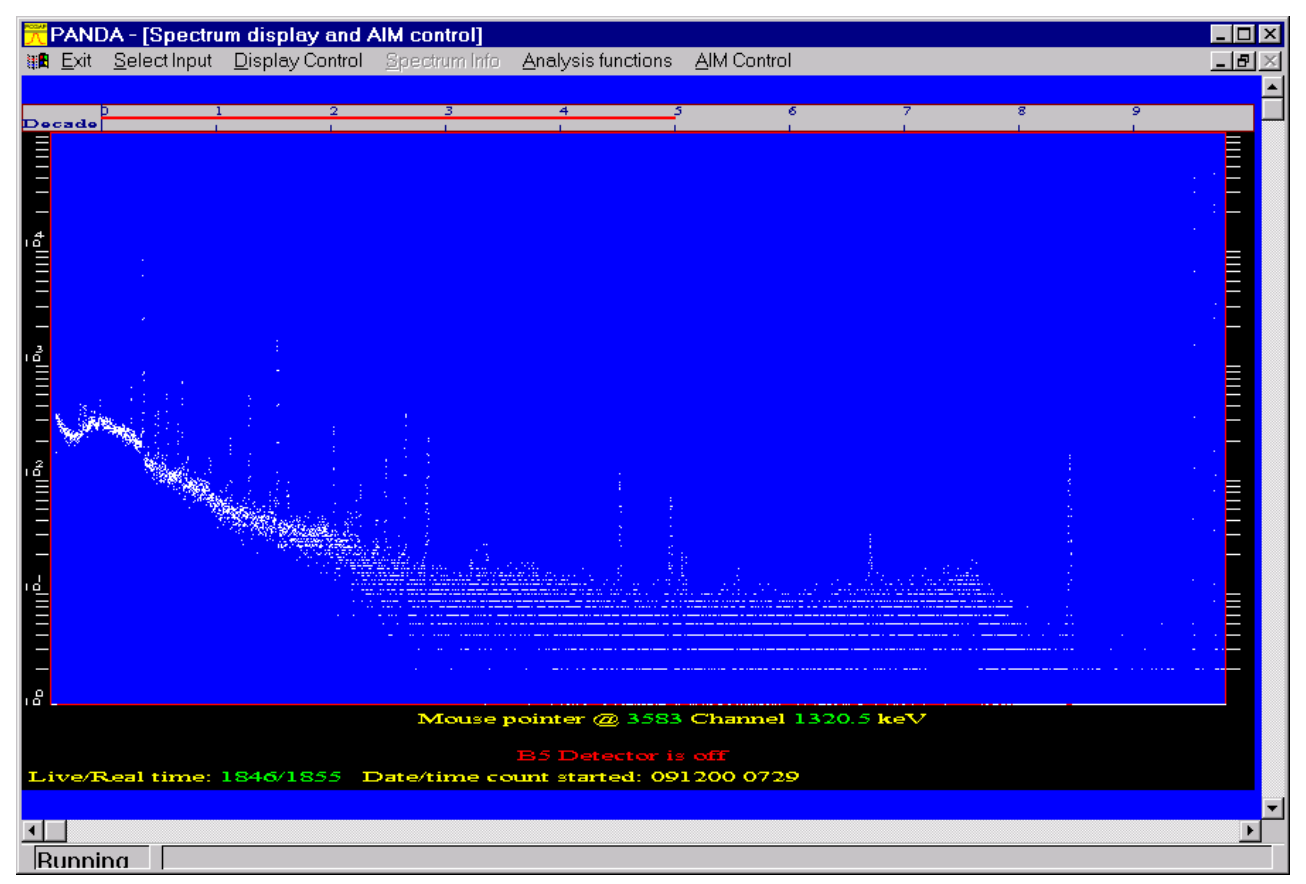

Figure E-4. Spectrum Display

\section{- $\quad$ Remote AIM}

The Genie 2000 software allows computers which are on the same network to use a set of detectors controlled by one Virtual Data Manager (VDM). Selection of this source of data begins by displaying the following Dialog Box:

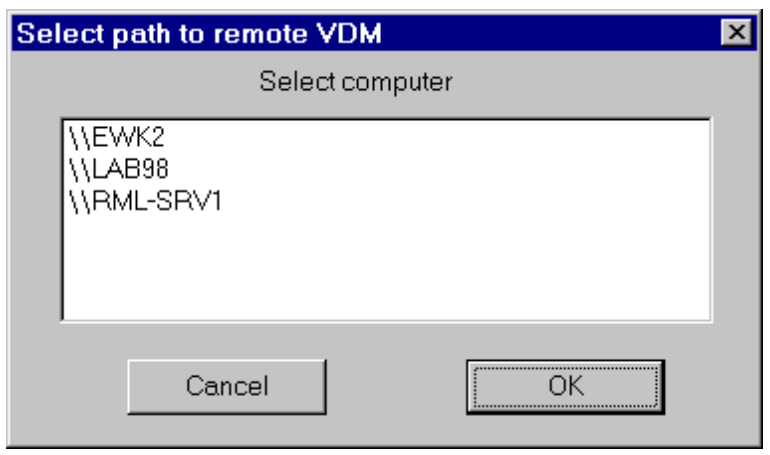

Figure E-5. Remote AIM beginning dialog

The user then selects the computer on which the controlling VDM is running. The computers displayed in this dialog box come from the contents of the G2K_VDM.NCF file located in Genie $2 k \mid C t l f i l e s$ directory. See Canberra's documentation for remote VDM access. Once the computer has been selected the selection of a detector system proceeds in the same manner as if the Local AIM data source was selected (See section "From Local AIM" above).

- From File

This data source option allows the user to select a spectrum file, which has been previously saved in PCGAP format. When this data source is selected an open file dialog box will be displayed. 


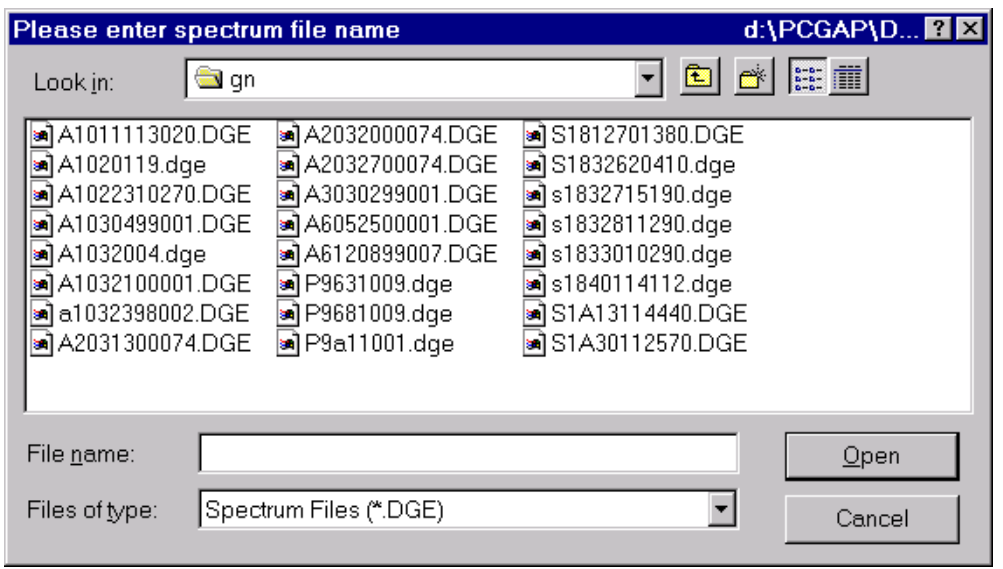

Figure E-6. Open file dialog

This open file dialog box operates in a standard Windows way. Most of the PCGAP standard files have ".DGE" extension. However, the slot files located in $\backslash$ PCGAP $\backslash D A T A \backslash S L O T \backslash$ have extension ".DAT" and can also be opened for display by PANDA. The extension of the file is not important as long as it is a standard PCGAP spectrum file. 


\section{E-3.0 AIM Control Menu}

Selection of an AIM as a data source (either Remote or Local) will generate an "AIM Control" pull down menu. This menu will list the following functions:

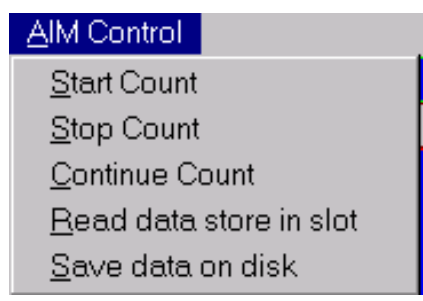

Figure E-7. AIM Control Functions

The "Start Count", "Stop Count" and "Continue Count" are self-explanatory functions. Following is an explanation of the other two functions.

- Read data store in slot

The "Read data store in slot" function reads the spectral data stored in the AIM and stores the data in a reusable disk file called a slot. The slot files are located in the $\backslash$ PCGAP\DATA $\backslash S L O T \backslash$ folder. The slot folder contains at least two files: a calibration file and a spectrum data file. These two files are called a slot pair. The name of slot pair files must be the same. The file names must be a twocharacter combination such as A1 or A2. The calibration file must have extension "CLB" and the spectrum file extension "DAT". Some examples of valid slot pairs are:

\section{A1.clb A1.dat These two files would create an A1 slot pair A2.clb A2.dat An A2 slot pair}

Slot pairs or Slots are used to temporarily store information, which can be accessed and used by different programs. The information stored in a slot pair is used to create a spectrum file using the PCGAP HEAD program. The information in the calibration file for a slot pair can be written only by one of the energy scale determination programs (See Section E-4.3 Analysis Function Menu). When one of these calibration programs is executed they determine the values for the coefficients of the energy and width equations. In case an INEEL pulser equipped multi-channel analyzer is used to collect spectral data, the automated calibration routines will also determine pulser equivalent energies and store these values in the calibration block. Once the calibration parameters have been determined and stored in the calibration file, subsequent transfers of spectral data from the analyzer will read the information stored in the calibration file to create a PCGAP spectrum file. To reiterate, once calibration parameters have been determined and stored in the calibration file of a slot pair, all spectrum file creations will inherit the information stored in the slot calibration file. This allows the operator to calibrate once and use those parameter values for all subsequent spectra collected by the system. The user can create as many slot pairs has he/she desires. Usual practice is to create a slot pair for every different detector system defined in the VDM. 
- Save Data On disk

This function reads the data out of the AIM and creates a PCGAP spectrum file in a save area in a subdirectory of the $\backslash$ PCGAP $\backslash$ DATA $\backslash S P E C T R A \backslash S A V E \backslash$ folder. The SAVE directory has one or more sub-folders. Spectrum files are saved in these sub-folders. These sub-folders denoted as "Save areas" are used to save spectral data from different types of samples. For example a "Save area" could be used to store all spectra collected from a particular waste stream or area. The initial PCGAP configuration contains two "Save areas"; GN and BK. The BK save area is used to save ambient background spectral files. GN is a general "Save area" where any type spectrum can be stored. The user can create additional "Save areas"; they must be sub-folders of the SAVE directory and have two character names. The spectrum analysis code expects a two-character name for the save area. Except for the BK save area, there is no restriction on which two characters can be used. The analysis code expects the ambient background spectra to be stored in the BK save area, consequently; this save area must exist.

Selection of this function generates the following dialog box:

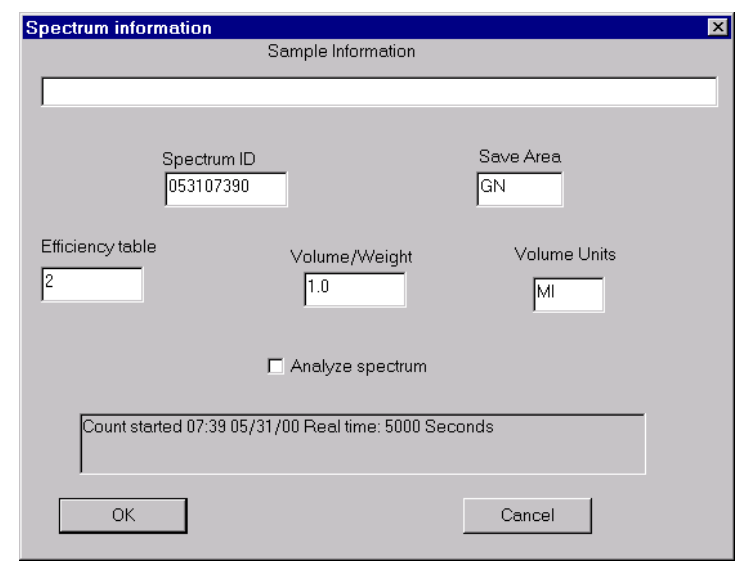

Figure E-8. Save data on disk dialog

Information describing the sample can be entered in the "Sample Information" text box. This information will be placed in the spectrum header. Information entered in the "Spectrum ID" text box is used to create the spectrum file name. The name will be constructed from the "two-character detector id" followed by the information entered in the Spectrum ID box and the ".dge" extension. The Save Area is the two-character save area (see proceeding paragraph). Finally the user must enter values for the efficiency table to use the sample volume/weight and units. An efficiency table of 2 has a value of 1.0 at all energies. Clicking the Analyze spectrum check box will cause the spectrum to be analyzed when $\mathrm{OK}$ is clicked. The information displayed in the bottom static box describes the date and time when the count was started. 


\section{E-4.0 Other PANDA Menus}

A spectrum display is generated (See figure E-4) after the user has selected the data source. The spectral display can be manipulated using the mouse. A left mouse click will "zoom" the display to a smaller number of channels. A right click in the spectral region of the display will generate a peak identification box:

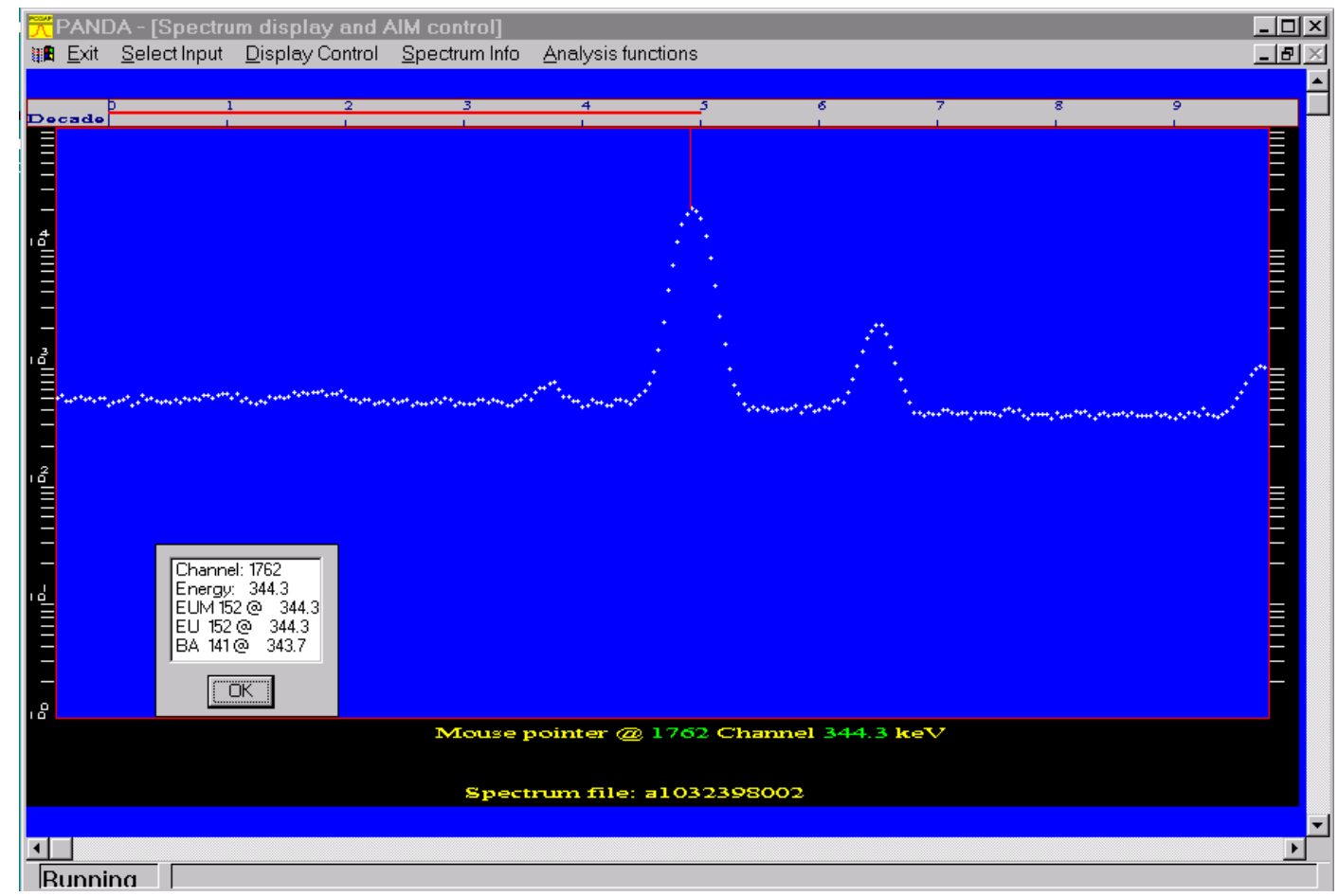

Figure E-9. Identification dialog

The identification box contains the channel and energy of the channel where the mouse cursor was pointing when the right click was made. Following the channel and energy values is a list of up to 3 radionuclides that have a gamma ray within $1 \mathrm{keV}$ of the channel energy. The identifications are made from the "MASTER.lib" nuclide library.

The display now has three new menu items: Display Control, Spectrum Info, and Analysis Functions. If the selected data source was an AIM the Spectrum Info menu will be grayed out i.e., the functions under the Spectrum Info menu item are not available unless the displayed data is from a file.

When the spectra data has been displayed a gray bar labeled "Decade" will be drawn with a red line running through it. For example:

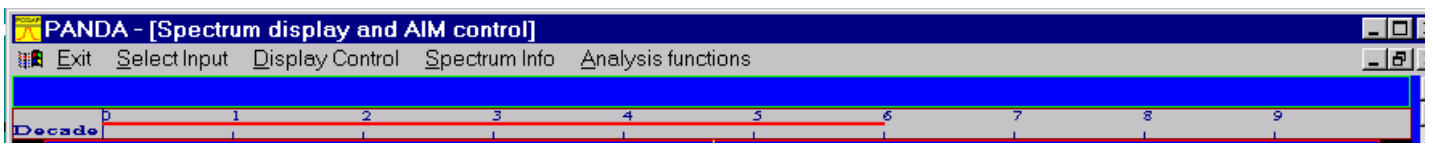

Figure E-10. Decade Selected

The gray bar is labeled with integer values 0 to 9. The "red line" describes the number of decades shown on the Y-axis. In the above example the Y-axis begins at $10^{0}$ and extends to $10^{6}$. The 
user can control the Y-axis scaling by clicking on the left side of the bar and the maximum number of decades by clicking to the right end of the bar.

At the bottom of the spectral display is a status area. This status area contains the following; the channel number of the mouse pointer and the energy scale value at that channel. Also, the spectrum file ID is displayed. See figure E-9.

\section{E-4.1 Display Control}

The following two functions are contained under this menu item:

Figure E-11. Display Control functions

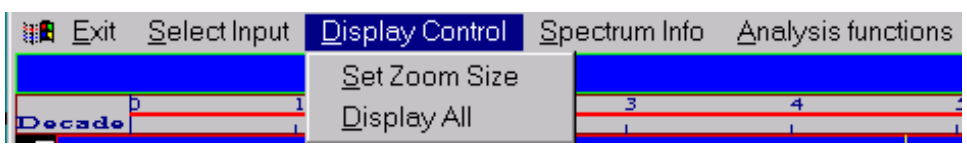

\section{- $\quad$ Set Zoom Size}

A click in the spectral display region will "zoom" the display to a region in the spectrum centered at the $\mathrm{x}$-axis where the click was made. The number of channels displayed when the display is zoomed is controlled by the "Set Zoom Size" function. A click on this function will generate the following dialog box:

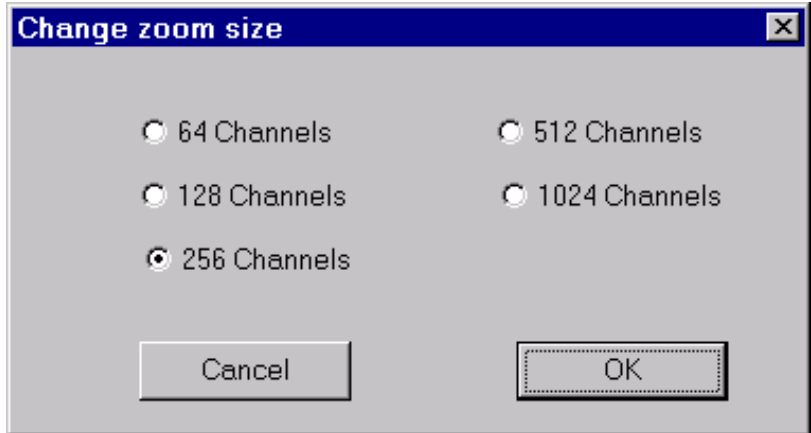

Figure E-12. Set Zoom size dialog box

Clicking on one of the radio buttons and clicking OK will set the number of channels displayed on a zoomed spectrum. In the following example the spectrum was zoomed to 256 channels. 


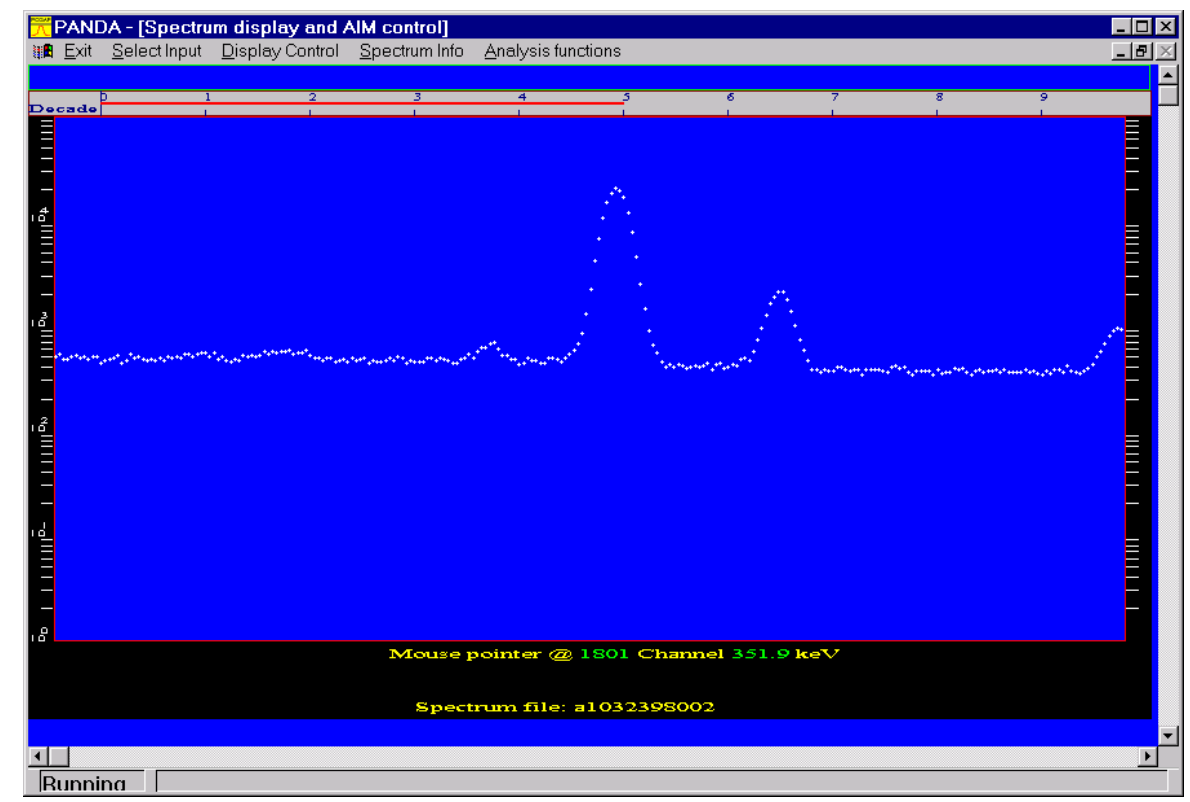

Figure E-13. Spectrum zoomed to 256 channels

- Display All

This function will "unzoom" a spectral display i.e. display all channels in the spectrum.

\section{E-4.2 Spectrum Info}

This menu item has the following functions:

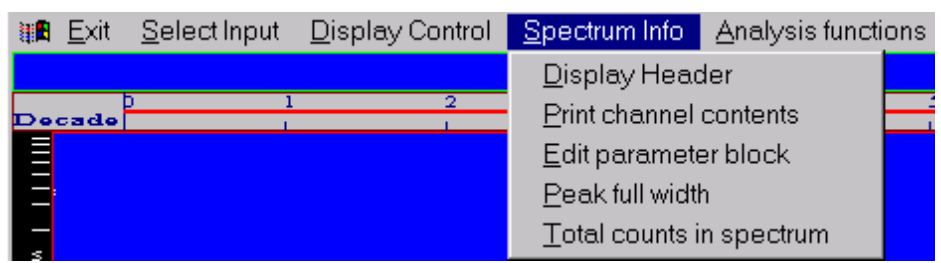

Figure E-14. Spectrum Info functions

Please note the Spectrum Info menu will only be active if the data source is a spectrum file, not any of the AIM data sources. The following sections will describe the functions.

- Display Header

Selection of this function will generate a dialog box, which contains the more common spectral header and parameter values. For example: 


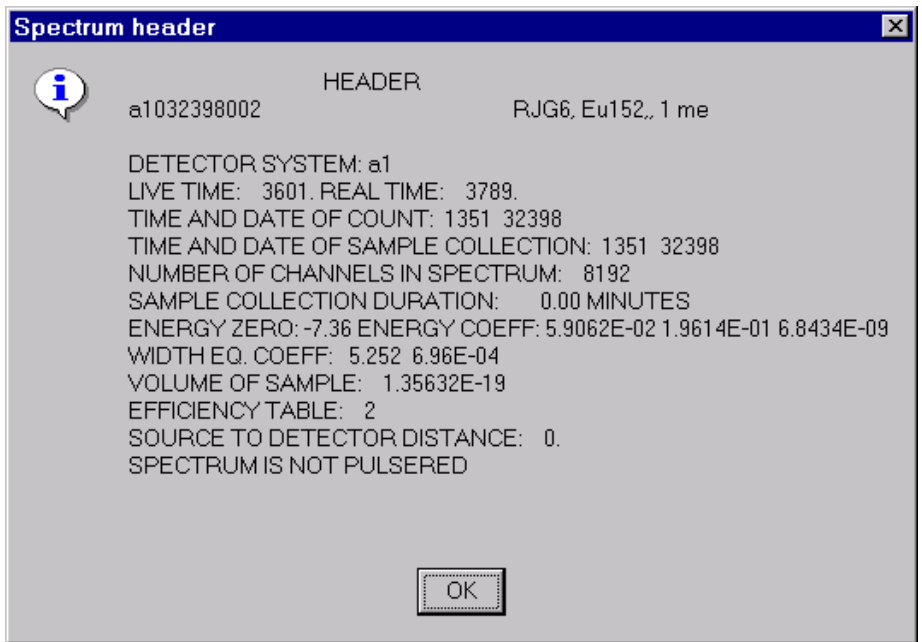

Figure E-15. Display of spectrum information

- Print Channel Contents

Selecting this function generates the following dialog:

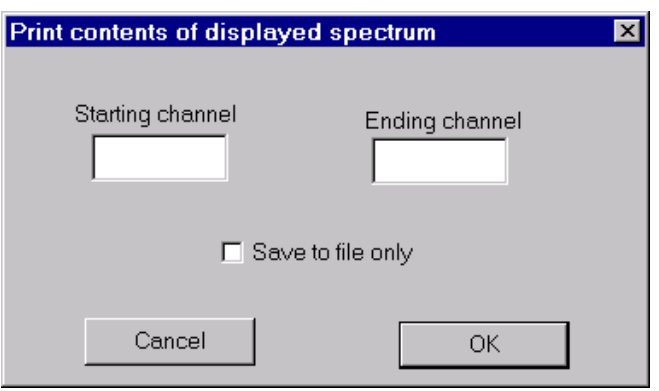

Figure E-16. Print Channel Contents dialog box

The user must enter a starting channel number and an ending channel number. The contents of the channels between the starting and ending channels will be printed on the default printer or if the "Save to file only" check box is selected an ASCII coded file will be created in $\backslash$ PCGAP\PRDATA.TXT.

- Edit Parameter Block

Each spectrum has a 512 byte record which contains various parameters. These values for these parameters can be modified using this function. The parameters that can be modified include:

- Efficiency table number

- Number of injected pulser peaks

- Low pulser equivalent energy value

- High pulser equivalent energy value

- Low Pulser offset

- High pulser offset

- Low pulser buffer start channel

- High pulser buffer start channel 
- Energy zero offset (in channels)

- Energy equation (Energy $\left.=\mathrm{A}+\mathrm{Bx}+\mathrm{Qx}{ }^{2}\right)$, “A" coefficient

- Energy equation $\left(\right.$ Energy $\left.=\mathrm{A}+\mathrm{Bx}+\mathrm{Qx}^{2}\right)$, "B" coefficient

- Energy equation (Energy $\left.=\mathrm{A}+\mathrm{Bx}+\mathrm{Qx}{ }^{2}\right)$, “Q" coefficient

- Width equation (Width $=\mathrm{A}+\mathrm{Bx}$ ) "A" coefficient

- Width equation (Width $=\mathrm{A}+\mathrm{Bx}$ ) "B" coefficient

- Random summing correction equation ( Correction $=\mathrm{A}+\mathrm{Bx}+\mathrm{Cx}^{2}$ ) " $\mathrm{A}$ " coefficient

- Random summing correction equation ( Correction $\left.=\mathrm{A}+\mathrm{Bx}+\mathrm{Cx}^{2}\right)$ " $\mathrm{B}$ " coefficient

- Random summing correction equation ( Correction $=\mathrm{A}+\mathrm{Bx}+\mathrm{Cx}^{2}$ ) "C" coefficient

- Width of pulser peak when calibrated

- Volume of sample

- Volume units

- Sample collection time (HHMM)

- Sample collection date (MMDDYY)

- Sample collection duration in hours

- Detector used to collect spectrum

- $\quad$ Start count date (MMDDYY)

- Start count time (HHMM)

The Edit Parameter Block function begins execution by displaying the following dialog:

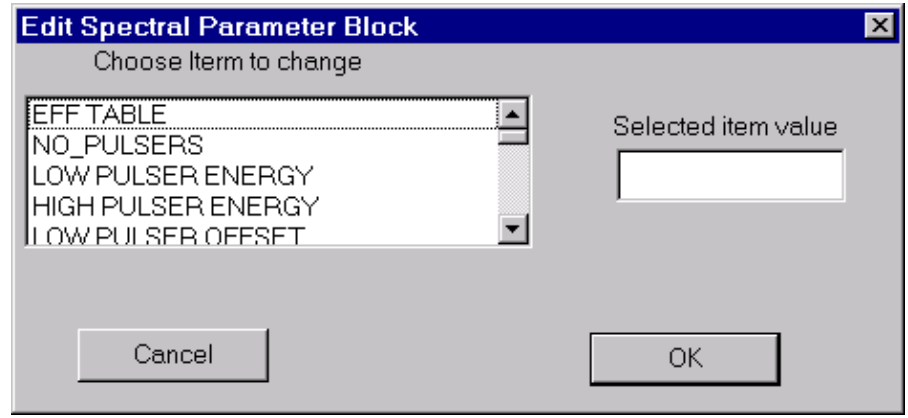

Figure E-17. Edit Parameter Dialog box

To modify one of the parameter values click on the parameter name on the left list box; its value will be shown in the edit box on the right. Modify the value in the edit box and click OK; the spectrum parameter block will now be updated with the new value.

- Peak Full Width

This function computes the full width at half max, the full width at $10^{\text {th }}$ max and the full width at $50^{\text {th }}$ max and displays the values. The values are computed from the spectral channel contents. To use the function, position the mouse pointer on the photopeak and left click. A black line will then be drawn to the top of the display. A right click will then display the peak parameters. Click on "exit" to exit this function. .

- Total Counts in the Spectrum 
This function computes the total counts in the spectrum and displays the value. In spectra equipped with INEEL pulser, the total counts don't include the pulser data.

\section{E-4.3 Analysis Functions Menu}

Clicking on this menu item generates the display of the following functions:

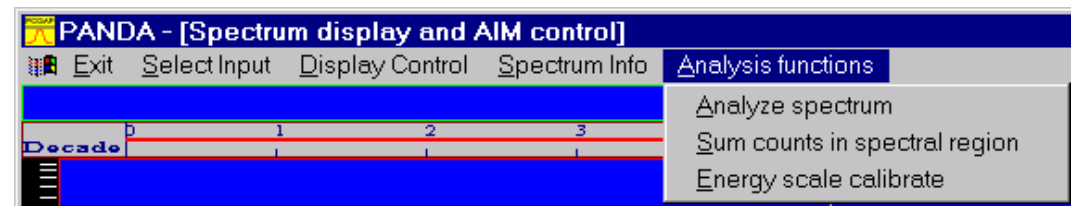

Figure E-18. Analysis Functions

Each of these functions is described in the following sections.

- Analyze Spectrum

This function is used to perform an automatic spectrum analysis. The following dialog is displayed when this function is selected:

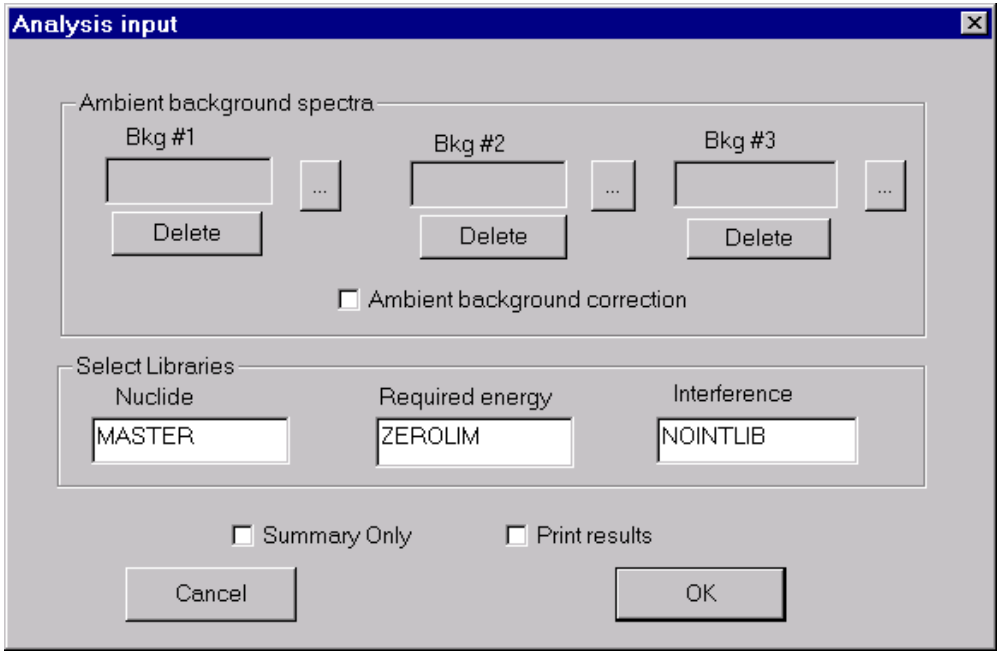

Figure E-19. PANDA Analysis Dialog.

This dialog box has two sections; the ambient background section and the libraries section.

Up to three background spectra can be averaged to determine the ambient background contribution for each photopeak analyzed in the sample spectrum. Associated with each background edit box (Bkg \#1, Bkg \#2, Bkg \#3) is a browse button. The spectrum ID can be typed directly into the box or the associated browse button can be used to locate the desired background spectrum. The background must be located in the BK subdirectory, PCGAP\DATA ISPECTRA ISAVE $\backslash B K$. The spectrum for Bkg \#1 must be chosen first, then Bkg \#2 and finally Bkg \#3. 
The names of the Nuclide, Required energy and Interference libraries must be typed in the edit box for each type of library. The ZEROLIM library shown in the example has no entries in it and no directed analysis will be performed. These library files must be located in their associated subdirectories:

\section{Nuclide... PCGAP $\backslash$ DATA $\backslash L I B R A R Y \backslash N U C L I D E$. Required energy... PCGAP\DATA $\backslash L I B R A R Y \backslash R E Q U I R E D$. Interference... PCGAP $\backslash$ DATA $\backslash L I B R A R Y \backslash I N T E R$}

Finally, the user can have the results of the analysis printed on the default printer. Either the full analysis output can be printed which includes a photopeak by photopeak analysis description, and a nuclide line by line analysis description section. Or a nuclide summary section can be printed where the net concentration value for each nuclide is printed.

After the spectrum analysis is completed, the full analysis output can always be found in PCGAP $\backslash$ CBAG_OUT.LIS. This file contains the results of the most recent analysis and can be viewed using Note Pad or any other text editor. To get a hard copy output of this file use the PCGAP program "Print File". This program will use a font and paper orientation that will result in the proper output.

- Sum counts in spectral region

This function is used to compute the sum of the counts in a region on the spectrum. Both a gross and net sum are computed. The net sum is the sum of the counts above a background line drawn between the left and right region boundaries. When this function is selected the following display will be generated. In this example the spectrum display was zoomed before the Sum function was initiated.

Figure E-20. Sum region function

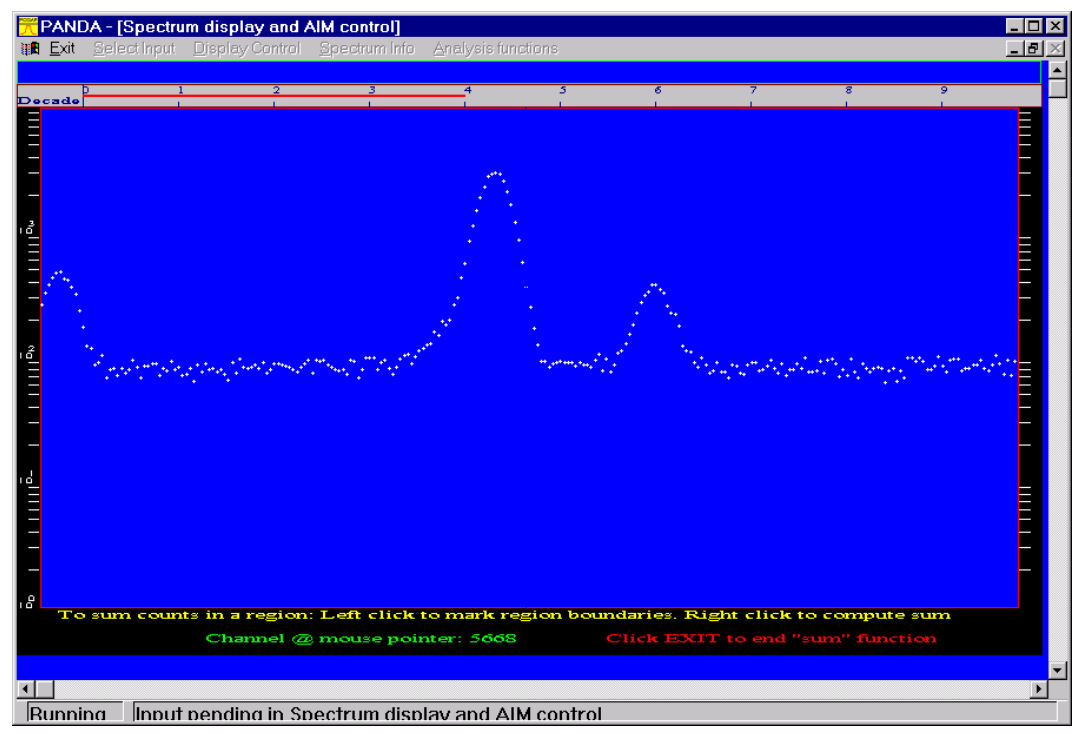

To select a region to sum use the left mouse button. Position the mouse pointer on one boundary and left click. Then move to the other region boundary and left click again. A solid black line will be drawn where the left clicks are made. The spectrum channel of the mouse pointer is displayed in the status area of the display. The information will aid in positioning of the mouse 
pointer at a particular channel. Once the boundaries of the region have been defined a right click will compute the sums. For example:

Figure E-21. Region data

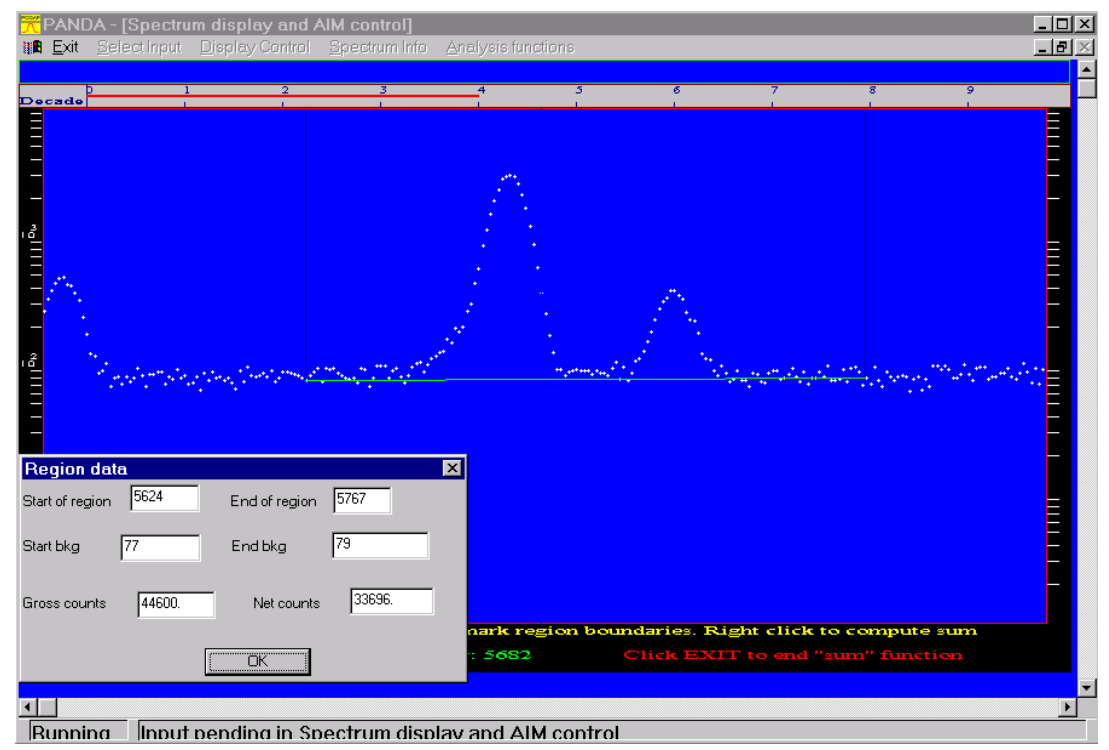

A background line is drawn between the two end points of the region. The counts in the channels at the boundary are displayed in the "Region data" dialog box as "Start bkg" and "End bkg" values respectively. They are used to determine the background line. The "Net counts" value is the sum of all counts above the background line. The "Gross counts" value is the sum of the counts in the channels between and including the region boundary. Clicking the OK function box will dismiss the dialog box and another sum computation can be preformed. To return to the normal PANDA display click on the "Exit" menu.

\section{- Energy scale Calibrate}

PANDA has the ability to determine the energy scale of a spectrum using three different methods:

1. An automated method using a $\mathrm{Th}^{228}$ calibration source. Used for energy scales in the range of 0 to $3 \mathrm{MeV}$.

2. An automated method using an $\mathrm{Eu}^{152}$ calibration source. Used for energy scales nominally in the range of 0 to $2 \mathrm{MeV}$.

3. A manual method where the user identifies peaks in the spectrum and supplies their energies and peak widths.

The energy scale in PCGAP is expressed as a quadratic equation of the form

$$
\mathrm{E}(\mathrm{x})=\mathrm{A}+\mathrm{B}(\mathrm{x})+\mathrm{C}\left(\mathrm{x}^{2}\right) \text { where } \mathrm{x} \text { is a channel number. }
$$

A photopeak width equation, $\mathrm{W}(\mathrm{x})$, is used in PCGAP to determine the initial width value for the peak fitting processes and is a linear function of the form:

$$
\mathrm{W}(\mathrm{x})=\mathrm{F}+\mathrm{G}(\mathrm{x}) \text { where } \mathrm{x} \text { is a channel number. }
$$


Determination of Energy equation parameters (A, B, and C) and the width parameters (F and G) can be performed by energy scale determination programs.

The automatic programs operate basically in the same manner. They use a calibration spectrum with a known photopeak structure to find the location of five photopeaks of known energy in the spectrum, and then perform a non-linear least squares fit to each, to determine their widths. A linear least squares fit is then made to a $2^{\text {nd }}$ order polynomial (photopeak centroid versus energy) to determine the three energy equation parameters. Likewise, a linear least squares fit is made to $1^{\text {st }}$ order polynomial (photopeak centroid versus width) to determine the width equation parameters. The following table describe the photopeaks used in these algorithms.

\begin{tabular}{cc}
\hline & \\
\hline $\mathrm{Th}^{228}$ photopeak energies $(\mathrm{keV})$ & $\mathrm{Eu}^{152}$ photopeak energies \\
& $(\mathrm{keV})$ \\
\hline 238.632 & 121.783 \\
583.191 & 344.274 \\
860.564 & 778.872 \\
1620.735 & 1112.092 \\
2614.533 & 1408.024 \\
\hline
\end{tabular}

The automatic calibration routines begin by finding the photopeak with the largest amplitude in the last $1 / 2$ of the spectrum (channels 4100 to 8000 ). That photopeak is assigned the largest energy. For $\mathrm{Th}^{228}$ the assigned peak energy is 2614.533, for $\mathrm{Eu}^{152} 1408.024$ is used. By knowing the channel and energy of the large energy photopeak an estimate can be made as to the location of the smallest energy photopeak. The zero of the spectrometer must be adjusted so that the anticipated channel of the small energy photopeak is within 50 channels of its calculated value. Once the smallest and largest energy photopeaks have been determined, the locations of the other three photopeaks can be determined from a linear line extrapolated between the two photopeak positions and energies.

The energy scale program not only determines the energy scale of the current spectrum, but also determines the energy scale of the slot file for the associated detector (See section "Read data store in slot"). These calibration values can then transferred to other spectra as they are read from a multichannel analyzer or converted from another spectral format (See PCGAP program CONVERT).

The energy scale determination function begins by displaying the following dialog box:

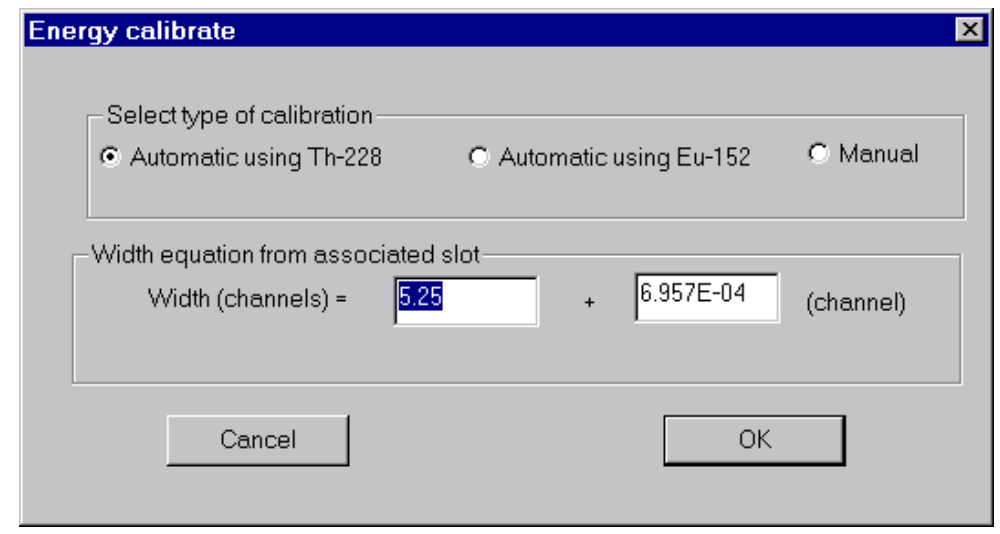

Figure E-22. Energy scale determination Dialog box 
The user uses the radio buttons to select the type of analysis to use in determining the energy scale. The current width equation parameter values for the associated slot file are also displayed. The user can edit these parameter values, in the case the detector system being calibrated has drastically different values for these parameters. In the automatic energy scale determination mode the width equation is used to determine the fit limits for the five peaks being fit. If the parameter values for the width equation does not approximately match the data, the fit will fail.

For the manual energy scale calibration, the user must enter the centroids and associated energy for at least two photopeaks in the spectrum. The program will then fit the photopeaks at the specified locations to refine the centroid value and determine the full width at half max. In the case where values were entered for at least three photopeaks, a linear least squares fit is made to a $2^{\text {nd }}$ order polynomial (photopeak centroid versus energy) to determine the three energy equation parameters. Likewise, a linear least squares fit is made to $1^{\text {st }}$ order polynomial (photopeak centroid versus width) to determine the width equation parameters. If only values for two photopeaks were entered then the least squares fit is made to $1^{\text {st }}$ order polynomial to determine the parameters of the energy equation.

At the successful conclusion of an energy scale determination the user will be asked if the calculated energy scale parameter values should be used to update the values for the corresponding slot file. In normal situations this should be answered in the affirmative; otherwise only the current spectrum will be updated with the new energy scale. 